\title{
AN H I SURVEY OF SIX LOCAL GROUP ANALOGS. II. H I PROPERTIES OF GROUP GALAXIES
}

\author{
D. J. Pisano ${ }^{1,7}$, David G. Barnes ${ }^{2}$, Lister Staveley-Smith ${ }^{3}$, Brad K. Gibson ${ }^{4,5}$, \\ Virginia A. KILBORN ${ }^{2}$, AND Ken C. FreEman ${ }^{6}$ \\ ${ }^{1}$ Department of Physics, West Virginia University, P.O. Box 6315, Morgantown, WV 26506, USA; djpisano@mail.wvu.edu \\ ${ }^{2}$ Centre for Astrophysics and Supercomputing, Swinburne University, Hawthorn, Victoria 3122, Australia; David.G.Barnes@gmail.com, \\ vkilborn@astro.swin.edu.au \\ ${ }^{3}$ International Centre for Radio Astronomy Research, M468, University of Western Australia, Crawley, WA 6009, Australia; Lister.Staveley-Smith@ icrar.org \\ ${ }^{4}$ Jeremiah Horrocks Institute, University of Central Lancashire, Preston PR1 2HE, UK; brad.k.gibson@ gmail.com \\ ${ }^{5}$ Department of Astronomy and Physics, Saint Mary's University, Halifax, Nova Scotia B3H 3C3, Canada \\ ${ }^{6}$ RSAA, Mount Stromlo Observatory, Cotter Road, Weston, ACT 2611, Australia; kcf@mso.anu.edu.au \\ Received 2011 September 2; accepted 2011 October 13; published 2011 November 29
}

\begin{abstract}
We have conducted an H I $21 \mathrm{~cm}$ emission-line survey of six loose groups of galaxies chosen to be analogs to the Local Group. The survey was conducted using the Parkes multibeam instrument and the Australia Telescope Compact Array (ATCA) over a $\sim 1 \mathrm{Mpc}^{2}$ area and covering the full depth of each group, with an $M_{\mathrm{H}}$ sensitivity of $\sim 7 \times 10^{5} M_{\odot}$. Our survey detected 110 sources, 61 of which are associated with the six groups. All of these sources were confirmed with ATCA observations or were previously cataloged by HIPASS. The sources all have optical counterparts and properties consistent with dwarf irregular or late-type spiral galaxies. We present here the $\mathrm{H}$ I properties of the groups and their galaxies. We derive an H I mass function (HIMF) for the groups that is consistent with being flatter than the equivalent field HIMF. We also derive a circular velocity distribution function, tracing the luminous dark matter halos in the groups, that is consistent with those of the Local Group and HIPASS galaxies, both of which are shallower than that of clusters or predictions from cold dark matter models of galaxy formation.
\end{abstract}

Key words: galaxies: evolution - galaxies: formation - galaxies: groups: general - galaxies: luminosity function, mass function - Local Group

Online-only material: color figures

\section{INTRODUCTION}

The majority of galaxies, including the Milky Way, reside in groups (Geller \& Huchra 1983; Tully 1987; Eke et al. 2004; Tago et al. 2008), as such it is essential to study these structures if we wish to understand the effect of the environment on galaxy properties. A galaxy group is a very broad classification that has not been very precisely defined in the literature and whose properties span a wide range of mass and density (amongst others). They range in size from massive, rich groups to lowmass poor, loose groups and compact groups. The rich groups tend to be dominated by early-type galaxies (Postman \& Geller 1984; Helsdon \& Ponman 2003) and have an X-ray bright, intra-group medium (IGM; Mulchaey 2000; Mulchaey et al. 2003) that may result in ram pressure stripping of gas-rich spiral galaxies (Sengupta \& Balasubramanyam 2006; Sengupta et al. 2007). In these ways, rich groups are very similar to low-mass galaxy clusters. Compact groups are the densest groups with a range of masses, containing a few to tens of galaxies typically separated by only a couple of galaxy radii (e.g., Hickson 1982, 1997). These groups contain galaxies that are strongly interacting and can also host an X-ray bright IGM (Ponman et al. 1996) potentially generated by the tidal interactions of the group members (Verdes-Montenegro et al. 2001).

In contrast to both of these classes, loose, poor groups are similar to the Local Group. They are less massive than rich groups, although with similar numbers of member galaxies.

\footnotetext{
7 Adjunct Assistant Astronomer at National Radio Astronomy Observatory, P.O. Box 2, Green Bank, WV 24944, USA.
}

They can be dominated by either late-type or early-type galaxies, but only the groups containing at least one early-type galaxies have a hot, X-ray emitting IGM (Mulchaey 2000; Mulchaey et al. 2003). This suggests that ram pressure stripping is unlikely to have a large effect on galaxies in most of these groups (cf. Grcevich \& Putman 2009). These groups are diffuse with low velocity dispersions resulting in crossing times that are comparable to a Hubble time and, as such, they are unlikely to be virialized (Zabludoff \& Mulchaey 1998). This implies that interactions are rare, but, due to the low velocity dispersions, are more effective than in clusters and rich groups. These interactions may even strip $\mathrm{H}_{\mathrm{I}}$ from galaxies in loose, poor groups (Omar \& Dwarakanath 2005).

While there have been a number of studies of the gaseous properties of isolated galaxies (Haynes \& Giovanelli 1984; Pisano \& Wilcots 1999; Pisano et al. 2002), of cluster galaxies (e.g., Bravo-Alfaro et al. 2000, 2001; Chung et al. 2009), and of compact and rich groups (e.g., Williams \& Rood 1987; Williams et al. 1991; Verdes-Montenegro et al. 2001; Freeland et al. 2009; Kilborn et al. 2009; Borthakur et al. 2010), there have been fewer targeted studies of poor, loose groups analogous to our own Local Group. This paper seeks to fill that gap by exploring the neutral hydrogen properties of galaxies in six nearby, loose groups.

Mass functions serve as an excellent test of models of galaxy formation and are a simple of way quantifying differences between galaxy populations in different environments and comparing observations with models (Snaith et al. 2011). Current models of cold dark matter (CDM) galaxy formation predict an excess of low mass, as parameterized by their circular 

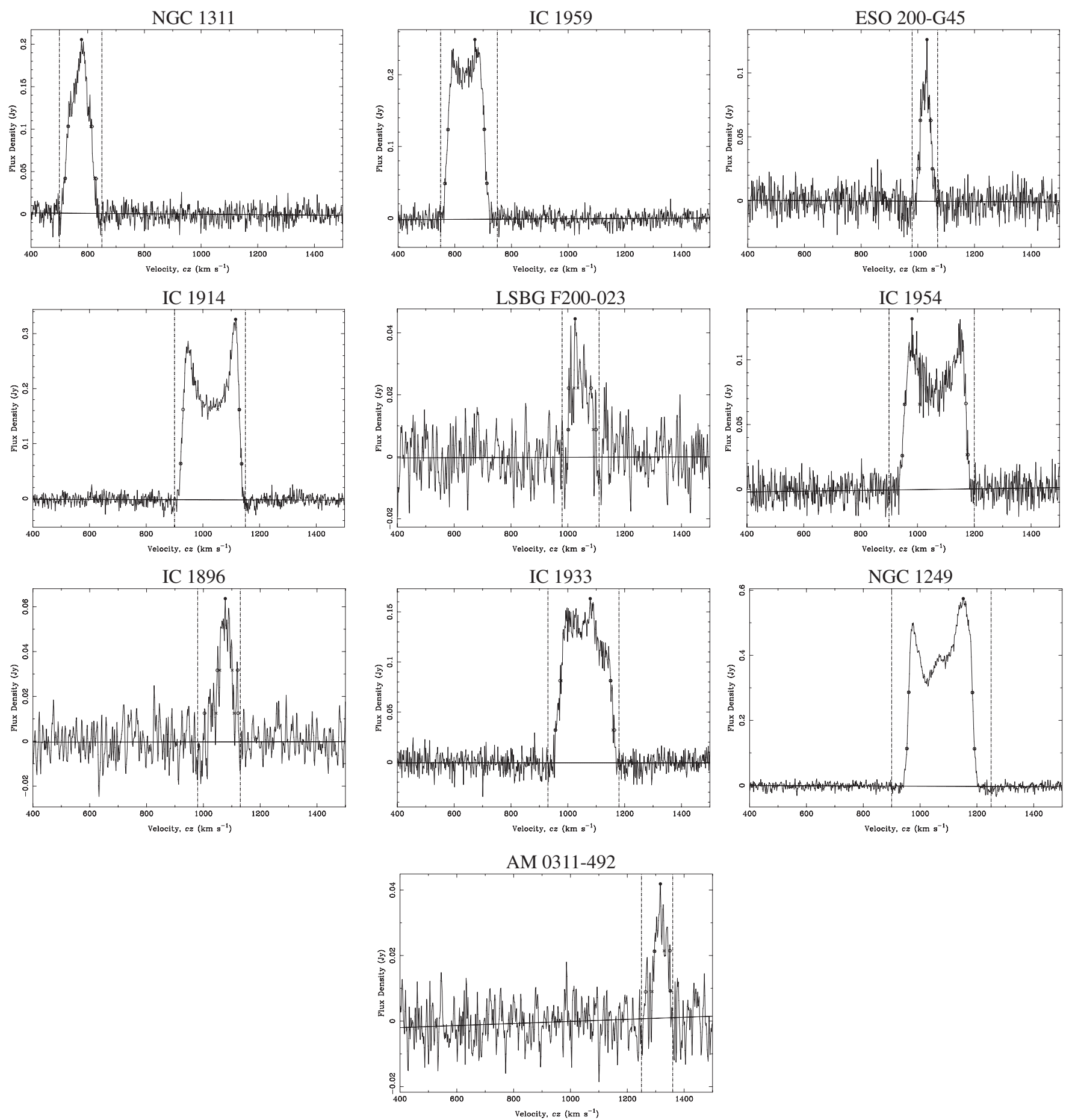

Figure 1. Parkes H I spectra of the confirmed detections in LGG 93. The vertical dashed lines indicate the range of velocities over which the profile properties were measured. The filled circles indicate the peak of the profile; the open circles the maximum $20 \%$ and $50 \%$ velocity widths; the $\times$ 's mark the minimum $20 \%$ and $50 \%$ widths. The nearly horizontal solid line indicates the baseline fit to the spectrum.

velocity (the circular velocity distribution function, CVDF), dark matter halos as compared to what is observed locally (Klypin et al. 1999; Moore et al. 1999). While some authors have directly measured the CVDF for luminous galaxies (e.g., Shimasaku 1993; Sheth et al. 2003; Goldberg et al. 2005), this is difficult to do for very low mass dwarf galaxies; Blanton et al. (2008) and others have used different methods to infer the CVDF or used alternative proxies. The optical luminosity function has been regularly used as a proxy (e.g., Tully et al. 2002; Trentham et al. 2005) for the CVDF. Luminosity functions can also be used in concert with the Tully-Fisher relation or fundamental plane to infer the CVDF (Cole \& Kaiser 1989; Gonzalez et al. 2000; Sheth et al. 2003; Desai et al. 2004; Goldberg et al. 2005). Unlike optical luminosity functions, using radio observations of $21 \mathrm{~cm}$ emission from neutral hydrogen $(\mathrm{HI})$ provides two measures of the mass of a galaxy: the integrated line profile yields the Hi mass while the linewidth provides the circular rotation velocity of the galaxy. Regardless of how the halo mass function is measured, be it by optical luminosity (Tully et al. 2002; Trentham et al. 2005), Hi mass (Zwaan et al. 

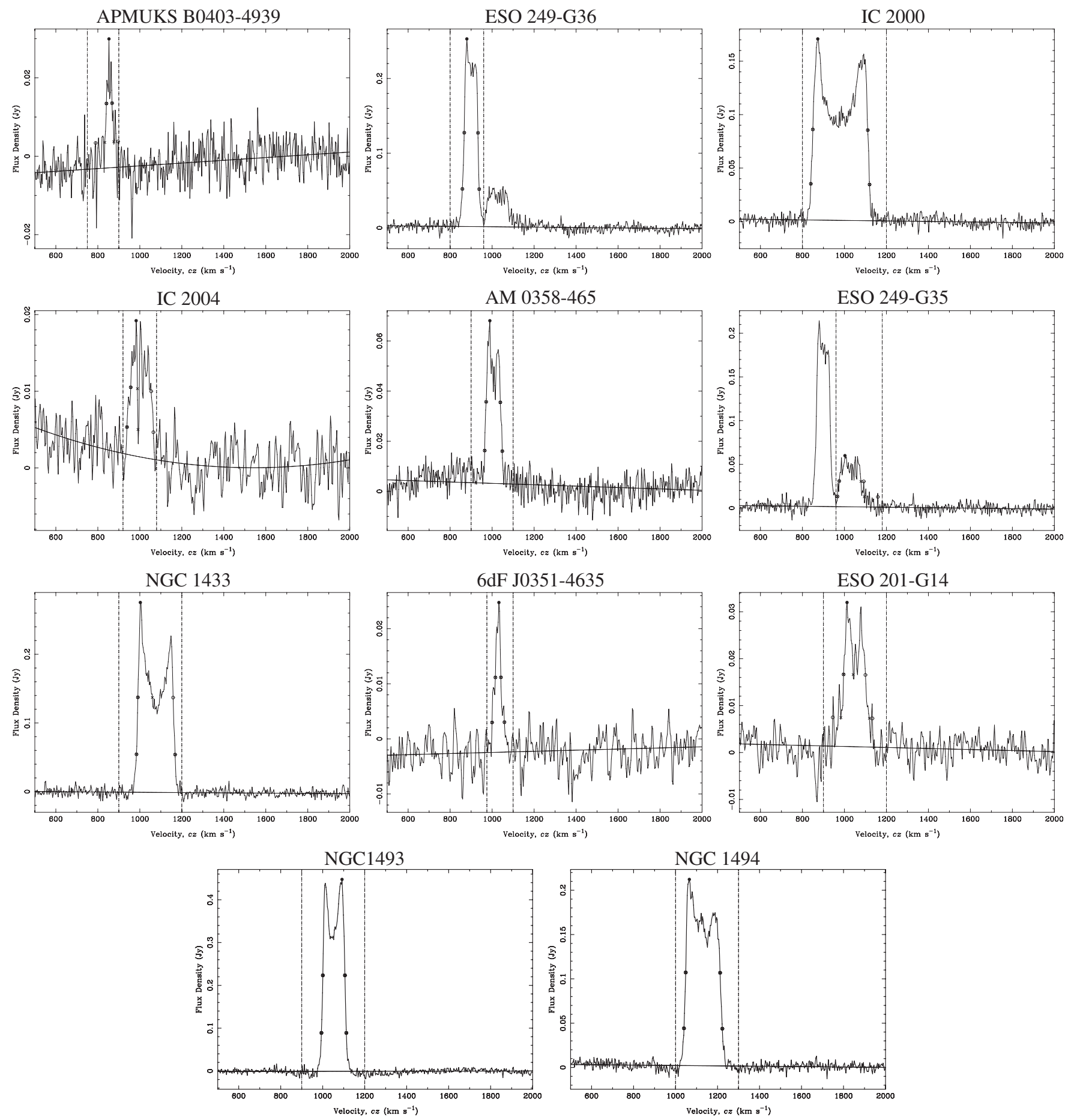

Figure 2. Same as Figure 1, but for LGG 106 group galaxies.

2005), or circular velocity (Zwaan et al. 2010), there is always a deficit of observed galaxies at low masses; this is the "missing satellite" or "substructure" problem (Klypin et al. 1999; Moore et al. 1999). Furthermore, there have been relatively few studies of how the Hi mass function (HIMF) and CVDF may vary with environment. In this paper, we will compare the HIMF and CVDF for our six loose groups with those of the galaxy population in general and in other specific environments.

This is the second of two papers concerning our survey. In the first paper (Pisano et al. 2007, hereafter Paper I), we described our selection criteria, survey parameters, observa- tions, data reduction, and the survey goals. These will be briefly summarized in this paper. We have already used our data to place constraints on the amount of intra-group $\mathrm{H}$ I clouds that may be analogous to the high-velocity clouds (HVCs; Wakker \& van Woerden 1997) seen around the Milky Way. Namely, that any such HVC analogs must reside within $90 \mathrm{kpc}$ of galaxies and have a total $M_{\mathrm{HI}} \lesssim 10^{8} M_{\odot}$ (Pisano et al. 2004; Paper I). In this paper, we will discuss the reliability of our survey and data analysis, present our Hi data on the galaxies in the six groups, derive an HIMF and CVDF for the loose group environment, and discuss the implications for the effect of environment 

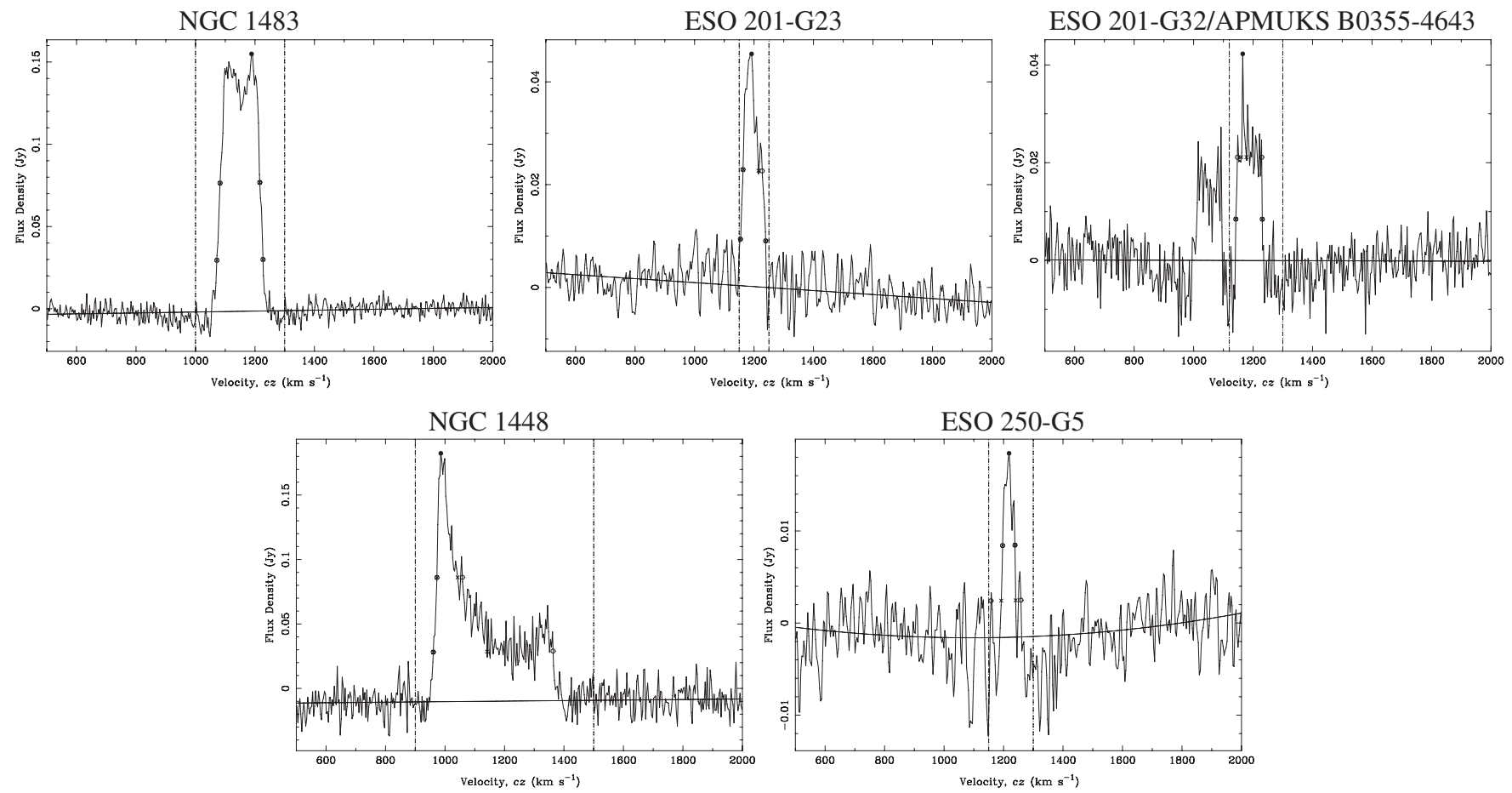

Figure 3. Same as Figure 1, but for the remaining LGG 106 group galaxies.

on galaxy formation. We summarize the sample selection in Section 2 and the observations and data reduction in Section 3. The results are presented in Section 4 including a description of the reliability and completeness of the survey, how we measure the galaxy properties, and a summary of the group and galaxy properties. Finally, we present the HIMF in Section 5, the CVDF in Section 6, and our conclusions in Section 7.

\section{SAMPLE SELECTION}

For this project, we identified six poor, loose groups of galaxies that are analogous to the Local Group. Details are given in Paper I, but the selection is summarized here. Groups were selected to be nearby $\left(v_{\mathrm{GSR}}<1000 \mathrm{~km} \mathrm{~s}^{-1}\right)$, but not confused with Galactic H I emission $\left(v_{\mathrm{GSR}}>300 \mathrm{~km} \mathrm{~s}^{-1}\right)$. The groups only contain spiral or irregular galaxies separated, on average, by a few hundred kiloparsecs with a total extent of $\sim 1 \mathrm{Mpc}$. Because our observations were made with the Parkes radio telescope in Australia, we only chose groups below a declination of $0^{\circ}$. We selected five groups, LGG 93, LGG 106, LGG 180, LGG 293, and LGG 478, from the Lyon Groups of Galaxies (LGG) catalog of Garcia (1993) and a sixth group from the HICAT group catalog of Stevens (2005). Distances are corrected using the multi-attractor velocity flow model of Masters (2005; K.L. Masters 2010, private communication), and assuming $H_{0}=72 \mathrm{~km} \mathrm{~s}^{-1} \mathrm{Mpc}^{-1}$ (Spergel et al. 2003). The measured properties of the groups are discussed in detail in Section 4.3.

\section{OBSERVATIONS AND DATA REDUCTION}

We observed the entire extent of the six groups between 2001 October and 2003 June using the $20 \mathrm{~cm}$ multibeam instrument (Staveley-Smith et al. 1996) on the Parkes $64 \mathrm{~m}$ radio telescope. ${ }^{8}$ Observations of the first two groups, LGG 93 and LGG 180, were made with an $8 \mathrm{MHz}$ bandwidth and $1.65 \mathrm{~km} \mathrm{~s}^{-1}$ channels using the inner seven beams of the multibeam, while all subsequent observations were made using all 13 beams, a $16 \mathrm{MHz}$ bandwidth, and $3.3 \mathrm{~km} \mathrm{~s}^{-1}$ channels. All groups were observed only at night to avoid solar interference. Maps were made by scanning in a basket-weave pattern in right ascension and declination with consecutive scans being offset to result in uniform coverage perpendicular to the scan direction. Data were calibrated using periodic observations of flux calibrator Hydra A with a resulting accuracy of about $10 \%$. All data were reduced and gridded using the LIVEDATA and GRIDZILLA packages. The $1 \sigma, 3.3 \mathrm{~km} \mathrm{~s}^{-1} \mathrm{rms}$ noise in these cubes range from 5.5 to $7.0 \mathrm{mJy}$, corresponding to an $M_{\mathrm{HI}}$ of $(3.5-11) \times 10^{5} M_{\odot}$ and $N_{H_{\text {I }}}$ of $(2.8-4.6) \times 10^{16} \mathrm{~cm}^{-2}$ depending on the group. For a $5 \sigma$ detection of a source with a $30 \mathrm{~km} \mathrm{~s}^{-1}$ linewidth, we have an $M_{\mathrm{HI}}$ limit of $(0.5-2) \times 10^{7} M_{\odot}$, and an $N_{H_{\mathrm{I}}}$ limit of $(4.2-6.9) \times$ $10^{17} \mathrm{~cm}^{-2}$. It is worth noting that our reduction technique will subtract out sources that are larger than a few beams across.

The final cubes were searched by three groups of authors: D.J.P., D.G.B., and B.K.G. and V.A.K. in tandem. Our final list of putative sources included those that were identified by at least two of the three groups of authors. In addition, our identification of fake sources added to our cubes by M. Zwaan allowed us to assess the completeness of our survey, as discussed in Paper I.

We used the Australia Telescope Compact Array (ATCA) ${ }^{9}$ to confirm the reality of the sources identified in our Parkes data. We observed 105 of the 112 Parkes detections in and behind the six groups in our sample. The remaining seven

\footnotetext{
8 The Parkes radio telescope is part of the Australia Telescope National Facility which is funded by the Commonwealth of Australia for operation as a National Facility managed by CSIRO.

9 The Australia Telescope is part of the Australia Telescope National Facility which is funded by the Commonwealth of Australia for operation as a National Facility managed by CSIRO.
} 

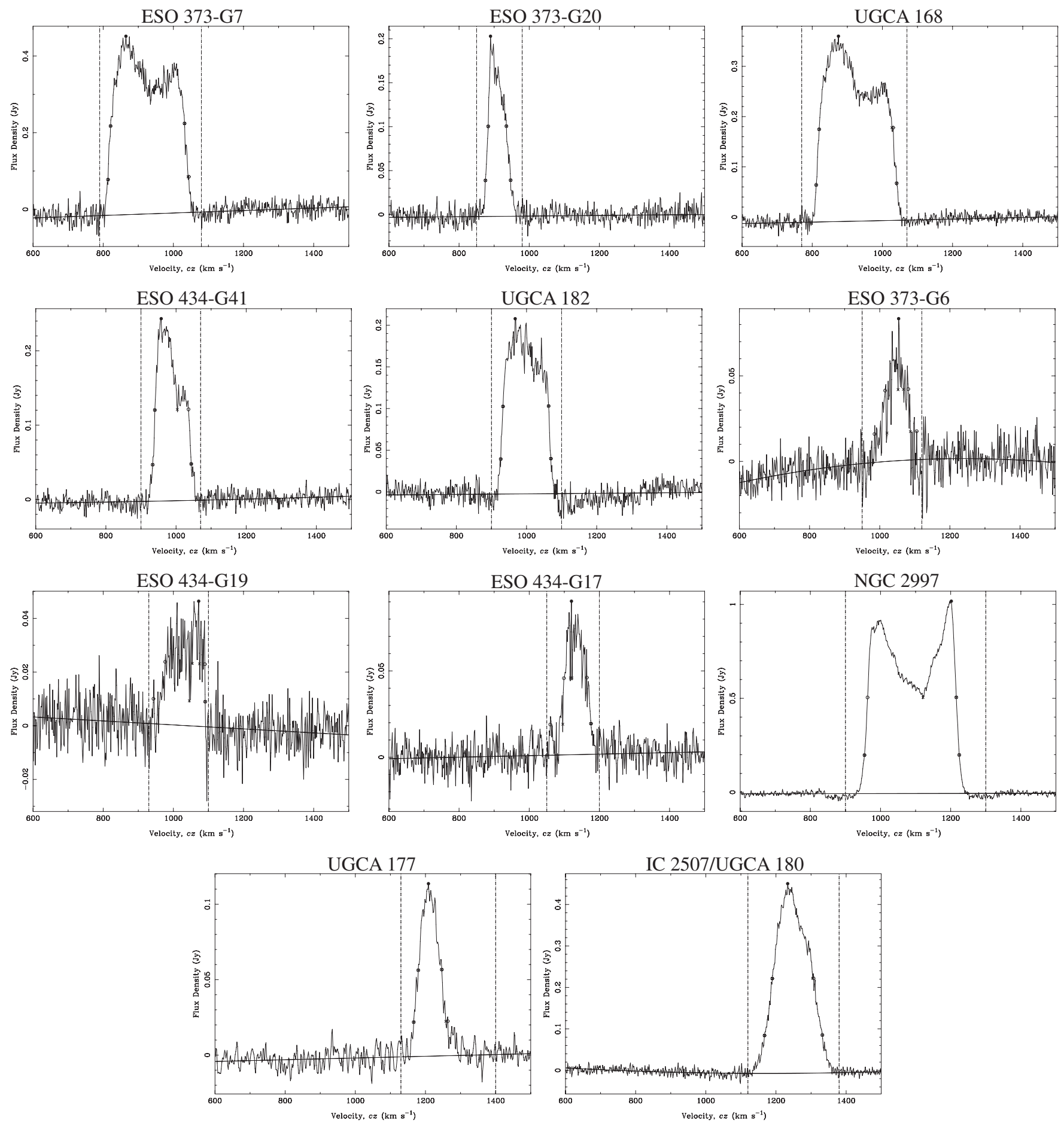

Figure 4. Same as Figure 1, but for LGG 180 group galaxies.

galaxies were all behind the groups and previously detected by HIPASS (Meyer et al. 2004). Of the 105 sources, 15 had previously been observed with the ATCA or Very Large Array (VLA) with similar resolution and equal or better sensitivity than our original observations. Data cubes for IC 1959, ESO 348-G9, IC 5332, ESO 249-G35, ESO 249-G36, IC 2000, NGC 5084, and ESO 576-G40 came from project C 934 courtesy of Emma Ryan-Weber. Data for NGC 2997 comes from the Giant Metreware Radio Telescope (GMRT) and ATCA and were discussed in detail in Hess et al. (2009). The remaining archival data was taken from the ATCA or VLA archives and re-reduced.
The galaxies and projects with archival ATCA data are as follows: ESO 347-G29 (C073), NGC 1433 (C305), NGC 1448 (C295, C419), IC 1986 (C631, C942), UGCA 289 (C1046), NGC 5068 (C892), and ESO 575-G61 (C894). Those with VLA data are DDO 146 (AD474) and UGCA 320 (AC320).

We observed the remaining 90 sources between 2002 October and 2005 March using a compact configuration with baselines shorter than $750 \mathrm{~m}$ yielding beams of $\sim 1^{\prime}-2^{\prime}$, with the exception of galaxies toward the equatorial group LGG 293. For those sources we used the $\mathrm{H} 214 \mathrm{C}$ configuration, which utilizes the north spur of the ATCA and has a maximum baseline of $214 \mathrm{~m}$ 

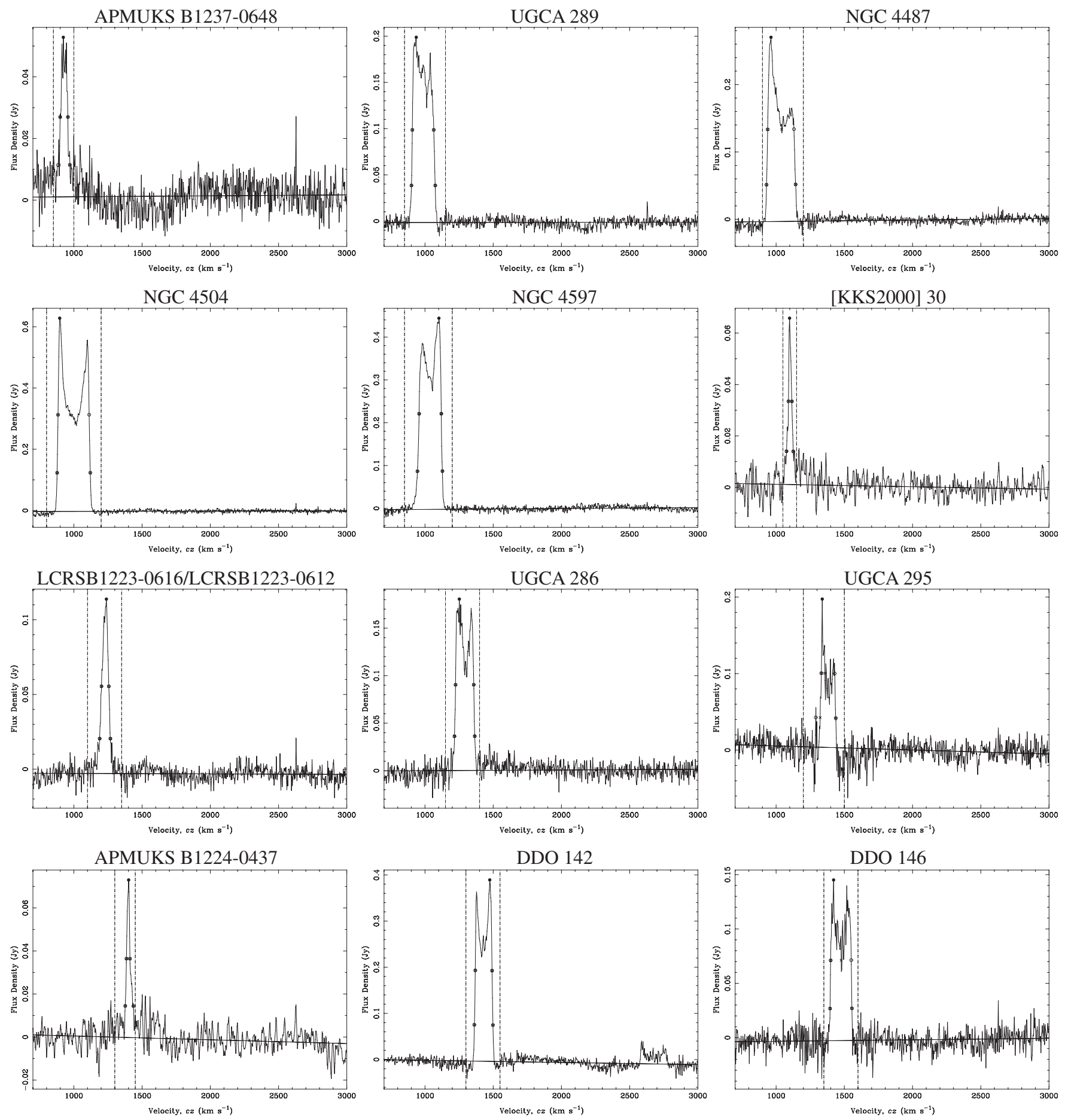

Figure 5. Same as Figure 1, but for LGG 293 group galaxies.

producing a beam of $\sim 2^{\prime}-3^{\prime}$. Sources were observed to at least the same point-source sensitivity as the Parkes observations, $\sim 4$ mJy beam $^{-1}$.

More details on all the observations, data reduction, and source-finding can be found in Paper I.

\section{SURVEY RESULTS}

\subsection{Reliability and Completeness}

Our ATCA observations served two purposes. The primary purpose was to establish the reality of the putative Parkes detections, while the secondary purpose was to identify any
H I-rich galaxies that were confused at the Parkes resolution. Our original and archival interferometer observations confirmed the reality of 106 Parkes detections and revealed an additional four dwarf galaxies behind the target groups that were confused in the original Parkes data. As such our current sample of 110 $\mathrm{H}$ I-rich galaxies is $100 \%$ reliable.

The completeness, which is a necessary measure of a survey if one wishes to construct mass functions, is far more complicated and is discussed in detail in Paper I. Figure 3 of Paper I shows the completeness as a function of the linewidth and integrated signal-to-noise ratio of the source based on the fake sources that were inserted into our Parkes data cubes. In Paper I, we 

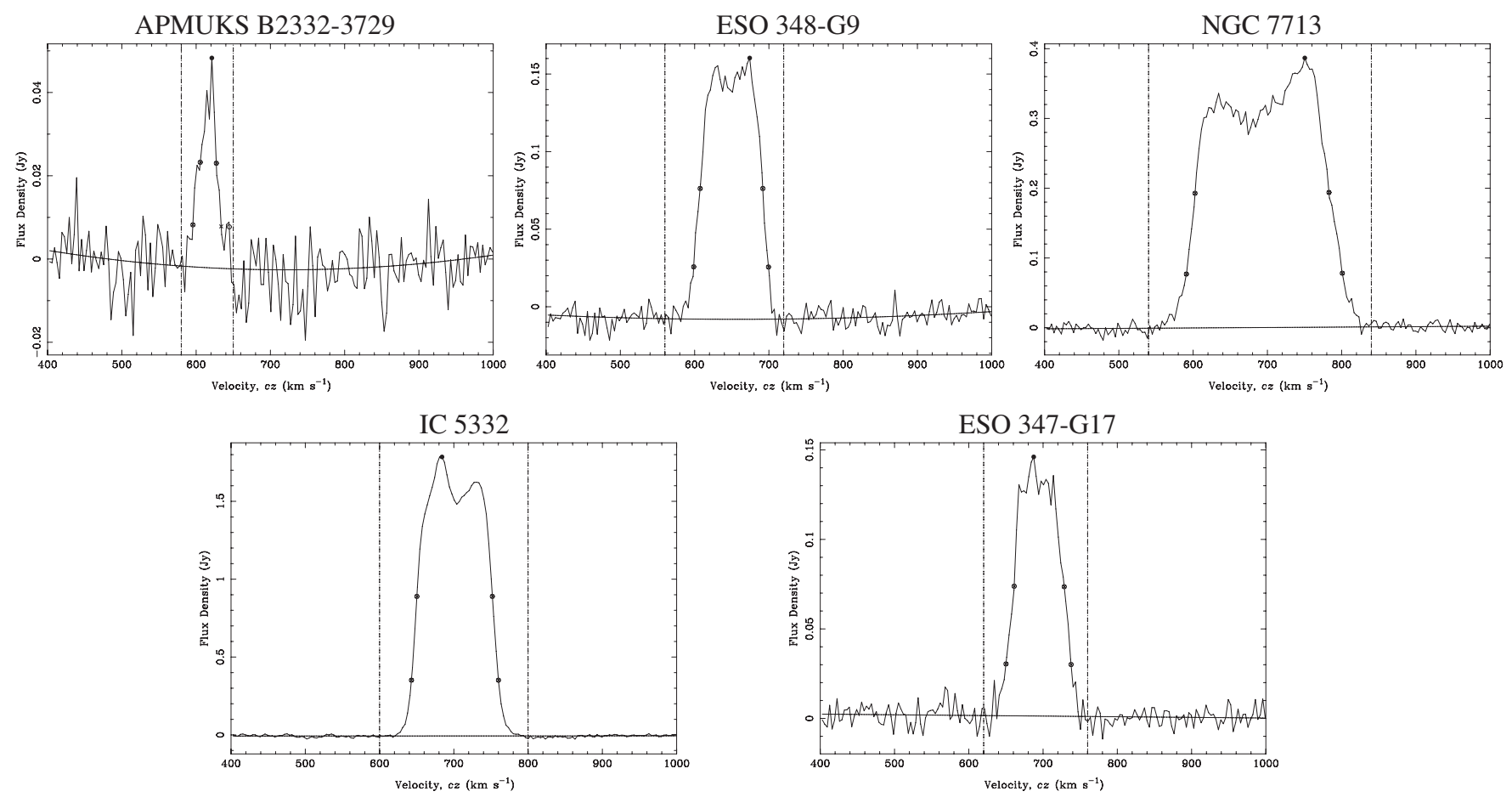

Figure 6. Same as Figure 1, but for LGG 478 group galaxies.
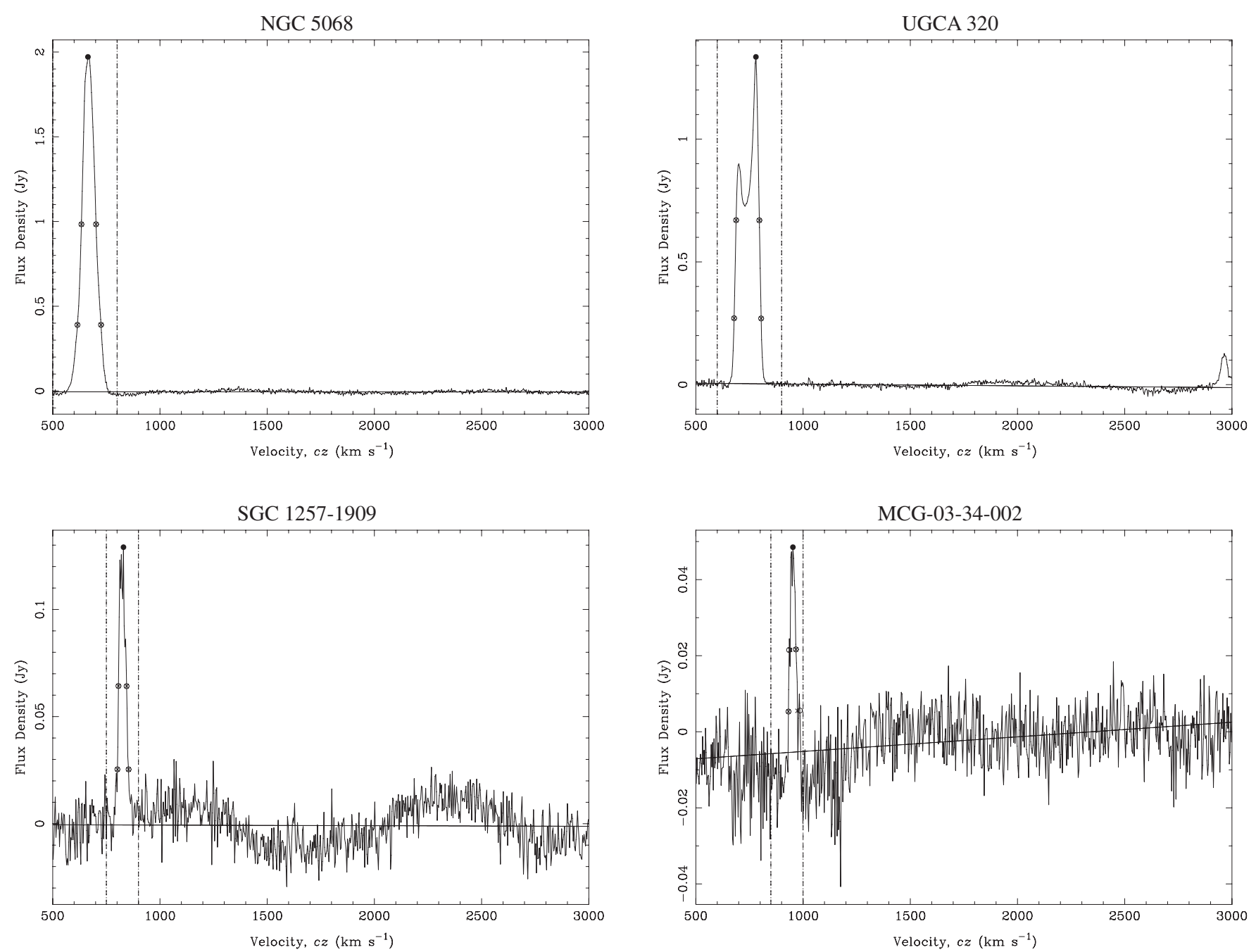

Figure 7. Same as Figure 1, but for HIPASS group galaxies. 

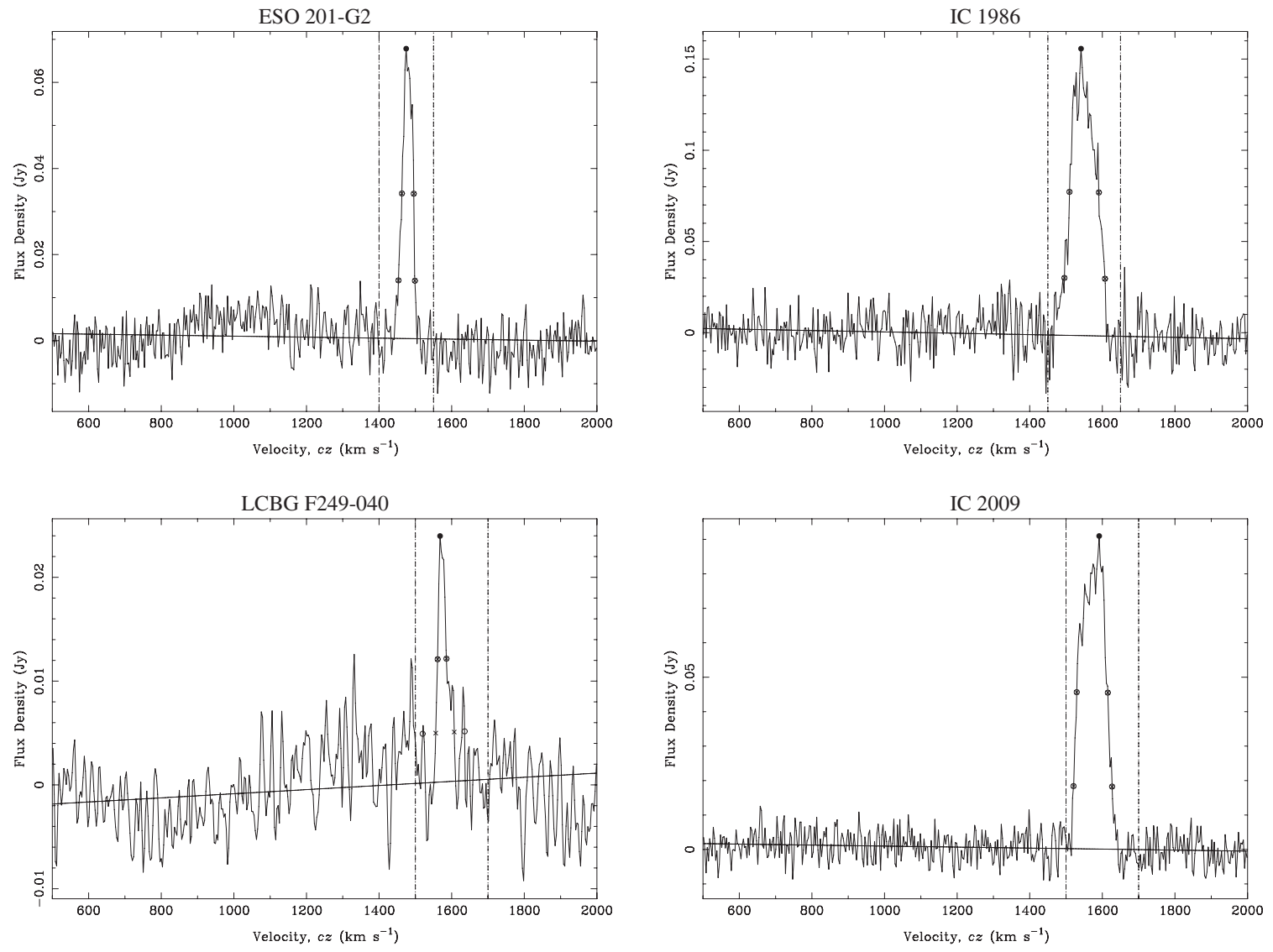

Figure 8. Same as Figure 1, but for galaxies behind LGG 106.
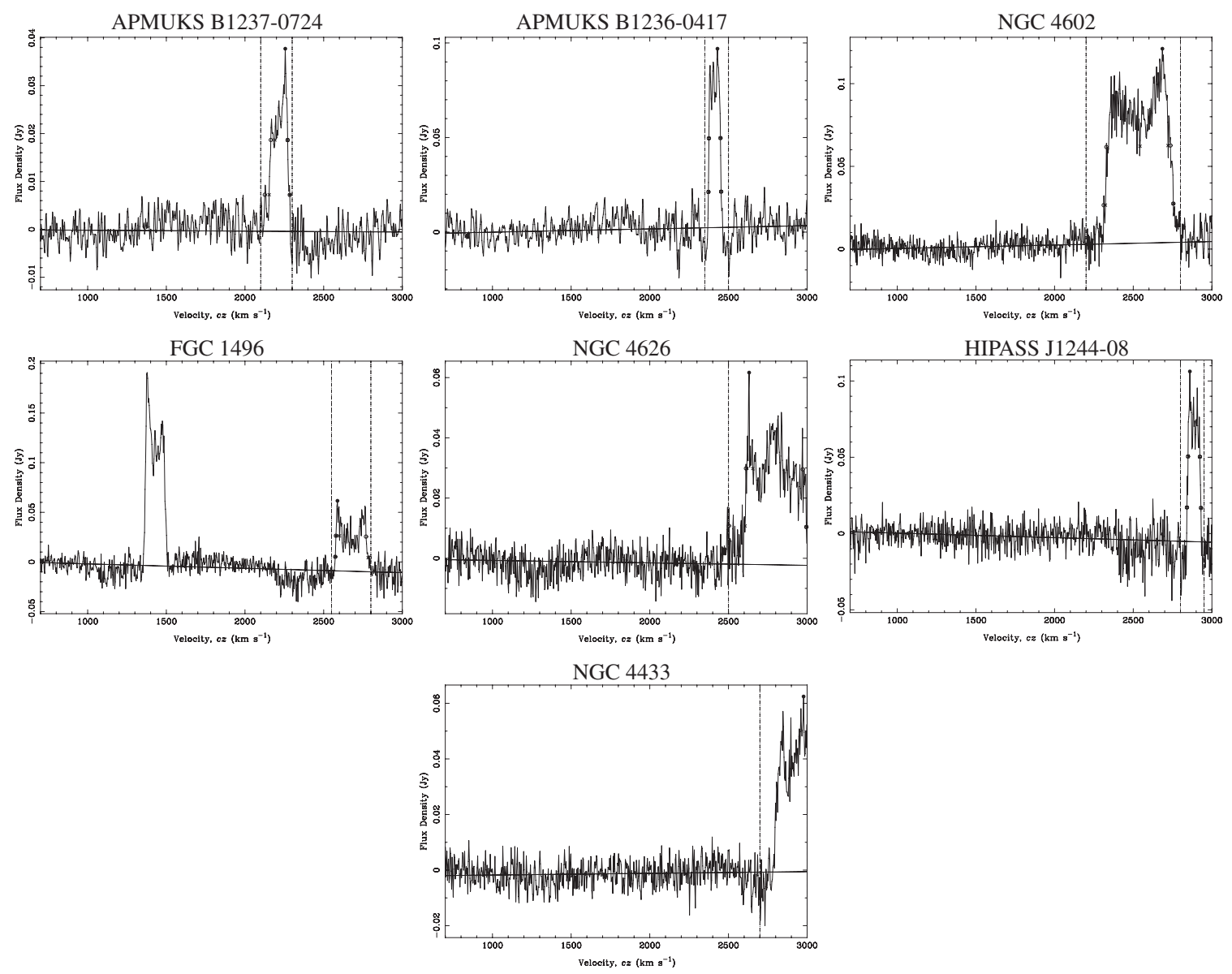

Figure 9. Same as Figure 1, but for galaxies behind LGG 293. 

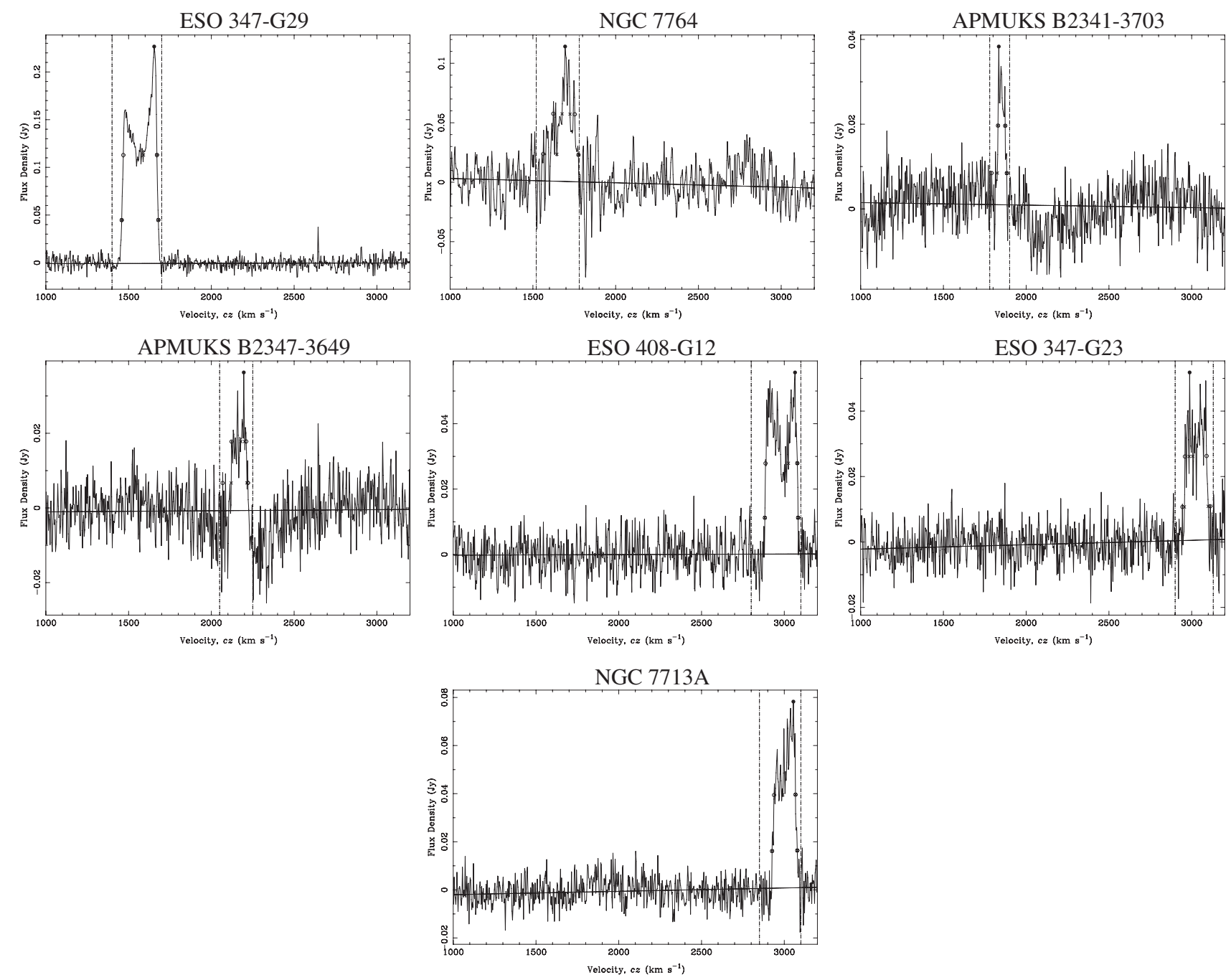

Figure 10. Same as Figure 1, but for galaxies behind LGG 478.

showed that the completeness we inferred was well described by the completeness function for HIPASS from Zwaan et al. (2004) after adjusting it for the different channel widths and noise levels of the two surveys. As in Paper I, we use that scaled completeness function in this paper to derive the HIMF and CVDF.

\subsection{Measuring Galaxy Properties}

Basic source parameters, such as position, recessional velocity, integrated H I flux, and linewidth were measured from the Parkes data using the MBSPECT task in MIRIAD. While searching the cubes, initial positions and velocities were determined for each source. Using MBSPECT, we inspected the cube at these positions to determine a velocity range to fit a firstor a second-order spectral baseline (for any residual shape not removed in the reduction process). MBSPECT takes an input position and velocity and a range of velocities to fit a baseline and measure the $\mathrm{H}$ i profile. It then creates a moment map over the latter range and fits a Gaussian to determine the central position of the source. MBSPECT then forms the spectrum in a $28^{\prime} \times 28^{\prime}$ box centered at this position. For weak sources, the spectra are Hanning smoothed. The resulting spectrum is then robustly integrated and the velocity width at the first and last crossings of $20 \%$ of the peak flux are identified. Typically, we chose the maximum width here, but when this was corrupted by noise spikes, the minimum or an average of the minimum and maximum width was chosen instead. For the two galaxies behind LGG 293 that are at the edge of the observed band, we can only achieve lower limits or highly uncertain estimates of the linewidth and integrated fluxes. The Parkes H I spectra of all detections, including indications of the peak flux as well as the $50 \%$ and $20 \%$ crossings, are shown in Figures 1-12. Where there were multiple galaxies within the Parkes beamwidth, we used the ATCA data to measure the galaxy properties. This was done in the same fashion, except the position was fixed on the known location of the emission and the box in which the line was measured was defined to tightly enclose the visible emission.

We can estimate the errors for our measured parameters by comparing real and measured values for the detected fake sources (see Paper I for details on the fake sources) and by comparing our measured parameters with those in HICAT (Meyer et al. 2004). For the fake sources, our position uncertainty is 2 '. For the remainder of the parameters, after discarding pathological outliers (more than $10 \sigma$, discussed below), $V, W_{20}$, and $S_{\text {int }}$ show a scatter of $2 \mathrm{~km} \mathrm{~s}^{-1}, 4 \mathrm{~km} \mathrm{~s}^{-1}$, and $0.3 \mathrm{Jy} \mathrm{km} \mathrm{s}^{-1}$, respectively. This is much better than the errors for HICAT (Zwaan et al. 2004), even though our fake sources also tend to be fainter, on average, than Zwaan et al.'s. For those sources with large discrepancies, they are mostly faint sources where noise spikes 

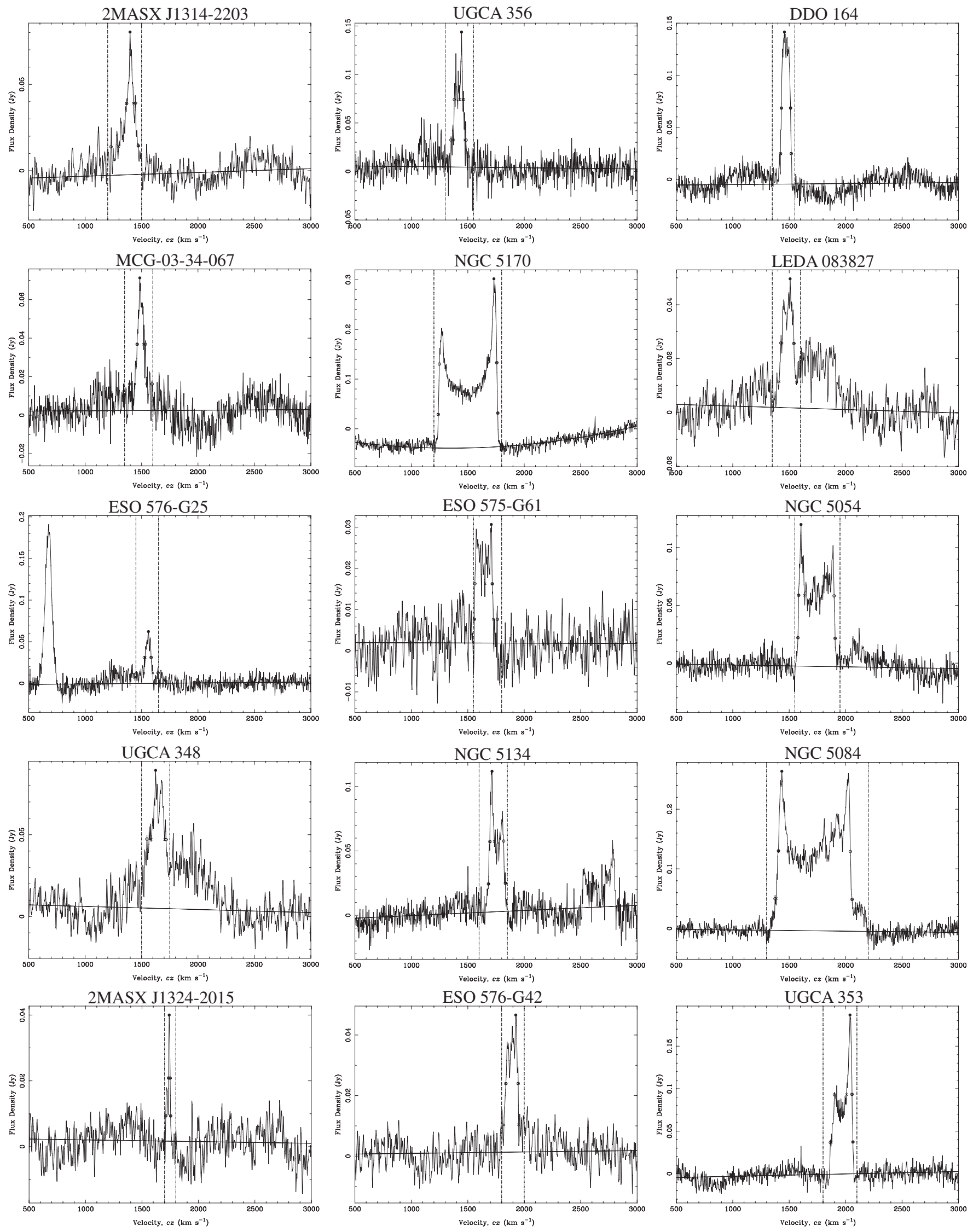

Figure 11. Same as Figure 1, but for galaxies behind the HIPASS group. 

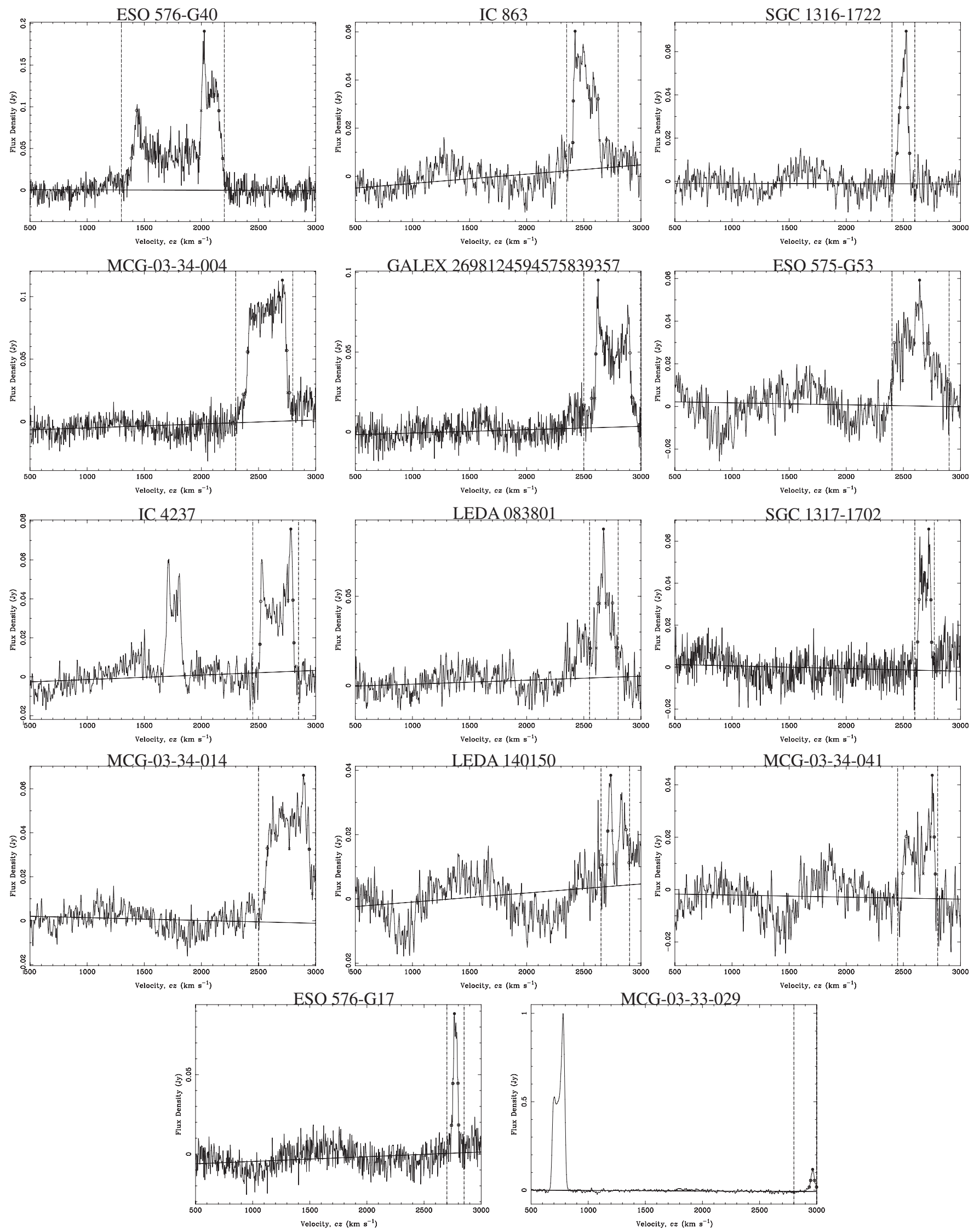

Figure 12. Same as Figure 1, but for the remaining galaxies behind the HIPASS group. 


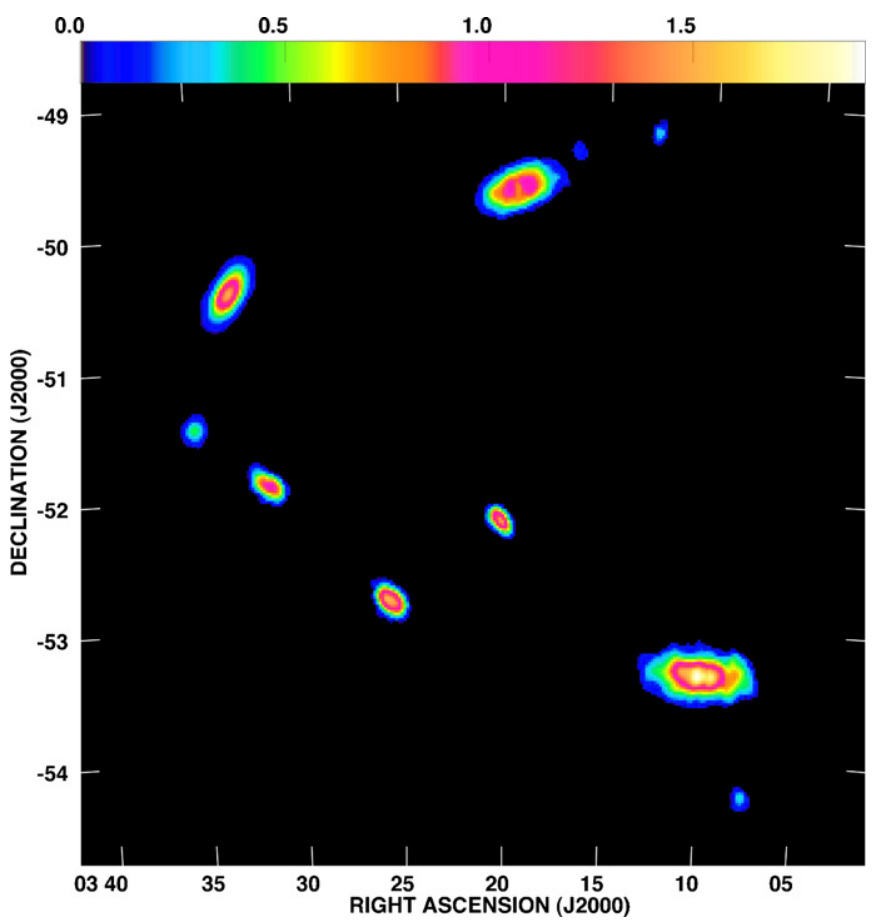

Figure 13. Total $\mathrm{H}_{\mathrm{I}}$ intensity maps of group galaxies in LGG 93 on the same intensity scale (in units of $10^{21} \mathrm{~cm}^{-2}$ ). The galaxies have been placed at their correct locations, but have been scaled up in size by a factor of five.

(A color version of this figure is available in the online journal.)

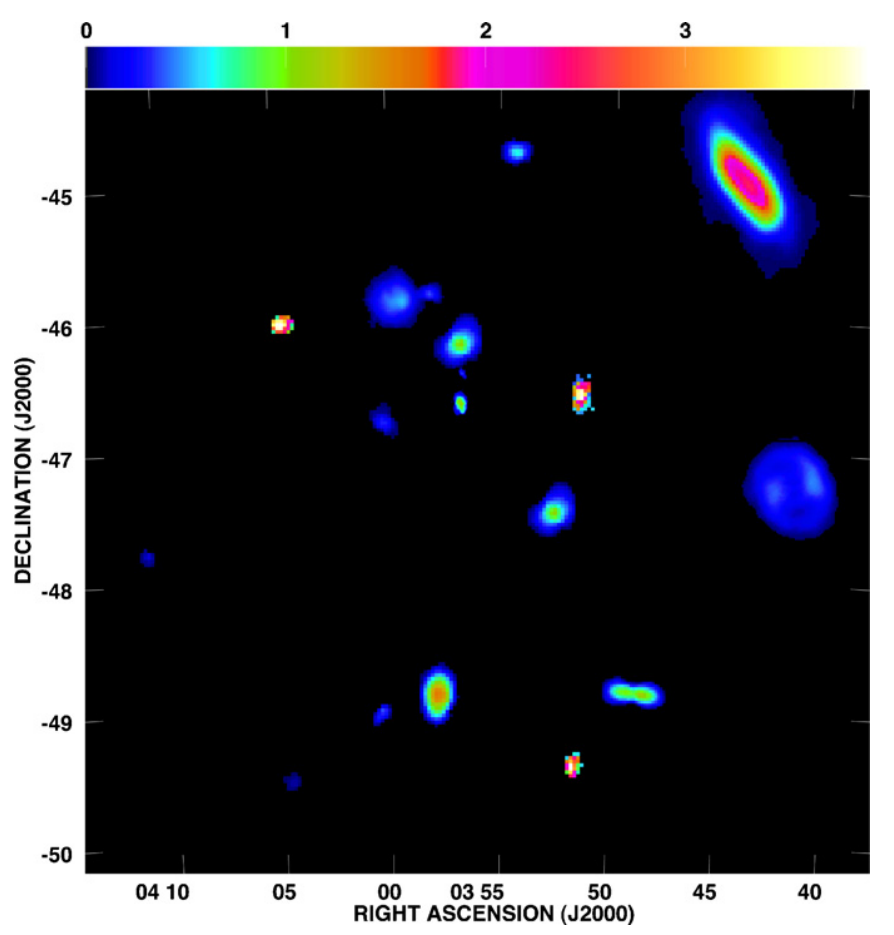

Figure 14. Same as Figure 13, but for LGG 106

(A color version of this figure is available in the online journal.)

are artificially broadening the velocity width measurements or sources with poor baseline fits.

For the brighter galaxies in our sample, we can also compare our measured parameters with those from HICAT (Meyer et al. 2004). There are a total of 65 sources in our survey that are also in HICAT. The positional uncertainty is $2^{\prime}$ and the robust standard deviation, after discarding those sources more than $10 \sigma$

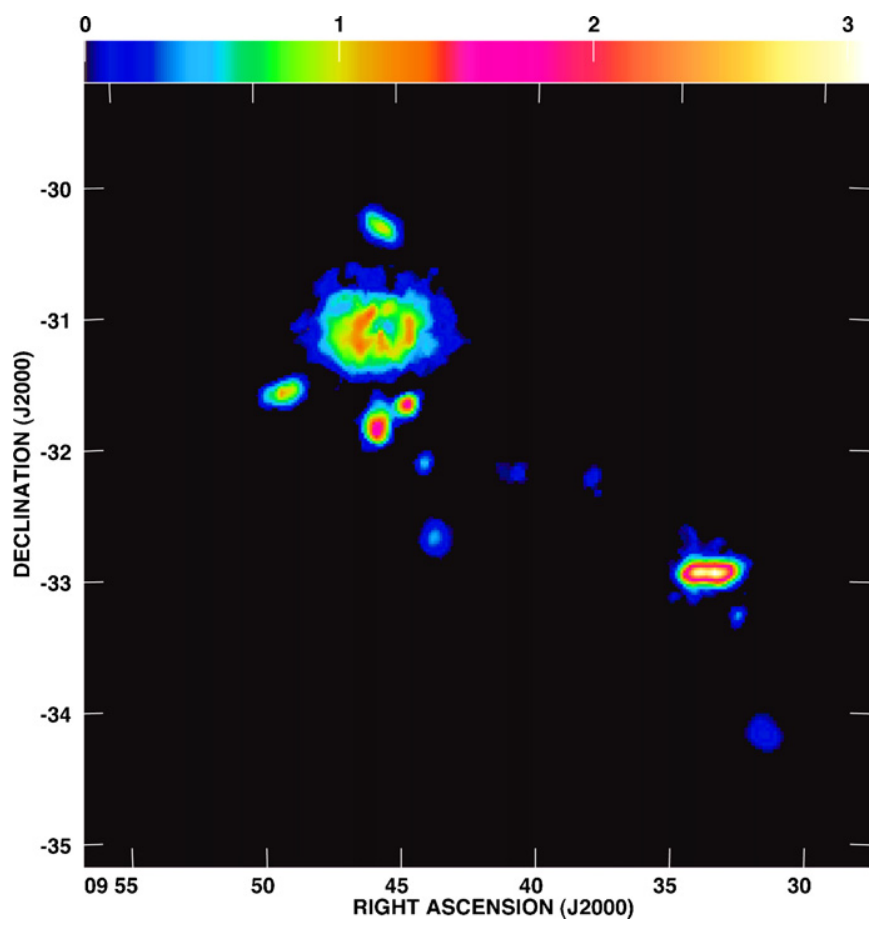

Figure 15. Same as Figure 13, but for LGG 180.

(A color version of this figure is available in the online journal.)

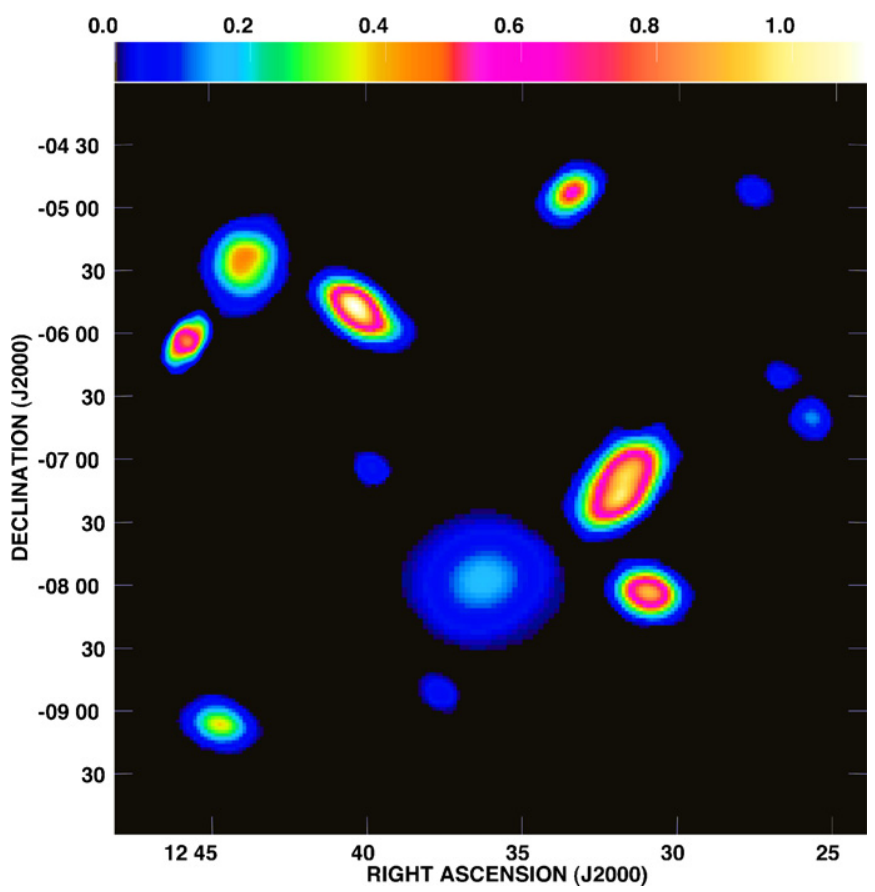

Figure 16. Same as Figure 13, but for LGG 293.

(A color version of this figure is available in the online journal.)

from the mean, of $V, W_{20}$, and $S_{\text {int }}$ are $1 \mathrm{~km} \mathrm{~s}^{-1}, 4 \mathrm{~km} \mathrm{~s}^{-1}$, and $0.9 \mathrm{Jy} \mathrm{km} \mathrm{s}^{-1}$. For $W_{20}$, the HICAT widths are systematically larger by $\sim 15 \mathrm{~km} \mathrm{~s}^{-1}$ due to the coarser velocity resolution of HIPASS. The outliers for the HICAT sources are mostly faint sources or those with bad baselines, as for the fake sources, but there was at least one source that was partly confused with another galaxy. Overall, we feel confident that we can accurately measure the properties of the galaxies from their H i spectra. 


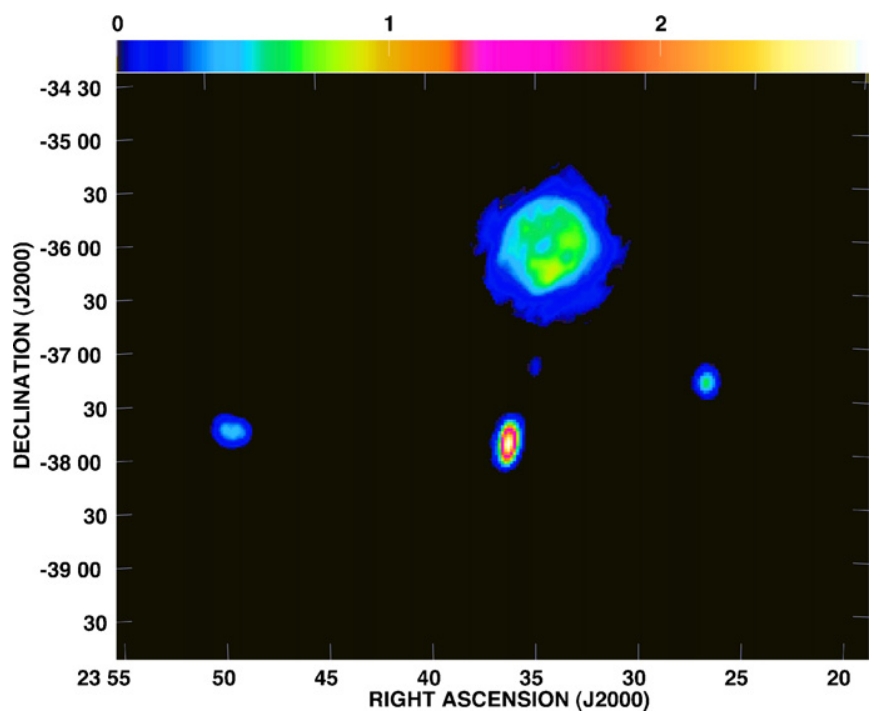

Figure 17. Same as Figure 13, but for LGG 478.

(A color version of this figure is available in the online journal.)

Tables 1 and 2 list the measured $\mathrm{HI}$ properties of the confirmed group and background galaxies.

\subsection{Group Properties}

A total of 31 galaxies detected in our Hi survey were previously identified by Garcia (1993) to be associated with the six groups we observed, but how many of the $110 \mathrm{H} \mathrm{I-rich}$ galaxies we detected are also associated with the groups? To answer this question, we used an iterative process. Starting with the optical group velocity and velocity dispersion, we identified those $\mathrm{HI}$-detected galaxies within three times the velocity dispersion. The mean recession velocity and velocity dispersion is then recalculated and new group members are identified within $3 \sigma$ of the central velocity. The process is repeated until both values have changed by less than $1 \%$. The derived values are listed in Table 3 . We characterize the radial extent of each group in a few different ways. The diameter of each group is taken to be twice the projected separation of the most distant galaxy from the group center. As we assumed that the groups did not extend beyond our survey area, the diameters tend to be comparable to the diagonal across the survey area. We also calculated the mean projected separation between group galaxies, and the projected radial dispersion of the group galaxies from the optically defined group center. Using this approach, we identified a total of 61 group galaxies in the six groups. Overall, our survey roughly doubled the number of group members found by Garcia (1993). To illustrate the relative locations of the group galaxies, Figures 13-18 show the H I total intensity (moment 0) maps of each group galaxy in its proper location, but scaled up in size by a factor of five.

For each group, we calculated a mass, first assuming that they were virialized:

$$
M_{\mathrm{vir}}=\frac{3 \pi(N-1)}{2 G} \frac{\Sigma v_{i}^{2}}{\Sigma 1 / R_{i j}}
$$

from Heisler et al. (1985), where $R_{i j}$ is the projected separation between a pair of galaxies, $v_{i}$ is the velocity difference between the galaxy and the group velocity, and $N$ is the number of group members. If the groups are not virialized, then we can use the

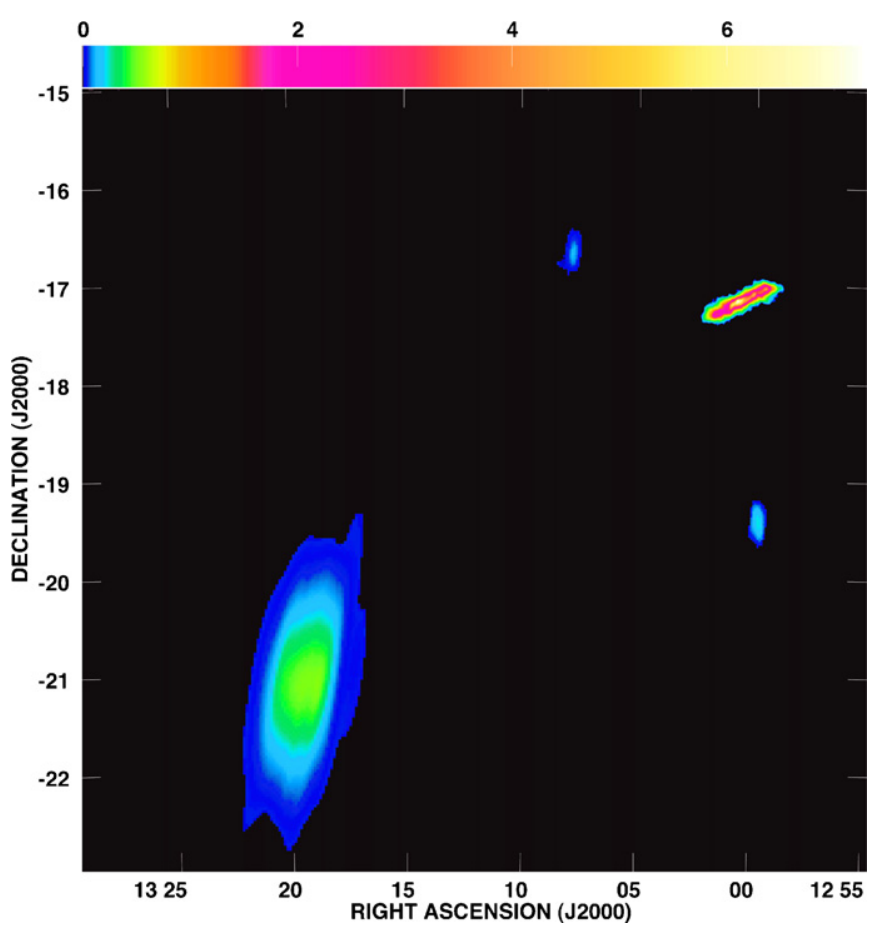

Figure 18. Same as Figure 13, but for the HIPASS group. (A color version of this figure is available in the online journal.)

projected-mass estimator to infer their masses:

$$
M_{\mathrm{pm}}=\frac{32 \Sigma v_{i}^{2} R_{i}}{\pi G(N-1)},
$$

where isotropic orbits are assumed and $R_{i}$ is the separation between the galaxy and the group center (Heisler et al. 1985). For either mass, we are able to calculate the zero-velocity surface for the bound group:

$$
R_{0}=\left(\frac{8 G T^{2} M}{\pi^{2}}\right)^{1 / 3},
$$

where $M$ is the mass and $T$ is the age of the group (Sandage 1986), taken to be $13.7 \mathrm{Gyr}$, the age of the universe (Spergel et al. 2003).

In Table 3, we list the derived group properties for each of the six groups. Our selection of the groups as analogs to the Local Group was based purely on the morphology of the galaxies-group members are only spirals and irregulars, the group members being widely spaced, and the absence of detectable intra-group X-ray emission. From Table 3, we see that all of the groups are very similar to each other in terms of extent, morphology, and derived masses. Their average velocity dispersion is $133 \pm 59 \mathrm{~km} \mathrm{~s}^{-1}$; their average diameter is $1.1 \pm$ $0.2 \mathrm{Mpc}$; and the average separation of the galaxies in the groups is $525 \pm 85 \mathrm{kpc}$. For comparison, we used the measured distances and positions of Local Group galaxies with $M_{\mathrm{HI}} \geqslant$ $10^{7} M_{\odot}$ to calculate a group diameter of $\sim 3.8 \mathrm{Mpc}$, a mean galaxy separation of $\sim 1.3 \mathrm{Mpc}$, and a radial dispersion of 530 kpc (Mateo 1998; van den Bergh 2000). van den Bergh (2000) report that $\sigma_{v}=61 \pm 8 \mathrm{~km} \mathrm{~s}^{-1}$ for the Local Group. If the groups are all virialized, their median mass is $6 \times 10^{12} M_{\odot}$ with an average zero-velocity surface at $1.5 \pm 0.8 \mathrm{Mpc}$. If the groups are not virialized, then we derive a median mass of $1.3 \times 10^{12} M_{\odot}$ with an average zero-velocity surface of $2.1 \pm$ 
Table 1

Group Galaxy H I Detections

\begin{tabular}{|c|c|c|c|c|c|c|}
\hline$\overline{\text { Group }}$ & $\begin{array}{l}\text { Galaxy }^{\mathrm{a}} \\
\end{array}$ & $\alpha(2000)^{\mathrm{b}}$ & $\delta(2000)^{\mathrm{b}}$ & $\begin{array}{c}V_{\odot}^{\mathrm{c}} \\
\left(\mathrm{km} \mathrm{s}^{-1}\right)\end{array}$ & $\begin{array}{c}W_{20} \mathrm{c}^{-} \\
\left(\mathrm{km} \mathrm{s}^{-1}\right)\end{array}$ & $\begin{array}{c}S_{\text {int }}{ }^{\mathrm{c}} \\
\left(\mathrm{Jy} \mathrm{km} \mathrm{s}^{-1}\right)\end{array}$ \\
\hline \multirow[t]{10}{*}{ LGG 93} & NGC 1311 & 032007.4 & -521117 & $573 \pm 2$ & $104 \pm 4$ & $\overline{14.0 \pm 0.1}$ \\
\hline & IC 1959 & 033311.8 & -502431 & $639 \pm 1$ & $149 \pm 2$ & $28.2 \pm 0.1$ \\
\hline & ESO 200-G45 & 033501.2 & -512709 & $1026 \pm 4$ & $52 \pm 8$ & $3.65 \pm 0.08$ \\
\hline & IC 1914 & 031925.2 & -493611 & $1029 \pm 1$ & $215 \pm 2$ & $44.3 \pm 0.1$ \\
\hline & LSBG F200-023 & 031628.4 & -492402 & $1045 \pm 5$ & $88 \pm 10$ & $2.2 \pm 0.2$ \\
\hline & IC 1954 & 033132.1 & -515417 & $1062 \pm 2$ & $231 \pm 4$ & $20.2 \pm 0.2$ \\
\hline & IC 1896 & 030752.6 & -541301 & $1076 \pm 10$ & $118 \pm 20$ & $2.7 \pm 0.2$ \\
\hline & IC 1933 & 032539.5 & -524704 & $1060 \pm 4$ & $208 \pm 8$ & $24.6 \pm 0.1$ \\
\hline & NGC 1249 & 031004.6 & -532001 & $1073 \pm 1$ & $238 \pm 2$ & $99.1 \pm 0.2$ \\
\hline & AM 0311-492 & 031242.8 & -491058 & $1308 \pm 5$ & $64 \pm 10$ & $1.6 \pm 0.1$ \\
\hline \multirow[t]{17}{*}{ LGG 106} & APMUKS B0403-4939 & 040438.0 & -493052 & $854 \pm 2$ & $43 \pm 4$ & $0.90 \pm 0.07$ \\
\hline & ESO 249-G36 & 035915.6 & -455214 & $898 \pm 1$ & $80 \pm 2$ & $15.41 \pm 0.09$ \\
\hline & IC 2000 & 034908.0 & -485126 & $980 \pm 1$ & $280 \pm 2$ & $31.9 \pm 0.1$ \\
\hline & IC 2004 & 035143.8 & -492512 & $1003 \pm 2$ & $126 \pm 4$ & $1.3 \pm 0.1$ \\
\hline & AM $0358-465$ & 035956.6 & -464658 & $1006 \pm 2$ & $84 \pm 4$ & $3.71 \pm 0.09$ \\
\hline & ESO 249-G35 & 035856.3 & -455135 & $1030 \pm 3$ & $128 \pm 6$ & $5.3 \pm 0.1$ \\
\hline & NGC 1433 & 034200.4 & -471327 & $1076 \pm 1$ & $184 \pm 2$ & $31.1 \pm 0.1$ \\
\hline & 6dF J0351-4635 & 035133.2 & -463549 & $1029 \pm 1$ & $59 \pm 2$ & $0.86 \pm 0.09$ \\
\hline & ESO 201-G14 & 040027.5 & -490139 & $1052 \pm 2$ & $167 \pm 4$ & $8.2 \pm 0.1$ \\
\hline & NGC 1493 & 035727.9 & -461220 & $1053 \pm 1$ & $119 \pm 2$ & $41.2 \pm 0.1$ \\
\hline & NGC 1494 & 035743.7 & -485422 & $1131 \pm 1$ & $183 \pm 2$ & $28.9 \pm 0.1$ \\
\hline & NGC 1483 & 035247.3 & -472838 & $1149 \pm 1$ & $155 \pm 2$ & $19.5 \pm 0.2$ \\
\hline & ESO 201-G23 & 041052.8 & -474710 & $1197 \pm 2$ & $85 \pm 4$ & $2.51 \pm 0.09$ \\
\hline & ESO 249-G32 & 035721.6 & -462205 & $1039 \pm 2^{\mathrm{d}}$ & $72 \pm 7^{\mathrm{d}}$ & $5.3 \pm 0.1^{\mathrm{d}}$ \\
\hline & APMUKS B0355-4643 & 035708.2 & -463500 & $1169 \pm 2^{\mathrm{d}}$ & $91 \pm 13^{\mathrm{d}}$ & $2.6 \pm 0.1^{\mathrm{d}}$ \\
\hline & NGC 1448 & 034431.0 & -443834 & $1162 \pm 4$ & $403 \pm 8$ & $20.6 \pm 0.3$ \\
\hline & ESO 250-G5 & 040436.6 & -460212 & $1217 \pm 2$ & $49 \pm 4$ & $0.25 \pm 0.10$ \\
\hline \multirow[t]{12}{*}{ LGG 180} & ESO 373-G7 & 093245.6 & -331440 & $929 \pm 2$ & $230 \pm 4$ & $79.8 \pm 0.2$ \\
\hline & ESO 373-G20 & 094336.1 & -324435 & $911 \pm 2$ & $72 \pm 4$ & $9.4 \pm 0.1$ \\
\hline & UGCA 168 & 093323.0 & -330203 & $926 \pm 2$ & $229 \pm 4$ & $61.9 \pm 0.1$ \\
\hline & ESO 434-G41 & 094743.5 & -313013 & $988 \pm 2$ & $110 \pm 4$ & $18.4 \pm 0.1$ \\
\hline & UGCA 182 & 094527.9 & -302034 & $998 \pm 1$ & $143 \pm 2$ & $22.7 \pm 0.1$ \\
\hline & ESO 373-G6 & 093151.5 & -340813 & $1048 \pm 8$ & $93 \pm 16$ & $3.7 \pm 0.1$ \\
\hline & ESO 434-G19 & 094044.2 & -321345 & $1033 \pm 10$ & $129 \pm 8$ & $5.1 \pm 0.1$ \\
\hline & ESO 434-G17 & 093757.4 & -321720 & $1132 \pm 4$ & $98 \pm 8$ & $4.88 \pm 0.09$ \\
\hline & NGC 2997 & 094543.8 & -311159 & $1089 \pm 1$ & $270 \pm 2$ & $191.2 \pm 0.2$ \\
\hline & UGCA 177 & 094404.1 & -321007 & $1212 \pm 2$ & $97 \pm 4$ & $7.0 \pm 0.2$ \\
\hline & IC 2507 & 094433.9 & -314719 & $1248 \pm 7^{\mathrm{d}}$ & $153 \pm 26^{\mathrm{d}}$ & $20.8 \pm 0.1^{\mathrm{d}}$ \\
\hline & UGCA 180 & 094446.8 & -314913 & $1250 \pm 3^{\mathrm{d}}$ & $149 \pm 3^{\mathrm{d}}$ & $33.2 \pm 0.1^{\mathrm{d}}$ \\
\hline \multirow[t]{13}{*}{ LGG 293} & APMUKS B1237-0648 & 123944.7 & -070523 & $928 \pm 2$ & $87 \pm 4$ & $2.67 \pm 0.09$ \\
\hline & UGCA 289 & 123537.1 & -075222 & $988 \pm 2$ & $174 \pm 4$ & $26.9 \pm 0.1$ \\
\hline & NGC 4487 & 123105.3 & -080307 & $1036 \pm 2$ & $213 \pm 4$ & $36.6 \pm 0.1$ \\
\hline & NGC 4504 & 123218.9 & -073355 & $999 \pm 2$ & $243 \pm 4$ & $93.9 \pm 0.1$ \\
\hline & NGC 4597 & 124011.7 & -054817 & $1036 \pm 2$ & $183 \pm 4$ & $61.4 \pm 0.1$ \\
\hline & [KKS2000] 30 & 123736.3 & -085201 & $1101 \pm 1$ & $49 \pm 2$ & $1.7 \pm 0.1$ \\
\hline & LCRSB1223-0616 & 122538.7 & -063330 & $1244 \pm 2^{\mathrm{d}}$ & $54 \pm 3^{\mathrm{d}}$ & $3.0 \pm 0.1^{\mathrm{e}}$ \\
\hline & LCRSB1223-0612 & 122550.5 & -062924 & $1211 \pm 2^{\mathrm{d}}$ & $50 \pm 7^{\mathrm{d}}$ & $1.3 \pm 0.1^{\mathrm{e}}$ \\
\hline & UGCA 286 & 123337.7 & -045312 & $1290 \pm 1$ & $148 \pm 2$ & $20.1 \pm 0.2$ \\
\hline & UGCA 295 & 124455.0 & -090727 & $1380 \pm 4$ & $115 \pm 8$ & $8.3 \pm 0.3$ \\
\hline & APMUKS B1224-0437 & 122729.2 & -045345 & $1406 \pm 2$ & $60 \pm 4$ & $2.2 \pm 0.2$ \\
\hline & DDO 142 & 124404.1 & -054049 & $1430 \pm 1$ & $136 \pm 2$ & $38.0 \pm 0.2$ \\
\hline & DDO 146 & 124541.1 & -060426 & $1476 \pm 1$ & $160 \pm 2$ & $16.3 \pm 0.2$ \\
\hline \multirow[t]{5}{*}{ LGG 478} & APMUKS B2332-3729 & 233505.2 & -371319 & $615 \pm 2$ & $49 \pm 4$ & $1.14 \pm 0.07$ \\
\hline & ESO $348-G 9$ & 234924.7 & -374622 & $649 \pm 1$ & $101 \pm 2$ & $13.5 \pm 0.1$ \\
\hline & NGC 7713 & 233614.4 & -375607 & $696 \pm 2$ & $210 \pm 4$ & $62.4 \pm 0.2$ \\
\hline & IC 5332 & 233427.4 & -360623 & $701 \pm 1$ & $117 \pm 2$ & $168.1 \pm 0.2$ \\
\hline & ESO $347-G 17$ & 232656.3 & -372035 & $694 \pm 1$ & $88 \pm 2$ & $9.32 \pm 0.08$ \\
\hline \multirow[t]{4}{*}{ HIPASS Group } & NGC 5068 & 131853.8 & -210241 & $670 \pm 1$ & $110 \pm 2$ & $133.5 \pm 0.2$ \\
\hline & UGCA 320 & 130036.8 & -170906 & $742 \pm 2$ & $126 \pm 4$ & $107.3 \pm 0.3$ \\
\hline & SGC 1257-1909 & 125956.0 & -192429 & $828 \pm 1$ & $52 \pm 2$ & $4.5 \pm 0.2$ \\
\hline & MCG-3-34-2 & 130756.6 & -164120 & $958 \pm 4$ & $53 \pm 8$ & $1.1 \pm 0.1$ \\
\hline
\end{tabular}

Notes.

a These are the names of the optical counterparts to the H I detection based on a search of NED.

$\mathrm{b}$ Positions are from the ATCA data and have uncertainties of about $10^{\prime \prime}$.

${ }^{c}$ Data are from Parkes H I spectra, except where noted otherwise.

${ }^{\mathrm{d}}$ From ATCA spectrum.

${ }^{\mathrm{e}}$ Parkes H I flux scaled by ratio of ATCA fluxes. 
Table 2

Background Galaxy H I Detections

\begin{tabular}{|c|c|c|c|c|c|c|}
\hline Foreground Group & Galaxy $^{\mathrm{a}}$ & $\alpha(2000)^{b}$ & $\delta(2000)^{\mathrm{b}}$ & $V_{\odot}{ }^{\mathrm{c}}$ & $W_{20}{ }^{\mathrm{c}}$ & $S_{\text {int }}{ }^{\mathrm{c}}$ \\
\hline \multirow[t]{4}{*}{ LGG 106} & ESO 201-G2 & 034842.7 & -482508 & $1476 \pm 2$ & $45 \pm 4$ & $1.9 \pm 0.1$ \\
\hline & IC 1986 & 034034.7 & -452119 & $1551 \pm 2$ & $112 \pm 4$ & $11.4 \pm 0.2$ \\
\hline & LSBG F249-040 & 034934.9 & -463415 & $1581 \pm 4$ & $51 \pm 8$ & $0.63 \pm 0.15$ \\
\hline & IC 2009 & 035334.6 & -485931 & $1574 \pm 2$ & $106 \pm 4$ & $6.87 \pm 0.09$ \\
\hline \multirow[t]{7}{*}{ LGG 293} & APMUKS B1237-0724 & 124016.6 & -074105 & $2219 \pm 4$ & $131 \pm 8$ & $2.9 \pm 0.1$ \\
\hline & APMUKS B1236-0417 & 123901.2 & -043335 & $2413 \pm 2$ & $81 \pm 4$ & $5.6 \pm 0.2$ \\
\hline & NGC 4602 & 124038.1 & -050749 & $2539 \pm 4$ & $432 \pm 8$ & $35.1 \pm 0.2$ \\
\hline & FGC 1496 & 124418.9 & -053212 & $2678 \pm 4$ & $209 \pm 8$ & $8.2 \pm 0.2$ \\
\hline & NGC 4626 & 124227.7 & -065810 & $2800 \pm 8^{\mathrm{d}}$ & $389 \pm 16^{\mathrm{d}}$ & $>13.2 \pm 0.2^{\mathrm{d}}$ \\
\hline & HIPASS J1244-08 & 124512.5 & -082109 & $2886 \pm 2$ & $89 \pm 4$ & $6.1 \pm 0.1$ \\
\hline & NGC 4433 & 122738.9 & -081639 & $\sim 2977^{\mathrm{d}}$ & $\gtrsim 200^{\mathrm{d}}$ & $>8.3 \pm 0.1^{\mathrm{d}}$ \\
\hline \multirow[t]{7}{*}{ LGG 478} & ESO 347-G29 & 233628.2 & -384715 & $1569 \pm 1$ & $221 \pm 2$ & $30.8 \pm 0.1$ \\
\hline & NGC 7764 & 235054.0 & -404359 & $1668 \pm 8$ & $213 \pm 16$ & $9.4 \pm 0.7$ \\
\hline & APMUKS B2341-3703 & 234413.6 & -364626 & $1854 \pm 4$ & $57 \pm 8$ & $1.35 \pm 0.09$ \\
\hline & APMUKS B2347-3649 & 235033.7 & -363311 & $2168 \pm 2$ & $95 \pm 4$ & $0.93 \pm 0.12$ \\
\hline & ESO 408-G12 & 233736.4 & -365904 & $2983 \pm 1$ & $201 \pm 2$ & $7.3 \pm 0.1$ \\
\hline & ESO 347-G23 & 233431.2 & -393158 & $3028 \pm 4$ & $144 \pm 8$ & $4.5 \pm 0.1$ \\
\hline & NGC 7713A & 233709.8 & -374256 & $3002 \pm 2$ & $152 \pm 4$ & $7.2 \pm 0.1$ \\
\hline \multirow[t]{30}{*}{ HIPASS Group } & 2MASX J1314-2203 & 131451.7 & -220430 & $1384 \pm 4$ & $173 \pm 8$ & $6.5 \pm 0.3$ \\
\hline & UGCA 356 & 132636.0 & -221404 & $1418 \pm 4$ & $133 \pm 8$ & $8.7 \pm 0.2$ \\
\hline & DDO 164 & 130617.9 & -173048 & $1470 \pm 1$ & $95 \pm 2$ & $11.0 \pm 0.2$ \\
\hline & MCG-3-34-67 & 132415.4 & -164216 & $1494 \pm 3$ & $101 \pm 6$ & $4.3 \pm 0.2$ \\
\hline & NGC 5170 & 132948.2 & -175740 & $1502 \pm 1$ & $527 \pm 2$ & $79.3 \pm 0.6$ \\
\hline & LEDA 083827 & 131430.6 & -162230 & $1487 \pm 2$ & $153 \pm 4$ & $4.9 \pm 0.2$ \\
\hline & ESO 576-G25 & 131829.8 & -204108 & $1560 \pm 2$ & $79 \pm 4$ & $3.2 \pm 0.2$ \\
\hline & ESO 575-G61 & 130814.9 & -205958 & $1642 \pm 3$ & $168 \pm 6$ & $3.3 \pm 0.2$ \\
\hline & NGC 5054 & 131658.7 & -163836 & $1742 \pm 2$ & $330 \pm 4$ & $23.5 \pm 0.2$ \\
\hline & UGCA 348 & 131951.1 & -221638 & $1617 \pm 8^{\mathrm{e}}$ & $177 \pm 16^{\mathrm{e}}$ & $9.2 \pm 0.5^{\mathrm{e}}$ \\
\hline & NGC 5134 & 132518.6 & -210809 & $1758 \pm 2$ & $150 \pm 4$ & $9.4 \pm 0.2$ \\
\hline & NGC 5084 & 132015.6 & -214953 & $1715 \pm 4$ & $683 \pm 8$ & $106.0 \pm 0.4$ \\
\hline & 2MASX J1324-2015 & 132454.4 & -201745 & $1732 \pm 2$ & $43 \pm 4$ & $0.53 \pm 0.15$ \\
\hline & ESO 576-G42 & 132201.9 & -201319 & $1885 \pm 4$ & $130 \pm 8$ & $4.2 \pm 0.2$ \\
\hline & UGCA 353 & 132441.8 & -194216 & $1964 \pm 4$ & $200 \pm 8$ & $17.7 \pm 0.2$ \\
\hline & ESO 576-G40 & 132043.6 & -220308 & $1787 \pm 4$ & $799 \pm 8$ & $50.8 \pm 0.4$ \\
\hline & IC 863 & 131713.2 & -171507 & $2514 \pm 3^{\mathrm{e}}$ & $244 \pm 6^{f}$ & $5.9 \pm 0.2^{\mathrm{f}}$ \\
\hline & GALEX 2698124594575839357 & 131737.1 & -172141 & $2477 \pm 7^{\mathrm{e}}$ & $112 \pm 13^{\mathrm{e}}$ & $3.2 \pm 0.2^{\mathrm{f}}$ \\
\hline & SGC 1316-1722 & 131855.5 & -173808 & $2499 \pm 1$ & $109 \pm 2$ & $4.7 \pm 0.2$ \\
\hline & MCG-3-34-4 & 130943.3 & -163614 & $2569 \pm 4$ & $405 \pm 8$ & $32.6 \pm 0.3$ \\
\hline & ESO 576-G11 & $131254.7^{\mathrm{g}}$ & $-200129^{\mathrm{g}}$ & $2757 \pm 4$ & $318 \pm 8$ & $17.8 \pm 0.2$ \\
\hline & ESO 575-G53 & 130505.7 & -222249 & $2644 \pm 8$ & $487 \pm 16$ & $12.5 \pm 0.5$ \\
\hline & IC 4237 & $132440.1^{\mathrm{g}}$ & $-211039^{g}$ & $2661 \pm 1$ & $298 \pm 2$ & $11.1 \pm 0.3$ \\
\hline & LEDA 083801 & $131326.3^{\mathrm{g}}$ & $-160330^{\mathrm{g}}$ & $2693 \pm 4$ & $166 \pm 8$ & $7.9 \pm 0.3$ \\
\hline & SGC 1317-1702 & 131955.0 & -171850 & $2686 \pm 2$ & $123 \pm 4$ & $5.2 \pm 0.1$ \\
\hline & MCG-3-34-14 & $131245.1^{\mathrm{g}}$ & $-173221^{\mathrm{g}}$ & $2763 \pm 4$ & $413 \pm 8$ & $19.1 \pm 0.3$ \\
\hline & LEDA 140150 & $131326.3^{\mathrm{g}}$ & $-192421^{\mathrm{g}}$ & $2780 \pm 8$ & $232 \pm 16$ & $3.3 \pm 0.3$ \\
\hline & MCG-3-34-41 & 131706.2 & -161511 & $2636 \pm 2$ & $286 \pm 4$ & $5.6 \pm 0.3$ \\
\hline & ESO 576-G17 & $131502.3^{\mathrm{g}}$ & $-175725^{\mathrm{g}}$ & $2771 \pm 1$ & $62 \pm 2$ & $3.5 \pm 0.1$ \\
\hline & MCG-3-34-29 & $130311.1^{\mathrm{g}}$ & $-171755^{\mathrm{g}}$ & $2966 \pm 1^{\mathrm{d}}$ & $63 \pm 2^{\mathrm{d}}$ & $>4.4 \pm 0.2^{\mathrm{d}}$ \\
\hline
\end{tabular}

Notes.

${ }^{a}$ These are the names of the optical counterparts to the H I detection based on a search of NED.

${ }^{\mathrm{b}}$ Positions are from the ATCA data and have uncertainties of about $10^{\prime \prime}$.

${ }^{c}$ Data are from Parkes H i spectra, except where noted otherwise.

${ }^{\mathrm{d}} \mathrm{H}$ I profile at the edge of bandpass, so values are highly uncertain or only lower limits.

${ }^{\mathrm{e}}$ From ATCA spectrum.

${ }^{\mathrm{f}}$ Parkes Hi flux scaled by ratio of ATCA fluxes.

${ }^{g}$ From Parkes H I data for those galaxies in HICAT and not confirmed by ATCA observations.

$0.8 \mathrm{Mpc}$. The derived sizes and masses of these loose groups, whether these systems are virialized or not, are all similar to the Local Group with $M_{\text {vir }}=(2.3 \pm 0.6) \times 10^{12} M_{\odot}$ and $R_{0}=1.15 \pm 0.15 \mathrm{Mpc}$ (van den Bergh 2000). As such, we remain confident that these groups are good analogs for the Local Group.

\subsection{Galaxy Properties}

Interferometer $\mathrm{H}$ I total intensity (moment 0 ) maps overlaid on optical images for all group galaxies are shown in Figures 19-25. $\mathrm{HI}$ total intensity contours on optical maps for the background galaxies are shown in Figures 26-30. The properties of the 

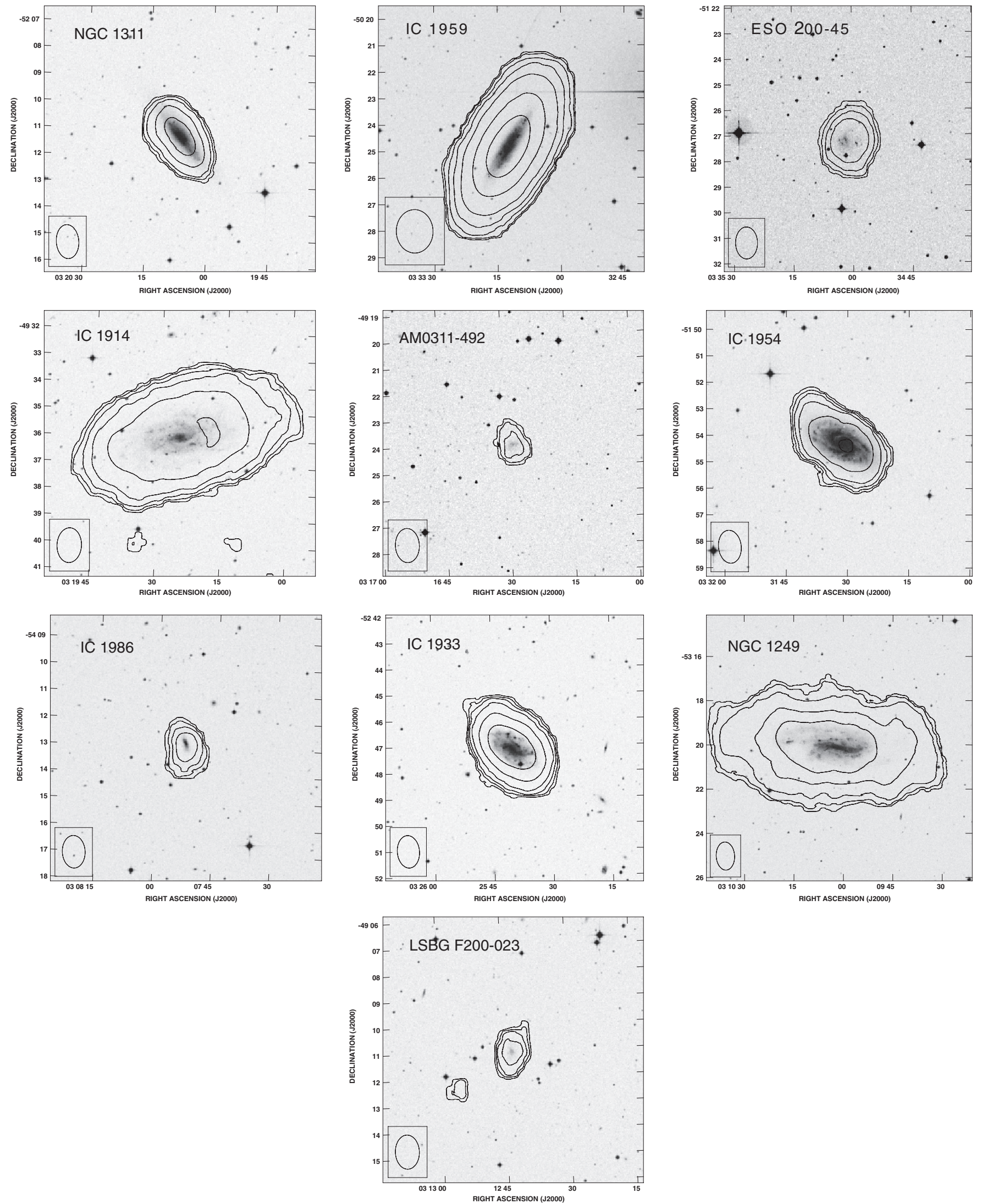

Figure 19. ATCA total H I intensity (moment 0) contours overlaid on second-generation blue Digital Sky Survey gray-scale images for LGG 93 group galaxies. Contour levels are given in Table 4. The beam is shown as the boxed ellipse at the bottom of each image. 

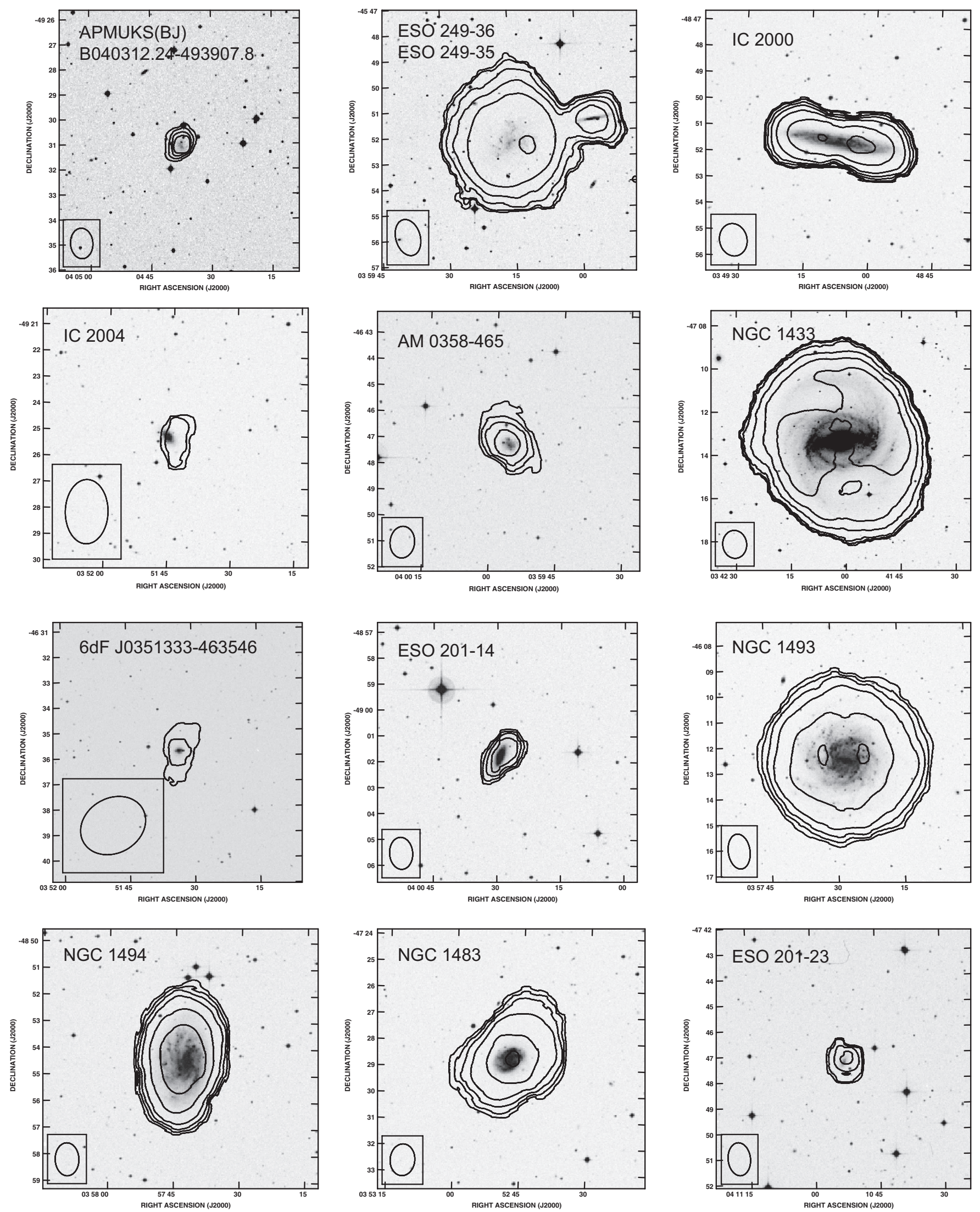

Figure 20. Same as Figure 19, but for LGG 106 group galaxies. 

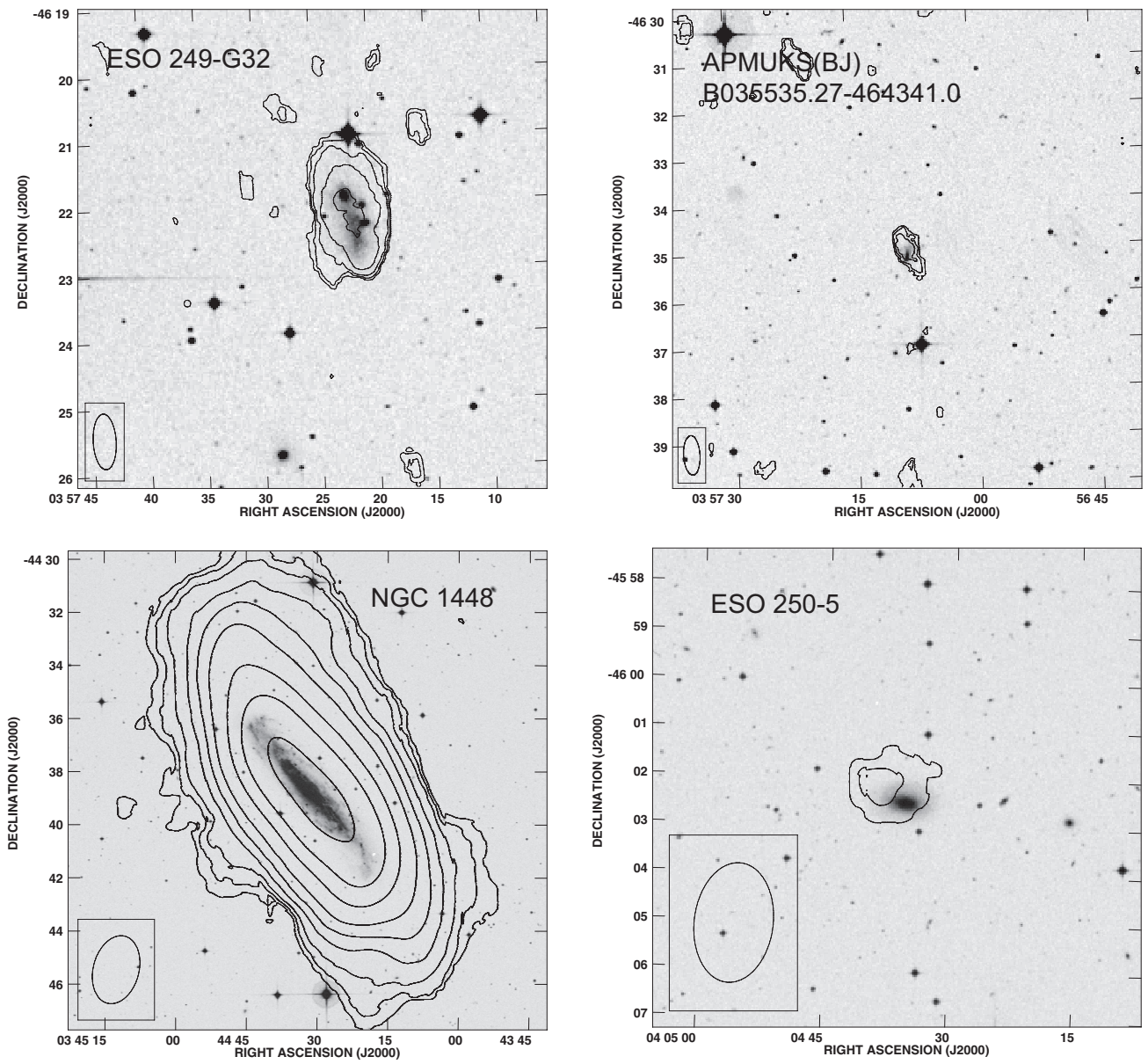

Figure 21. Same as Figure 19, but for the remaining LGG 106 group galaxies.

Table 3

Group Properties

\begin{tabular}{|c|c|c|c|c|c|c|c|c|}
\hline Property & Units & LGG 93 & LGG 106 & LGG 180 & LGG 293 & LGG 478 & HIPASS Group & Local Group ${ }^{\mathrm{a}}$ \\
\hline Distance & $\mathrm{Mpc}$ & 10.9 & 13.8 & 14.8 & 11.1 & 8.6 & 9.1 & $\ldots$ \\
\hline Number of members & & 10 & 17 & 12 & 13 & 5 & 4 & 21 \\
\hline$V_{\odot}^{\mathrm{b}}$ & $\mathrm{km} \mathrm{s}^{-1}$ & 989 & 1061 & 1064 & 1194 & 671 & 800 & $\ldots$ \\
\hline$\sigma_{v}{ }^{\mathrm{c}}$ & $\mathrm{km} \mathrm{s}^{-1}$ & 218 & 101 & 123 & 191 & 38 & 124 & $61 \pm 8^{d}$ \\
\hline Diameter $^{\mathrm{e}}$ & $\mathrm{Mpc}$ & 1.0 & 1.4 & 1.2 & 1.2 & 0.68 & 0.92 & $3.8^{\mathrm{f}}$ \\
\hline Mean galaxy-galaxy separation & $\mathrm{Mpc}$ & 0.57 & 0.58 & 0.49 & 0.56 & 0.35 & 0.60 & 1.3 \\
\hline$\sigma_{r} \mathrm{~g}$ & $\mathrm{Mpc}$ & 0.48 & 0.48 & 0.46 & 0.45 & 0.27 & 0.46 & 0.53 \\
\hline$M_{\mathrm{vir}}^{\mathrm{h}}$ & $10^{12} M_{\odot}$ & $36 \pm 11$ & $4.6 \pm 1.1$ & $7.0 \pm 2.0$ & $11 \pm 3$ & $0.13 \pm 0.06$ & $0.51 \pm 0.25$ & $2.3 \pm 0.6^{\mathrm{d}}$ \\
\hline$R_{0, \mathrm{vir}}{ }^{\mathrm{i}}$ & $\mathrm{Mpc}$ & 2.9 & 1.5 & 1.7 & 2.0 & 0.45 & 0.70 & $1.15 \pm 0.15^{\mathrm{d}}$ \\
\hline$M_{\mathrm{pm}} \mathrm{j}$ & $10^{12} M_{\odot}$ & $38 \pm 12$ & $12 \pm 3$ & $10 \pm 3$ & $34 \pm 9$ & $0.41 \pm 0.18$ & $14 \pm 7$ & $\ldots$ \\
\hline$R_{0, \mathrm{pm}} \mathrm{k}$ & $\mathrm{Mpc}$ & 3.0 & 2.0 & 1.9 & 2.8 & 0.65 & 2.1 & $\cdots$ \\
\hline
\end{tabular}

Notes.

${ }^{\text {a }}$ Only those group members with $M_{\mathrm{H}_{\mathrm{I}}} \geqslant 10^{7} M_{\odot}$ are used to calculate number of members and radii of the Local Group.

${ }^{b}$ The mean recession velocity of the group members.

${ }^{c}$ The rms velocity dispersion of the group members.

${ }^{\mathrm{d}}$ From van den Bergh (2000).

e Twice the projected separation of the most distant group member from the group center.

$\mathrm{f}$ The radial separation of GR8 from the Local Group barycenter.

$\mathrm{g}$ The rms dispersion of the projected radial separations of group galaxies.

${ }^{\mathrm{h}}$ Calculated using Equation (1) from Heisler et al. (1985).

${ }^{\mathrm{i}}$ Calculated using Equation (3) from Sandage (1986) and $M_{\text {vir }}$.

j Calculated using Equation (2) from Heisler et al. (1985).

${ }^{\mathrm{k}}$ Calculated using Equation (3) from Sandage (1986) and $M_{\mathrm{pm}}$. 
Table 4

Interferometer Data for Group Galaxies

\begin{tabular}{|c|c|c|c|c|c|}
\hline \multirow[t]{2}{*}{ Galaxy } & \multirow{2}{*}{$\begin{array}{c}\text { Beam Size } \\
(\operatorname{arcsec})\end{array}$} & \multirow{2}{*}{$\begin{array}{c}\text { Channel Width } \\
\left(\mathrm{km} \mathrm{s}^{-1}\right)\end{array}$} & \multicolumn{2}{|c|}{ Noise } & \multirow{2}{*}{$\begin{array}{l}\text { Contour Levels }{ }^{\mathrm{a}} \\
\left(10^{19} \mathrm{~cm}^{-2}\right)\end{array}$} \\
\hline & & & $\left(\mathrm{mJy}\right.$ beam $\left.^{-1}\right)$ & $\left(10^{19} \mathrm{~cm}^{-2}\right)$ & \\
\hline NGC 1311 & $76 \times 50$ & 6.6 & 5.9 & 1.1 & $5,10,20,50,100$ \\
\hline IC 1959 & $98 \times 83$ & 6.6 & 3.4 & 0.3 & $1,2,5,10,20,50,100$ \\
\hline ESO 200-G45 & $75 \times 51$ & 6.6 & 3.5 & 0.7 & $2,5,10,20,50$ \\
\hline IC 1914 & $78 \times 54$ & 6.6 & 4.0 & 0.7 & $2,5,10,20,50,100$ \\
\hline LSBG F200-023 & $78 \times 54$ & 6.6 & 4.0 & 0.7 & $2,5,10,20,50,100$ \\
\hline IC 1954 & $75 \times 51$ & 6.6 & 3.7 & 0.7 & $2,5,10,20,50,100$ \\
\hline IC 1896 & $73 \times 51$ & 6.6 & 3.7 & 0.7 & $2,5,10,20$ \\
\hline IC 1933 & $75 \times 51$ & 6.6 & 3.8 & 0.7 & $2,5,10,20,50,100$ \\
\hline NGC 1249 & $76 \times 50$ & 6.6 & 5.4 & 1.0 & $5,10,20,50,100$ \\
\hline AM 0311-492 & $78 \times 54$ & 6.6 & 4.0 & 0.7 & $2,5,10,20,50,100$ \\
\hline APMUKS B0403-4939 & $73 \times 54$ & 3.3 & 4.9 & 0.5 & $2,3,4,5$ \\
\hline ESO 249-G36 & $86 \times 57$ & 3.3 & 3.7 & 0.3 & $1,2,5,10,20,50,100$ \\
\hline ESO 249-G35 & $86 \times 57$ & 3.3 & 3.7 & 0.3 & $1,2,5,10,20,50,100$ \\
\hline IC 2000 & $75 \times 63$ & 3.3 & 4.0 & 0.3 & $1,2,5,10,20,50,100$ \\
\hline IC 2004 & $147 \times 98$ & 3.3 & 5.1 & 0.1 & $0.5,1$ \\
\hline AM 0358-465 & $73 \times 57$ & 3.3 & 6.0 & 0.5 & $2,6,10,20$ \\
\hline NGC 1433 & $78 \times 66$ & 6.6 & 1.2 & 0.2 & $0.5,1,2,5,10,20$ \\
\hline 6dF J0351-4635 & $161 \times 130$ & 3.3 & 5.3 & 0.1 & $0.5,1$ \\
\hline ESO 201-G14 & $74 \times 54$ & 3.3 & 7.1 & 0.6 & $2,5,10,20$ \\
\hline NGC 1493 & $82 \times 51$ & 3.3 & 5.8 & 0.5 & $2,5,10,20,50,100$ \\
\hline NGC 1494 & $74 \times 53$ & 3.3 & 6.5 & 0.6 & $2,5,10,20,50,100$ \\
\hline NGC 1483 & $73 \times 56$ & 3.3 & 5.8 & 0.5 & $2,5,10,20,50,100$ \\
\hline ESO 201-G23 & $77 \times 52$ & 3.3 & 4.8 & 0.4 & $1,2,5,10$ \\
\hline ESO 249-G32 & $50 \times 21$ & 3.3 & 4.8 & 1.7 & $5,10,20,50,100,200$ \\
\hline APMUKS B0355-4643 & $50 \times 21$ & 3.3 & 4.8 & 1.7 & $5,10,20,50,100,200$ \\
\hline NGC 1448 & $157 \times 103$ & 6.6 & 1.7 & 0.1 & $0.5,1,2,5,10,20,50,100,200$ \\
\hline ESO 250-G5 & $149 \times 98$ & 3.3 & 4.9 & 0.1 & $0.5,1,2,5,10$ \\
\hline ESO 373-G7 & $82 \times 55$ & 3.3 & 8.5 & 0.7 & $2,5,10,20,50,100,200$ \\
\hline ESO $373-G 20$ & $89 \times 51$ & 3.3 & 4.1 & 0.3 & $1,2,5,10,20$ \\
\hline UGCA 168 & $82 \times 55$ & 3.3 & 8.5 & 0.7 & $2,5,10,20,50,100,200$ \\
\hline ESO 434-G41 & $82 \times 55$ & 3.3 & 10.0 & 0.8 & $2,5,10,20,50,100$ \\
\hline UGCA 182 & $93 \times 51$ & 3.3 & 4.1 & 0.3 & $1,2,5,10,20,50,100$ \\
\hline ESO 373-G6 & $102 \times 81$ & 3.3 & 3.4 & 0.2 & $1,2,5,10,20$ \\
\hline ESO 434-G19 & $99 \times 49$ & 3.3 & 4.7 & 0.5 & $2,5,10,20$ \\
\hline ESO 434-G17 & $87 \times 55$ & 3.3 & 7.0 & 0.5 & $2,5,10,20$ \\
\hline NGC $2997^{b}$ & $36 \times 29$ & 6.6 & 0.5 & 0.35 & $1,2,5,10,20,50,100$ \\
\hline UGCA 177 & $82 \times 55$ & 3.3 & 9.6 & 0.8 & $2,5,10,20,50,100$ \\
\hline IC 2507 & $82 \times 55$ & 3.3 & 8.0 & 0.6 & $2,5,10,20,50,100$ \\
\hline UGCA 180 & $82 \times 55$ & 3.3 & 8.0 & 0.6 & $2,5,10,20,50,100$ \\
\hline APMUKS B1237-0648 & $155 \times 124$ & 3.3 & 4.1 & 0.08 & $0.5,1,2,5$ \\
\hline UGCA 289 & $464 \times 349$ & 6.6 & 5.7 & 0.03 & $0.1,0.2,0.5,1,2,5,10$ \\
\hline NGC 4487 & $160 \times 125$ & 3.3 & 4.4 & 0.08 & $0.5,1,2,5,10,20,50$ \\
\hline NGC 4504 & $162 \times 121$ & 3.3 & 3.7 & 0.07 & $0.5,1,2,5,10,20,50$ \\
\hline NGC 4597 & $158 \times 125$ & 3.3 & 4.2 & 0.08 & $0.5,1,2,5,10,20,50,100$ \\
\hline [KKS2000] 30 & $157 \times 121$ & 3.3 & 4.2 & 0.08 & $0.5,1,2,5$ \\
\hline LCRSB1223-0616 & $154 \times 125$ & 3.3 & 4.5 & 0.08 & $0.5,1,2,5,10$ \\
\hline LCRSB1223-0612 & $154 \times 125$ & 3.3 & 4.5 & 0.08 & $0.5,1,2,5,10$ \\
\hline UGCA 286 & $157 \times 125$ & 3.3 & 4.4 & 0.08 & $0.5,1,2,5,10,20,50$ \\
\hline UGCA 295 & $155 \times 122$ & 3.3 & 4.3 & 0.08 & $0.5,1,2,5,10,20$ \\
\hline APMUKS B1224-0437 & $156 \times 125$ & 3.3 & 4.2 & 0.08 & $0.5,1,2,5$ \\
\hline DDO 142 & $155 \times 126$ & 3.3 & 4.6 & 0.09 & $0.5,1,2,5,10,20$ \\
\hline DDO 146 & $73 \times 53$ & 5.2 & 1.5 & 0.2 & $0.5,1,2,5,10,20,50$ \\
\hline APMUKS B2332-3729 & $123 \times 47$ & 3.3 & 4.1 & 0.3 & $1,2,5$ \\
\hline ESO 348-G9 & $90 \times 60$ & 3.3 & 3.3 & 0.2 & $0.5,1,2,5,10,20$ \\
\hline NGC 7713 & $111 \times 48$ & 3.3 & 5.0 & 0.3 & $1,2,5,10,20,50,100,200$ \\
\hline IC 5332 & $97 \times 61$ & 3.3 & 3.4 & 0.2 & $0.5,1,2,5,10,20,50$ \\
\hline ESO 347-G17 & $112 \times 50$ & 3.3 & 5.6 & 0.4 & $1,2,5,10,20,50$ \\
\hline NGC 5068 & $680 \times 72$ & 13.2 & 3.6 & 0.1 & $0.5,1,2,5,10,20,50$ \\
\hline UGCA 320 & $25 \times 18$ & 2.6 & 1.1 & 0.7 & $2,5,10,20,50,100,200,500$ \\
\hline SGC 1257-1909 & $177 \times 43$ & 3.3 & 6.9 & 0.3 & $1,2,5,10,20$ \\
\hline MCG-3-34-2 & $208 \times 42$ & 3.3 & 5.3 & 0.2 & $0.5,1,2,5,10$ \\
\hline
\end{tabular}

Notes.

${ }^{\text {a }}$ Corresponding to Figures 19-25.

${ }^{\mathrm{b}}$ Data taken from Hess et al. (2009). 

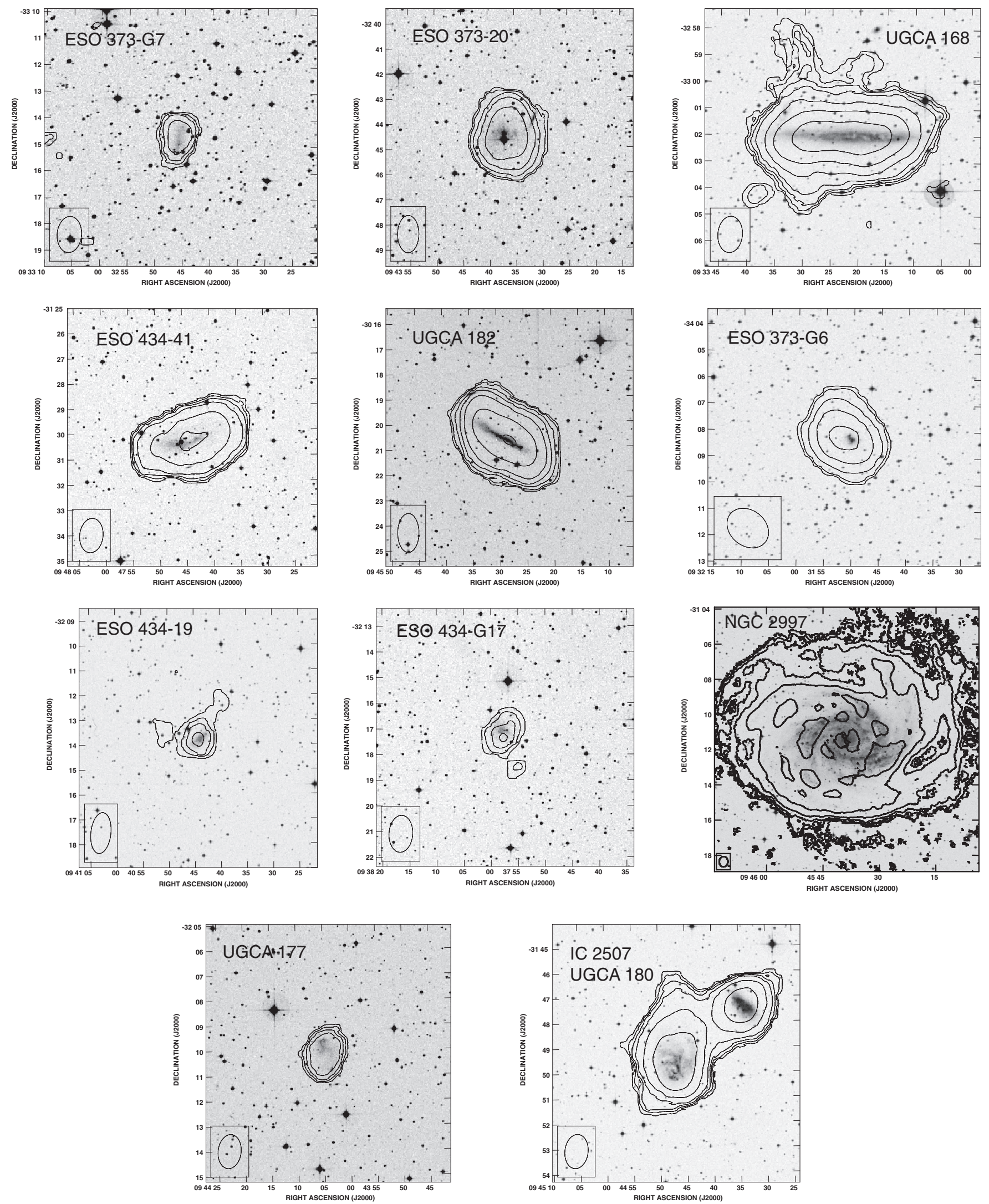

Figure 22. Same as Figure 19, but for LGG 180 group galaxies. 

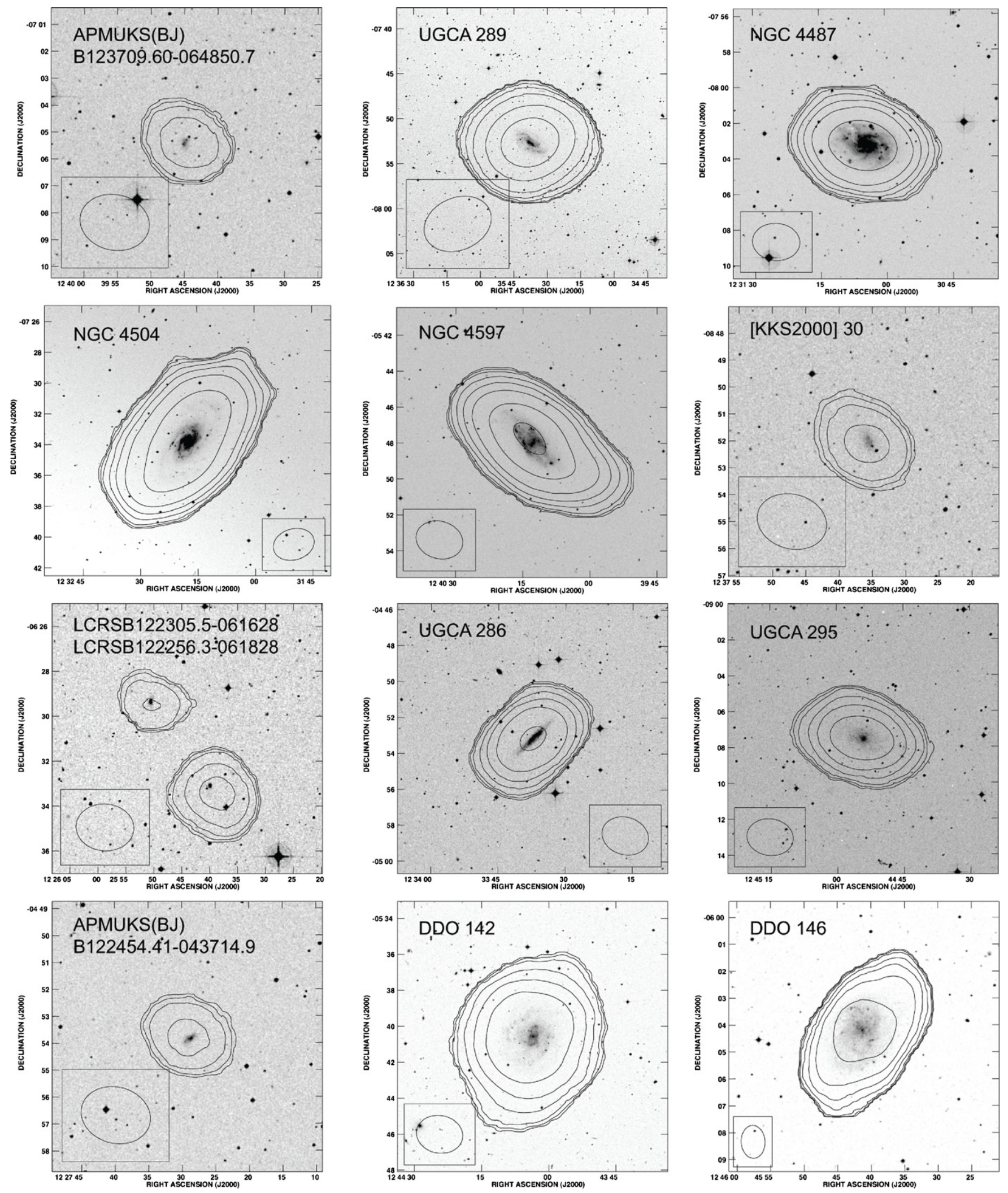

Figure 23. Same as Figure 19, but for LGG 293 group galaxies. 
Table 5

Interferometer Data for Background Galaxies

\begin{tabular}{|c|c|c|c|c|c|}
\hline \multirow[t]{2}{*}{ Galaxy } & \multirow{2}{*}{$\begin{array}{c}\text { Beam Size } \\
(\operatorname{arcsec})\end{array}$} & \multirow{2}{*}{$\begin{array}{c}\text { Channel Width } \\
\left(\mathrm{km} \mathrm{s}^{-1}\right)\end{array}$} & \multicolumn{2}{|c|}{ Noise } & \multirow{2}{*}{$\begin{array}{c}\text { Contour Levels }^{\mathrm{a}} \\
\left(10^{19} \mathrm{~cm}^{-2}\right)\end{array}$} \\
\hline & & & $\left(\mathrm{mJy}\right.$ beam $\left.^{-1}\right)$ & $\left(10^{19} \mathrm{~cm}^{-2}\right)$ & \\
\hline ESO 201-G2 & $75 \times 53$ & 3.3 & 6.5 & 0.6 & $2,5,10$ \\
\hline IC 1986 & $102 \times 49$ & 13.2 & 4.5 & 1.3 & $5,10,20,50$ \\
\hline LSBG F249-040 & $178 \times 123$ & 3.3 & 5.0 & 0.08 & $0.5,1,2,5,10$ \\
\hline IC 2009 & $75 \times 53$ & 3.3 & 6.1 & 0.6 & $2,5,10,20$ \\
\hline APMUKS B1237-0724 & $157 \times 125$ & 3.3 & 4.1 & 0.08 & $0.5,1,2,5,10$ \\
\hline APMUKS B1236-0417 & $197 \times 116$ & 3.3 & 6.3 & 0.1 & $0.5,1,2,5,10$ \\
\hline NGC 4602 & $204 \times 116$ & 3.3 & 6.6 & 0.1 & $0.5,1,2,5,10,20$ \\
\hline FGC 1496 & $153 \times 125$ & 3.3 & 4.3 & 0.08 & $0.5,1,2,5,10,20$ \\
\hline NGC 4626 & $209 \times 115$ & 3.3 & 6.1 & 0.09 & $0.5,1,2,5,10,20$ \\
\hline LGG 293-HI-10 & $212 \times 114$ & 3.3 & 6.6 & 0.1 & $0.5,1,2,5,10$ \\
\hline NGC 4433 & $202 \times 114$ & 3.3 & 6.0 & 0.09 & $0.5,1,2,5$ \\
\hline ESO 347-G29 & $63 \times 30$ & 6.6 & 1.6 & 0.6 & $2,5,10,20,50,100$ \\
\hline NGC 7764 & $87 \times 52$ & 3.3 & 6.0 & 0.5 & $2,5,10,20,50$ \\
\hline APMUKS B2341-3703 & $101 \times 55$ & 3.3 & 3.3 & 0.2 & $0.5,1,2,5,10$ \\
\hline APMUKS B2347-3649 & $109 \times 53$ & 3.3 & 3.6 & 0.2 & $0.5,1,2,5,10$ \\
\hline ESO 408-G12 & $123 \times 45$ & 3.3 & 5.3 & 0.3 & $1,2,3$ \\
\hline ESO $347-G 23$ & $109 \times 49$ & 3.3 & 4.4 & 0.3 & $1,2,5,10$ \\
\hline NGC 7713A & $124 \times 48$ & 3.3 & 5.1 & 0.3 & $1,2,5,10,20$ \\
\hline 2MASX J1314-2203 & $155 \times 43$ & 3.3 & 5.6 & 0.3 & $1,2,5,10$ \\
\hline UGCA 356 & $158 \times 42$ & 3.3 & 7.0 & 0.4 & $1,2,5,10,20$ \\
\hline DDO 164 & $225 \times 39$ & 3.3 & 7.0 & 0.3 & $1,2,5,10,20$ \\
\hline MCG-3-34-67 & $192 \times 46$ & 3.3 & 5.8 & 0.2 & $0.5,1,2,5,10,20$ \\
\hline NGC 5170 & $170 \times 49$ & 3.3 & 8.0 & 0.3 & $1,2,5,10,20,50,100$ \\
\hline LEDA 083827 & $205 \times 44$ & 3.3 & 5.3 & 0.2 & $0.5,1,2,5,10$ \\
\hline ESO 576-G25 & $152 \times 47$ & 3.3 & 7.6 & 0.4 & $1,2,5,10$ \\
\hline ESO 575-G61 & $123 \times 50$ & 6.6 & 2.5 & 0.3 & $1,2,5,10$ \\
\hline NGC 5054 & $193 \times 46$ & 3.3 & 7.6 & 0.3 & $1,2,5,10,20$ \\
\hline UGCA 348 & $132 \times 50$ & 3.3 & 6.3 & 0.3 & $1,2,5,10,20$ \\
\hline NGC 5134 & $143 \times 48$ & 3.3 & 7.6 & 0.4 & $1,2,5,10,20$ \\
\hline NGC 5084 & $222 \times 72$ & 6.6 & 4.4 & 0.2 & $0.5,1,2,5,10,20,50$ \\
\hline 2MASX J1324-2015 & $165 \times 43$ & 3.3 & 5.0 & 0.3 & $1,2,5$ \\
\hline ESO 576-G42 & $173 \times 42$ & 3.3 & 6.5 & 0.3 & $1,2,5,10$ \\
\hline UGCA 353 & $165 \times 45$ & 3.3 & 7.2 & 0.4 & $1,2,5,10,20,50$ \\
\hline ESO 576-G40 & $154 \times 44$ & 3.3 & 6.2 & 0.3 & $1,2,5,10,20,50,100$ \\
\hline IC 863 & $197 \times 43$ & 3.3 & 5.0 & 0.2 & $0.5,1,2,5,10$ \\
\hline GALEX 2698124594575839357 & $197 \times 43$ & 3.3 & 5.0 & 0.2 & $0.5,1,2,5,10$ \\
\hline SGC 1316-1722 & $212 \times 41$ & 3.3 & 7.1 & 0.3 & $1,2,5,10$ \\
\hline MCG-3-34-4 & $296 \times 37$ & 3.3 & 7.8 & 0.3 & $1,2,5,10,20$ \\
\hline ESO 575-G53 & $143 \times 46$ & 3.3 & 6.7 & 0.4 & $1,2,5,10,20$ \\
\hline SGC 1317-1702 & $189 \times 44$ & 3.3 & 6.6 & 0.3 & $1,2,5,10,20$ \\
\hline MCG-3-34-41 & $202 \times 44$ & 3.3 & 6.9 & 0.3 & $1,2,5,10,20$ \\
\hline
\end{tabular}

Note. ${ }^{\text {a }}$ Corresponding to Figures 26-29.

interferometer observations are listed in Tables 4 and 5. For the remainder of this paper, we will limit our discussion to those galaxies belonging to the targeted groups.

The derived properties of the group galaxies are listed in Table 6. As shown in Figures 31-33, these galaxies span a wide range of $\mathrm{HI}_{\mathrm{I}}$ mass with $M_{\mathrm{HI}} \sim 10^{7}-10^{10} M_{\odot}$ and luminosity, from $M_{B}=-12.4-21.3 \mathrm{mag}\left(L_{B}=1.4 \times 10^{7}-5.1 \times 10^{10} L_{\odot}\right)$. These figures clearly show that most of the galaxies detected in $\mathrm{HI}_{\mathrm{I}}$ in these groups span the full range of luminosities and $M_{\mathrm{HI}}$ from the median of Local Group H I-rich dwarfs right through to the median of typical spiral galaxies. The galaxies also have $\mathrm{H}$ I-mass-to-light ratios that are generally consistent with those of late-type spiral galaxies or Local Group H I-rich dwarf irregulars with $M_{\mathrm{H}_{\mathrm{I}}} / L_{B} \sim 0.1-5 M_{\odot} / L_{\odot}$ with few exceptions. Those exceptions are two extremely gas-rich galaxies: ESO $348-\mathrm{G} 9$ and ESO $373-\mathrm{G} 7$ with $M_{\mathrm{HI}} / L_{B}$ of 9.27 and $26 M_{\odot} / L_{\odot}$, respectively, and one relatively gas-poor galaxy, ESO 250-G5, a lenticular with $M_{\mathrm{HI}} / L_{B}$ of $0.01 M_{\odot} / L_{\odot}$ that has a remarkably low $M_{\mathrm{HI}} \sim 10^{7} M_{\odot}$.

\section{Hi MASS FUNCTION}

Our primary goal in this paper is to determine how the mass function of galaxies in the low-density group environment compares to that in the field in general and in denser environments. We begin by examining the HIMF for loose groups.

To construct the HIMF, we followed the same bivariate stepwise maximum likelihood method described by Zwaan et al. (2003) that accounts for the survey completeness as a function of linewidth and integrated flux. For the HIMF we placed each galaxy in 0.3 dex wide $M_{\mathrm{HI}}$ bins with a weight based on the scaled HIPASS completeness function for that galaxy (described in Paper I). To convert to a volume density of galaxies, we took the total volume of the survey assuming that each loose 
Table 6

Derived Properties of Group Galaxies

\begin{tabular}{|c|c|c|c|c|c|c|}
\hline Galaxy & $\begin{array}{c}\text { Distance }^{\mathrm{a}} \\
(\mathrm{Mpc})\end{array}$ & $\begin{array}{c}M_{\mathrm{H}_{\mathrm{I}}} \\
\left(10^{8} M_{\odot}\right)\end{array}$ & $\begin{array}{c}\text { Inclination }^{\mathrm{b}} \\
\left({ }^{\circ}\right)\end{array}$ & $\begin{array}{c}V_{\text {rot }^{\mathrm{c}}} \\
\left(\mathrm{km} \mathrm{s}^{-1}\right)\end{array}$ & $\begin{array}{c}M_{B}{ }^{\mathrm{d}} \\
(\mathrm{mag})\end{array}$ & $\begin{array}{c}M_{\mathrm{HI}_{\mathrm{I}}} / L_{B} \\
\left(M_{\odot} / L_{\odot}\right) \\
\end{array}$ \\
\hline NGC 1311 & 10.9 & 3.93 & 90 & 45 & -16.9 & 0.45 \\
\hline IC 1959 & 10.9 & 7.91 & 90 & 65 & -17.0 & 0.78 \\
\hline ESO 200-G45 & 10.9 & 1.01 & 37 & 36 & -13.9 & 1.71 \\
\hline IC 1914 & 10.9 & 12.42 & 43 & 141 & -16.8 & 1.55 \\
\hline LSBG F200-023 & 10.9 & 0.62 & 45 & 53 & -12.4 & 4.32 \\
\hline IC 1954 & 10.9 & 5.66 & 69 & 111 & -18.2 & 0.20 \\
\hline IC 1896 & 10.9 & 0.76 & 76 & 52 & -15.2 & 0.39 \\
\hline IC 1933 & 10.9 & 6.90 & 58 & 108 & -17.4 & 0.50 \\
\hline NGC 1249 & 10.9 & 27.79 & 69 & 115 & -18.1 & 1.07 \\
\hline AM 0311-492 & 10.9 & 0.45 & 52 & 34 & -12.9 & 2.02 \\
\hline APMUKS B0403-4939 & 13.8 & 0.40 & 71 & 17 & -12.5 & 2.70 \\
\hline ESO 249-G36 & 13.8 & 6.92 & 42 & 51 & -15.4 & 2.97 \\
\hline IC 2000 & 13.8 & 14.34 & 90 & 128 & -17.7 & 0.74 \\
\hline IC 2004 & 13.8 & 0.58 & 44 & 77 & -15.7 & 0.19 \\
\hline AM $0358-465$ & 13.8 & 1.66 & 43 & 53 & -15.8 & 0.49 \\
\hline ESO 249-G35 & 13.8 & 2.38 & 90 & 55 & -14.5 & 2.34 \\
\hline NGC 1433 & 13.8 & 13.98 & 67 & 88 & -19.9 & 0.09 \\
\hline 6dF J0351-4635 & 13.8 & 0.36 & 17 & 82 & -14.8 & 0.27 \\
\hline ESO 201-G14 & 13.8 & 3.69 & 90 & 73 & -16.6 & 0.52 \\
\hline NGC 1493 & 13.8 & 18.52 & 23 & 132 & -18.9 & 0.31 \\
\hline NGC 1494 & 13.8 & 12.99 & 69 & 86 & -18.5 & 0.32 \\
\hline NGC 1483 & 13.8 & 8.76 & 37 & 112 & -17.5 & 0.55 \\
\hline ESO 201-G23 & 13.8 & 1.12 & 62 & 40 & -14.2 & 1.55 \\
\hline ESO 249-G32 & 13.8 & 2.38 & 90 & 30 & -14.4 & 2.57 \\
\hline APMUKS B0355-4643 & 13.8 & 1.17 & 60 & 45 & -13.7 & 2.40 \\
\hline NGC 1448 & 13.8 & 9.26 & 86 & 190 & -19.4 & 0.11 \\
\hline ESO 250-G5 & 13.8 & 0.09 & 60 & 21 & -16.6 & 0.01 \\
\hline ESO 373-G7 & 14.8 & 41.25 & 66 & 113 & -15.0 & 26.00 \\
\hline ESO 373-G20 & 14.8 & 4.86 & 47 & 42 & -15.3 & 2.48 \\
\hline UGCA 168 & 14.8 & 32.00 & 79 & 105 & -18.7 & 0.66 \\
\hline ESO 434-G41 & 14.8 & 9.51 & 90 & 48 & -16.5 & 1.56 \\
\hline UGCA 182 & 14.8 & 11.73 & 90 & 62 & -16.7 & 1.63 \\
\hline ESO 373-G6 & 14.8 & 1.91 & 50 & 53 & -15.2 & 0.99 \\
\hline ESO 434-G19 & 14.8 & 2.64 & 88 & 56 & -16.2 & 0.57 \\
\hline ESO 434-G17 & 14.8 & 2.48 & 55 & 51 & -15.5 & 1.01 \\
\hline NGC 2997 & 14.8 & 98.84 & 32 & 233 & -21.3 & 0.19 \\
\hline UGCA 177 & 14.8 & 3.62 & 15 & 162 & -16.2 & 0.79 \\
\hline IC 2507 & 14.8 & 10.75 & 73 & 70 & -18.0 & 0.43 \\
\hline UGCA 180 & 14.8 & 17.16 & 33 & 120 & -17.9 & 0.75 \\
\hline APMUKS B1237-0648 & 11.1 & 0.76 & 90 & 37 & -13.9 & 1.32 \\
\hline UGCA 289 & 11.1 & 7.82 & 90 & 76 & -15.4 & 3.33 \\
\hline NGC 4487 & 11.1 & 10.64 & 46 & 132 & -18.5 & 0.27 \\
\hline NGC 4504 & 11.1 & 27.30 & 50 & 143 & -18.1 & 0.98 \\
\hline NGC 4597 & 11.1 & 17.85 & 90 & 80 & -17.4 & 1.22 \\
\hline [KKS2000] 30 & 11.1 & 0.49 & 66 & 20 & -13.1 & 1.89 \\
\hline LCRSB1223-0616 & 11.1 & 0.87 & 53 & 28 & -12.6 & 5.18 \\
\hline LCRSB1223-0612 & 11.1 & 0.38 & 58 & 23 & -12.8 & 1.87 \\
\hline UGCA 286 & 11.1 & 5.84 & 90 & 64 & -15.2 & 3.13 \\
\hline UGCA 295 & 11.1 & 2.41 & 22 & 133 & -16.0 & 0.63 \\
\hline APMUKS B1224-0437 & 11.1 & 0.64 & 77 & 25 & -13.5 & 1.57 \\
\hline DDO 142 & 11.1 & 11.05 & 27 & 130 & -17.4 & 0.81 \\
\hline DDO 146 & 11.1 & 4.74 & 52 & 88 & -17.3 & 0.35 \\
\hline APMUKS B2332-3729 & 8.6 & 0.19 & 62 & 22 & -13.1 & 0.68 \\
\hline ESO 348-G9 & 8.6 & 2.36 & 90 & 43 & -13.0 & 9.27 \\
\hline NGC 7713 & 8.6 & 10.89 & 66 & 102 & -18.2 & 0.35 \\
\hline IC 5332 & 8.6 & 29.34 & 18 & 164 & -18.4 & 0.79 \\
\hline ESO 347-G17 & 8.6 & 1.62 & 90 & 38 & -14.8 & 1.21 \\
\hline NGC 5068 & 9.1 & 26.09 & 27 & 105 & -19.6 & 0.23 \\
\hline UGCA 320 & 9.1 & 20.97 & 90 & 54 & -16.6 & 2.96 \\
\hline SGC 1257-1909 & 9.1 & 0.88 & 67 & 23 & -13.7 & 1.85 \\
\hline MCG-3-34-2 & 9.1 & 0.21 & 54 & 27 & -15.3 & 0.10 \\
\hline
\end{tabular}

Notes.

a Taken from Paper I.

b Taken from HyperLeda, where available, or calculated in same fashion using data from NED.

c Calculated from $W_{20}$ using the method described in Meyer et al. (2008).

${ }^{\mathrm{d}}$ Calculated from apparent $B$ magnitude from HyperLeda or $b_{J}$ magnitude from NED. Corrected for external extinction using Schlegel et al. (1998), no internal extinction correction applied. 

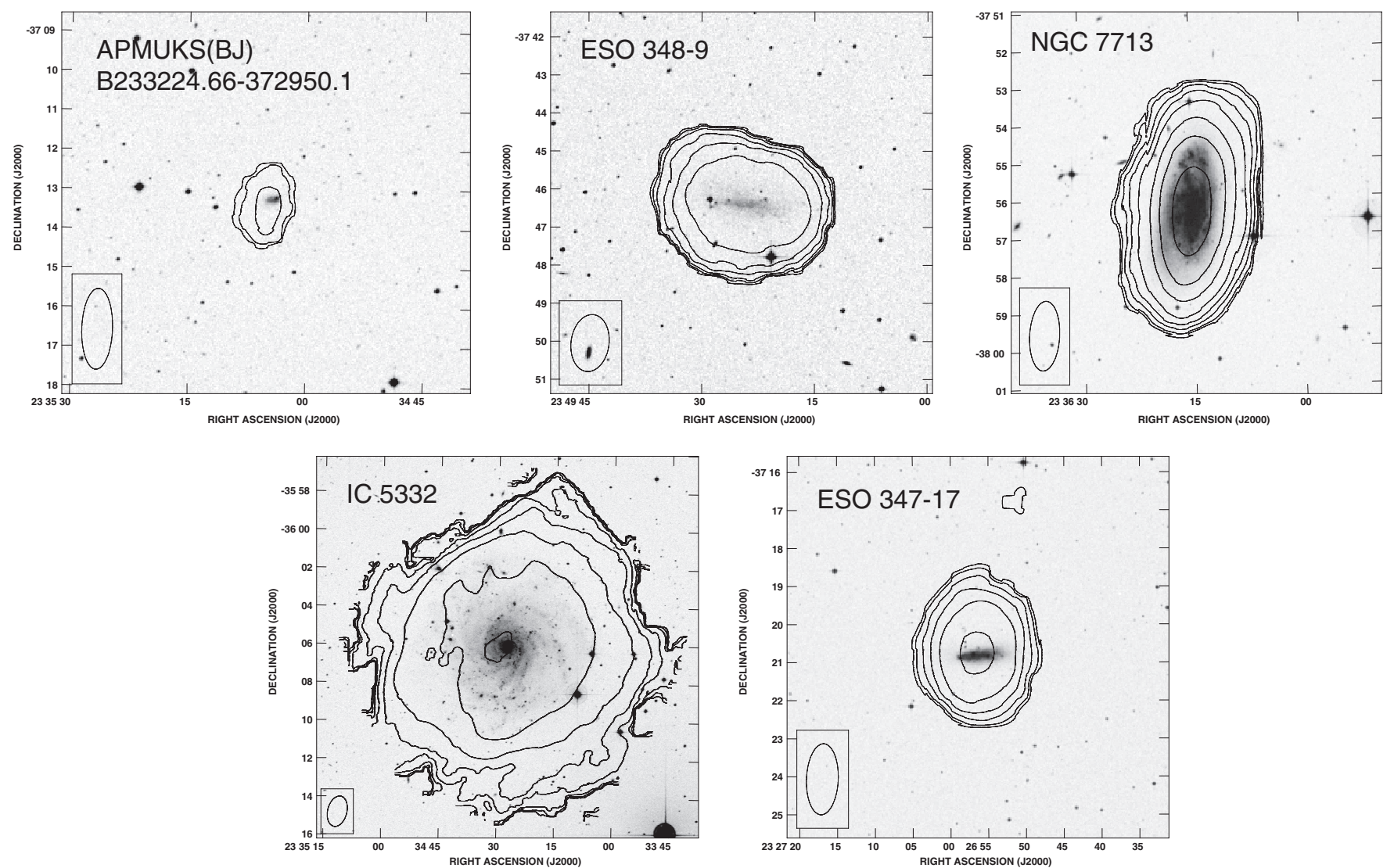

Figure 24. Same as Figure 19, but for LGG 478 group galaxies.

group covered the entire survey area and had a depth equal to its diameter for a total survey volume of $7.81 \mathrm{Mpc}^{3}$ for the six groups. The result is shown at the top of Figure 33. For comparison, we also show the HIMF for those Local Group galaxies that have been detected in $\mathrm{H}$ I, using data from Mateo (1998) and Grcevich \& Putman (2009) for the dwarf galaxies, Staveley-Smith et al. (2003) for the Large Magellanic Cloud (LMC), Stanimirovic et al. (1999) for the Small Magellanic Cloud (SMC), and van den Bergh (2000) for the Milky Way, M 31, and M 33. For this HIMF, we assumed that the volume of the Local Group was the same as the average loose group. Aside from the two lowest $M_{\mathrm{HI}}$ bins, where the completeness is the most uncertain, there is very good agreement in the slope and normalization of the HIMF for the Local Group and our sample of loose groups. For comparison, we have also shown the HIMF from the HIPASS galaxies derived by Zwaan et al. (2005) as described by a Schechter function of the form

$$
\Theta(M)=\Theta^{\star} \ln (10)\left(\frac{M_{\mathrm{HI}}}{M^{\star}}\right)^{\alpha+1} \exp \left(-\frac{M_{\mathrm{HI}}}{M^{\star}}\right),
$$

where $M^{\star}$ is the mass where the function transitions to a lowmass power law with slope $\alpha$ and $\Theta^{\star}$ is the normalization. For HIPASS, $\log M_{\mathrm{H}_{\mathrm{I}}}{ }^{\star}=9.8, \Theta^{\star}=0.006 \mathrm{Mpc}^{-3} \mathrm{dex}^{-1}$, and $\alpha=-1.37$ (Zwaan et al. 2005). We also show an identical HIMF with a faint-end slope of $\alpha=-1.0$. In this figure, these two HIMFs have been renormalized to approximately match the loose group and Local Group mass functions. While not shown on the figure, the recent HIMF from ALFALFA (Martin et al. 2010) is based on over twice as many galaxies and has a wider mass range than the HIPASS HIMF (Zwaan et al. 2005), although most of its low-mass sources are in the high-density Virgo Cluster. Nevertheless, the resulting fit is not dramatically different with $\log M_{\mathrm{HI}^{\star}}{ }^{\star}=9.96, \Theta^{\star}=0.0048 \mathrm{Mpc}^{-3} \mathrm{dex}^{-1}$, and $\alpha=-1.33$.

There have been many recent measurements of how the low-mass slope, $\alpha$, of the HIMF varies with the local galaxy density. Zwaan et al. (2005) calculated the HIMF for HIPASS galaxies in different density regions and found that as the local density decreased, the slope became flatter. Zwaan et al. (2005), however, used the HIPASS catalog to define the local galaxy density, so H I observations of galaxies in specific environments are needed to provide independent confirmation of these results. Such observations generally support the conclusions of Zwaan et al. (2005). Freeland et al. (2009) assembled an HIMF for five groups, four of which lack X-rays and are spiral-rich, and found a flat, $\alpha=-1.0$, HIMF. Kovač et al. (2005, 2009) found a similar flat slope, $\alpha=-1.07$, in the low-density Canes Venatici group, as did Verheijen et al. (2001) for the low-density Ursa Major cluster. In higher density groups, that contain more early-type galaxies and X-ray emission, the results are more varied. Kilborn et al. (2009) found a declining lowmass slope, $\alpha=0.0$, while Stierwalt et al. (2009) found a steep low-mass slope close to that found by Zwaan et al. (2005), $\alpha=-1.41$. In higher density clusters, the low- $M_{\mathrm{H}}$ slope also tends to be steeper (Gavazzi et al. 2005, 2006), however this may not hold in the centers of clusters where there is a lack of H I-rich galaxies (cf. Davies et al. 2004; Springob et al. 2005). In light of these past results, our HIMFs for the lowdensity environments of the Local Group and our sample of six analogous loose groups are consistent with a flattening HIMF in lower density environments. This behavior is mimicked by 

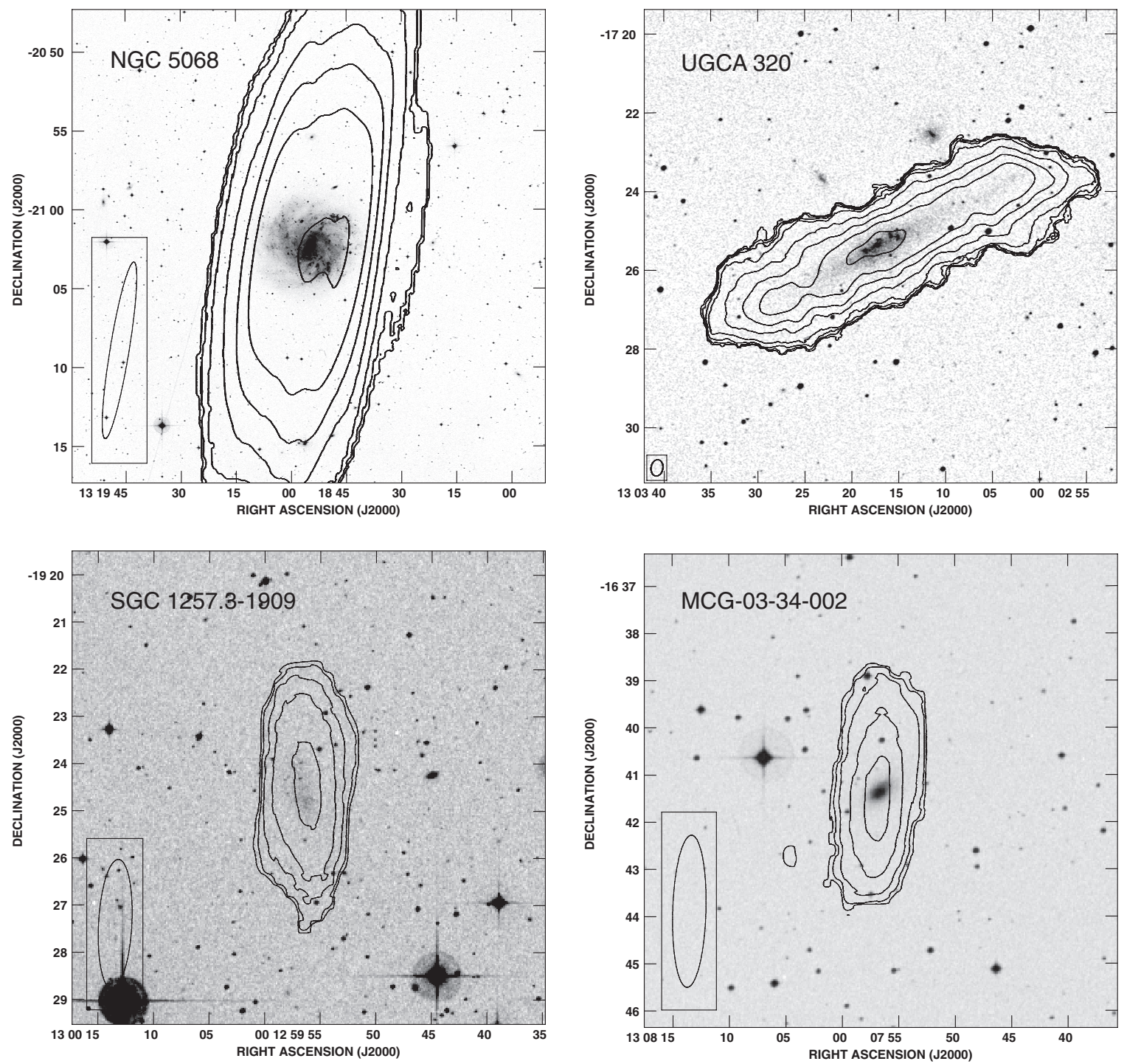

Figure 25. Same as Figure 19, but for HIPASS group galaxies.

the optical luminosity functions with flatter slopes found in lower density environments (Tully et al. 2002), although Croton et al. (2005) found little variation in the faint-end slope with environment.

Zwaan et al. (2005) provide two possible explanations for the flattening of the low-mass slope of the HIMF in low-density environments. The first is that since the star formation rate and the specific star formation rate are enhanced in lower density environments, then the $\mathrm{H}$ I in group galaxies will be consumed faster than in cluster galaxies, particularly at low $M_{\mathrm{H}_{\mathrm{I}}}$. Enhanced merging of low- $M_{\mathrm{H}}$ galaxies could also provide a surplus of high- $M_{\mathrm{H} \text { I }}$ galaxies in low-density environments. Both of these processes could lead to a flattening of the HIMF. In the higher density clusters and groups that contain a hot, dense IGM, ram pressure stripping, as is seen in the Virgo Cluster (Chung et al. 2009), could shift galaxies from higher $M_{\mathrm{HI}}$ to lower $M_{\mathrm{HI}}$, causing the HIMF to steepen in these environments. While the densities are low, two of our groups, LGG 93 and LGG 180, have $\mathrm{H}$ I deficiencies of $0.2 \pm 0.11$ and $0.1 \pm 0.05$ based on HIPASS data (Sengupta \& Balasubramanyam 2006). Even without an $\mathrm{X}$-ray bright IGM, it is possible that these groups could have a cool, dense IGM (e.g., Freeland \& Wilcots 2011) that could cause ram pressure stripping. Alternatively, stripping from tidal interactions could be occurring in at least these two groups, although neither form of stripping would explain the flat HIMF for our groups. The variations of $\alpha$ between groups and clusters of similar density may be due to other effects. For example, in order to sustain star formation beyond the next gigayear, we know that the Milky Way needs to accrete more gas (e.g., Peek 2009). There have been many processes proposed to halt the accretion of cold gas onto galaxies, and, hence, halt star formation. These include shock heating the accreting gas to the virial temperature of the halo (Cattaneo et al. 2006), or heating from active galactic nuclei (AGNs), supernovae-driven winds, and/or star formation (Hopkins et al. 2006). Finally, Zwaan et al. (2005) suggested that the flat slope of the HIMF in low density regions can be explained by the halo occupation model of Mo et al. (2004), such that the late-type galaxies that dominate groups also reside in halos with a flat low-mass slope. Simulations of galaxy formation are just starting to become sophisticated enough to predict how $M_{\mathrm{H}}$ varies with galaxy properties and environment (Duffy et al. 2011), so, in order to more directly compare with simulations, we construct the CVDF for loose groups. 

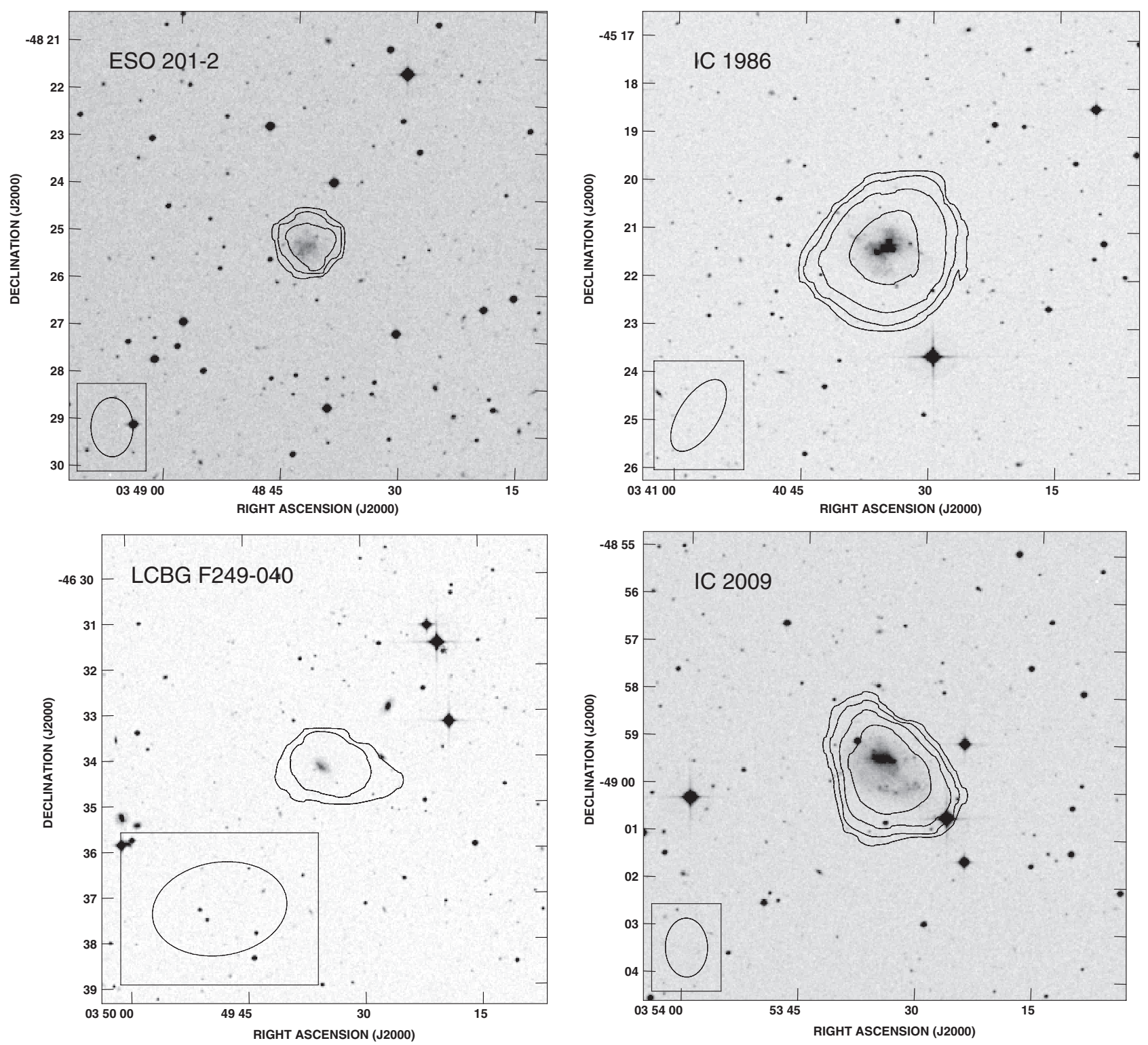

Figure 26. Same as Figure 19, but for galaxies behind LGG 106. The contour levels are listed in Table 5. 

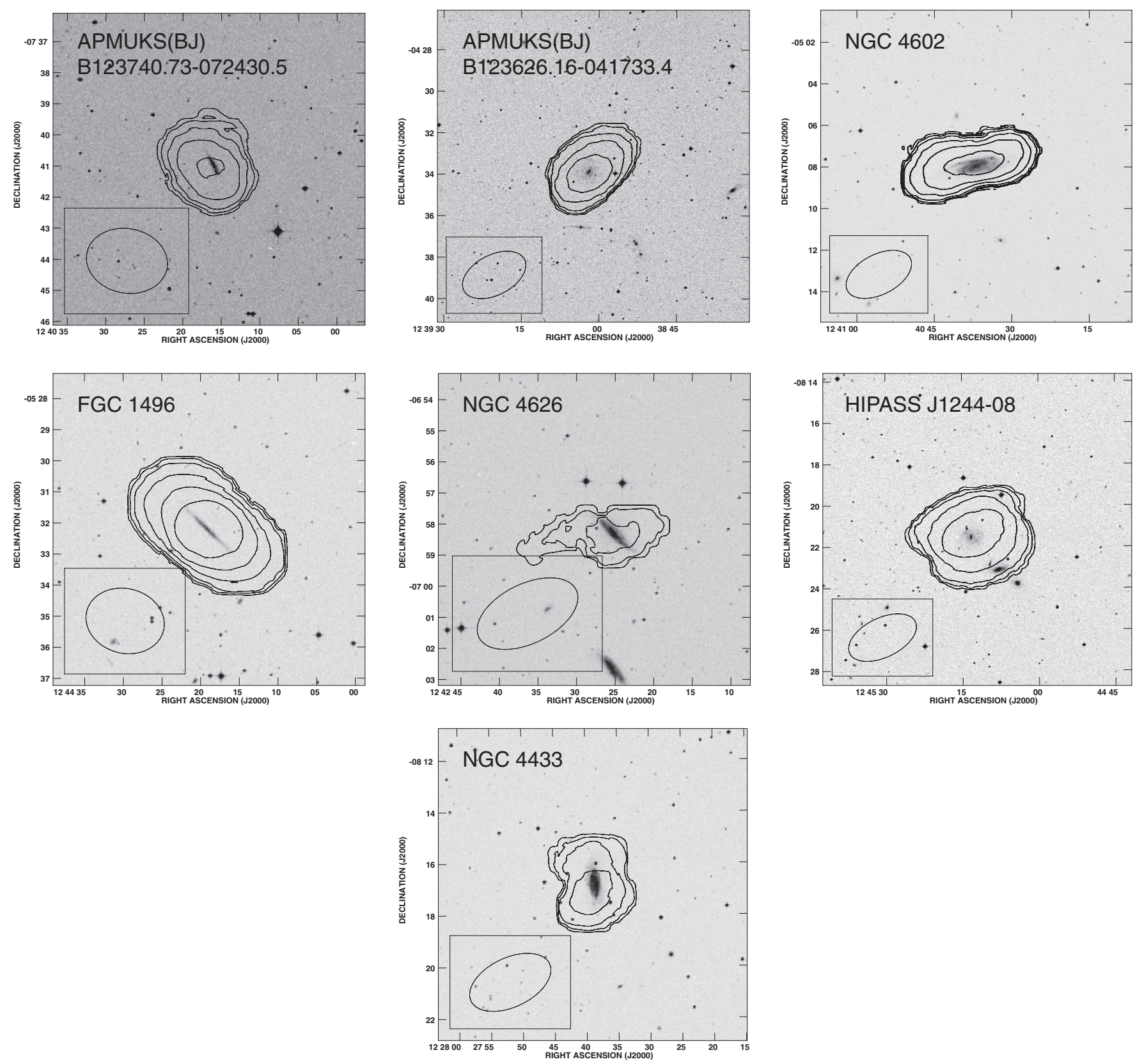

Figure 27. Same as Figure 26, but for galaxies behind LGG 293. 

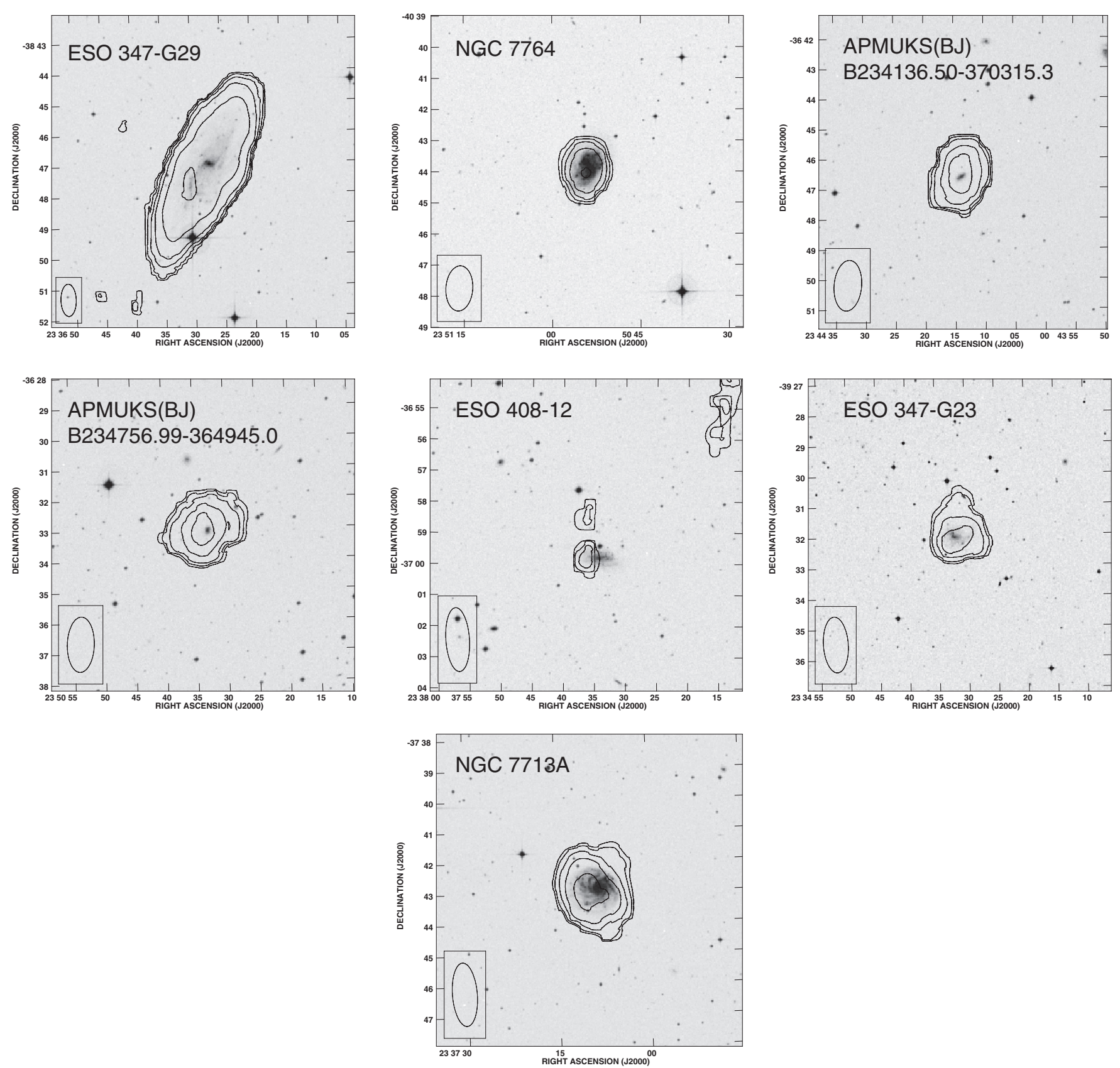

Figure 28. Same as Figure 26, but for galaxies behind LGG 478. 

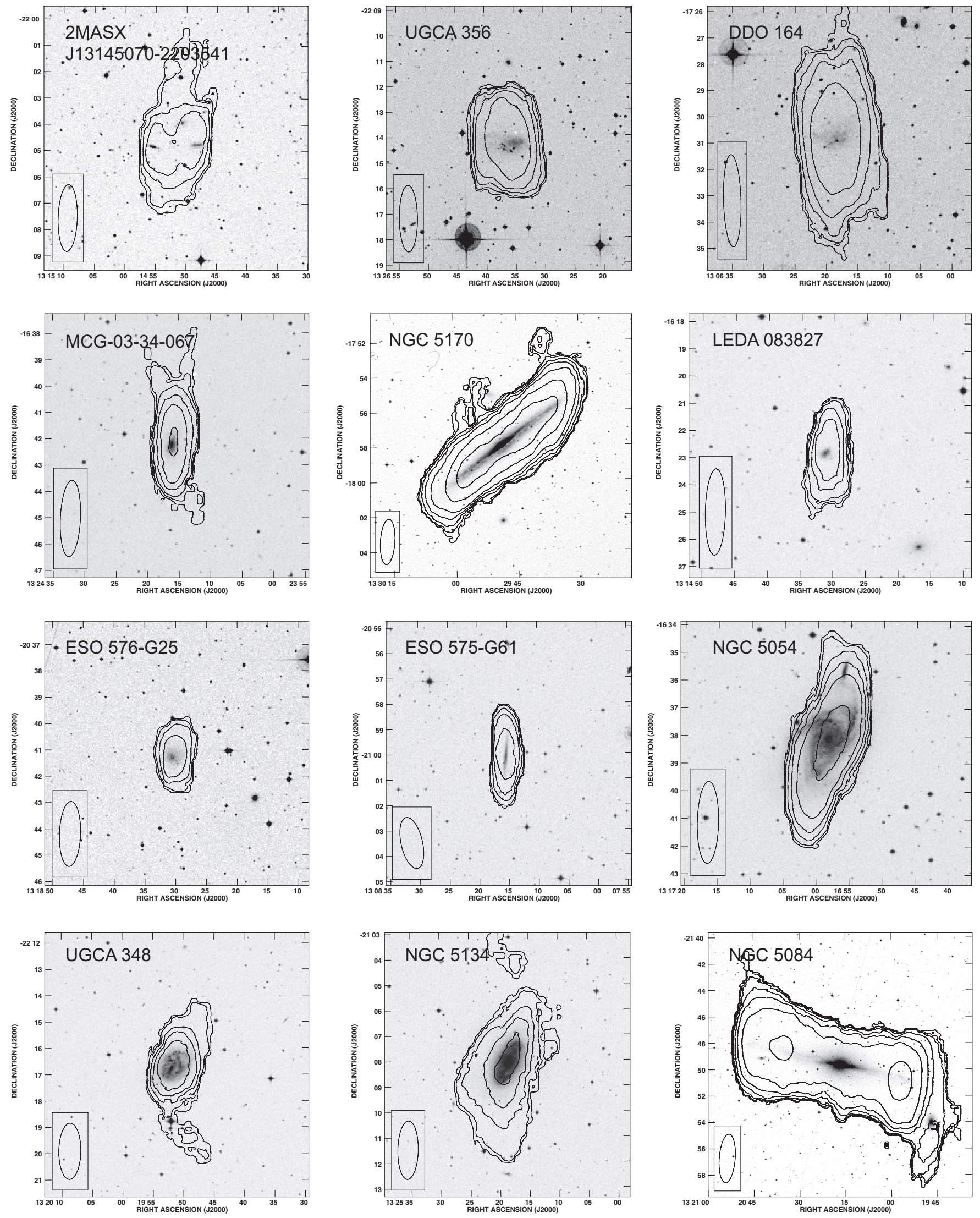

Figure 29. Same as Figure 26, but for galaxies behind the HIPASS group. 

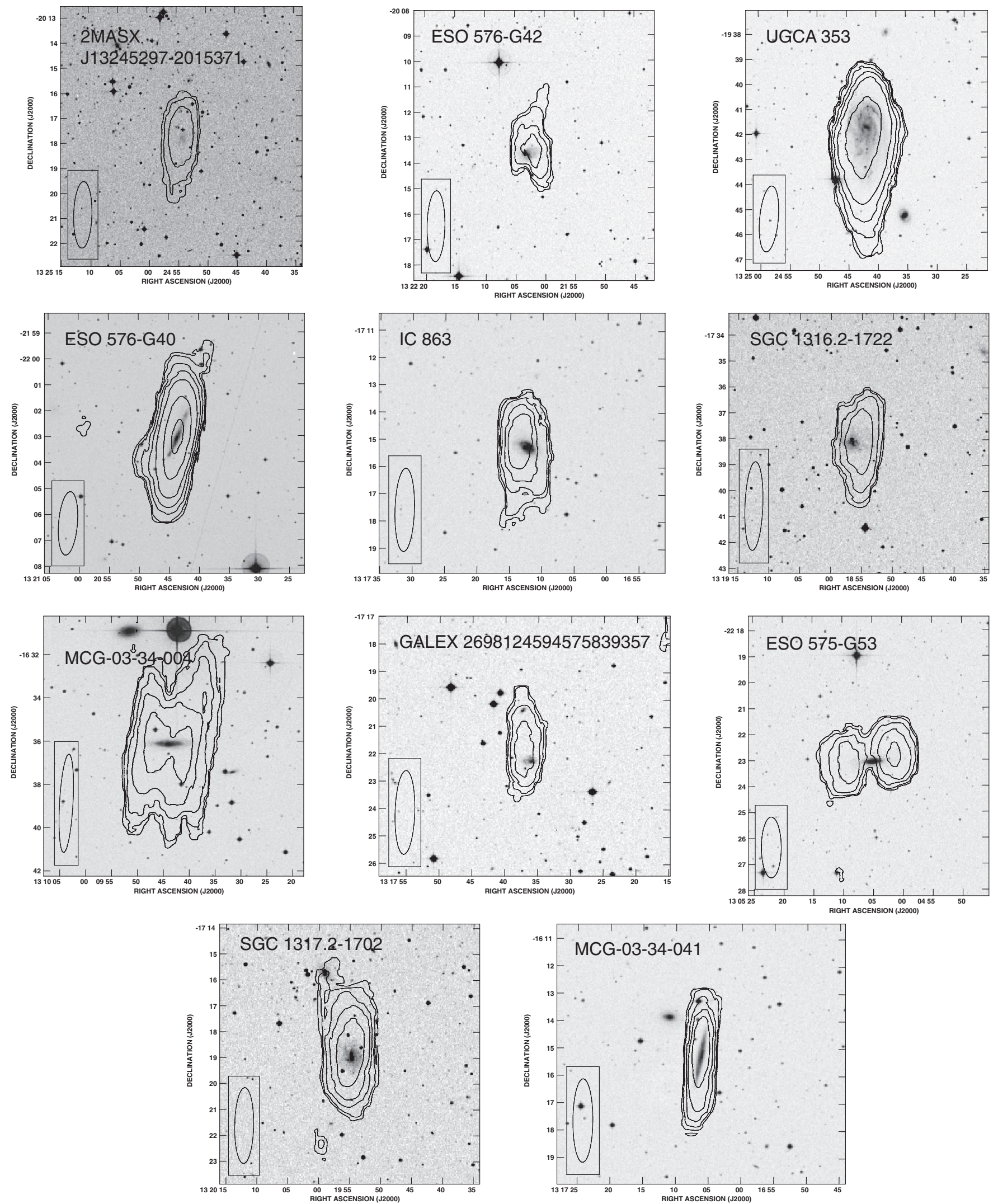

Figure 30. Same as Figure 26, but for the remaining galaxies behind the HIPASS group. 


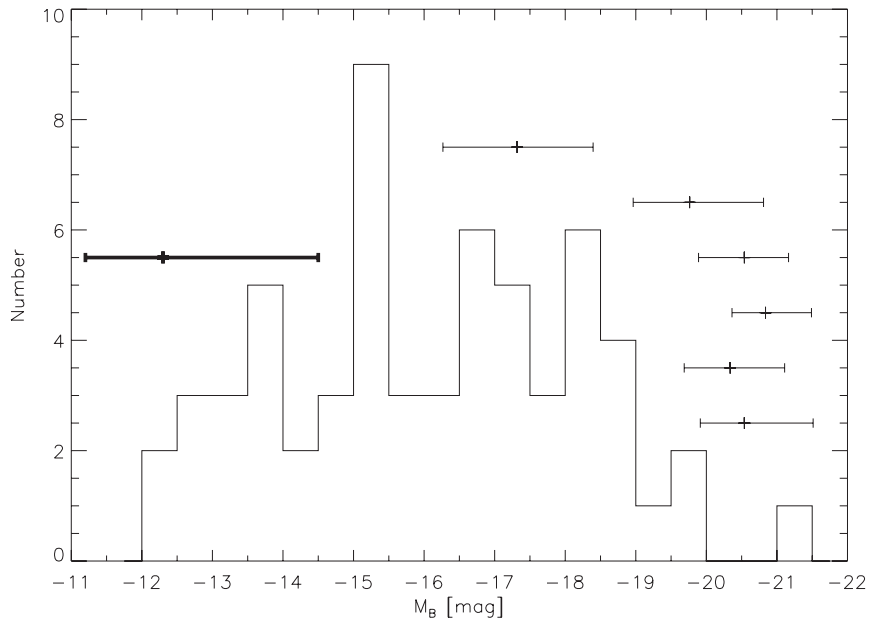

Figure 31. Histogram showing the distribution of absolute $B$-band magnitudes for the group galaxies. The error bars indicate the 25 th, 50 th, and 75 th percentile distribution of $M_{B}$ for UGC galaxies as reported by Roberts \& Haynes (1994) and corrected for $H_{0}=72 \mathrm{~km} \mathrm{~s}^{-1} \mathrm{Mpc}^{-1}$. From bottom to top they represent these values for $\mathrm{E} / \mathrm{S} 0, \mathrm{~S} 0 \mathrm{a} / \mathrm{Sa}, \mathrm{Sab} / \mathrm{Sb}, \mathrm{Sbc} / \mathrm{Sc}, \mathrm{Scd} / \mathrm{Sd}$, and $\mathrm{Sm} / \mathrm{Im}$ galaxies. The thick error bar indicates the same thing for Local Group dwarf galaxies with H I detections from Mateo (1998) and Kalirai et al. (2010). The group galaxies detected in $\mathrm{HI}_{\mathrm{I}}$ are preferentially lower luminosity galaxies compared with traditional galaxies on the Hubble sequence; our survey is primarily detecting dwarf galaxies.

\section{CIRCULAR VELOCITY DISTRIBUTION FUNCTION}

The CVDF uses the measured or inferred circular rotation velocity of a galaxy as a proxy for the mass of the dark matter halo within which the galaxy resides. $V_{\text {circ }}$ is a robust measure of the total dark matter mass in simulations and it can be compared with observations at least for those halos that host a luminous galaxy. An H i survey, like our own, not only can identify faint, gas-rich galaxies but it also provides a measure of the dynamical mass of the galaxy through the Hi linewidth. Such surveys, however, are unlikely to detect the lowest mass galaxies, e.g., the dwarf spheroidals, that tend to lack detectable amounts of H I (Grcevich \& Putman 2009).

To construct a CVDF for the six loose groups in this study, we start with the measured $W_{20}$ from the integrated H I spectrum. Following the procedure of Meyer et al. (2008), we first correct $W_{20}$ for instrumental broadening using $W_{20, s}=W_{20}-0.55 R$, where $R$ is the spectral resolution (either 1.65 or $3.3 \mathrm{~km} \mathrm{~s}^{-1}$ for the Parkes data or in Table 4 for ATCA data). We then correct the linewidth for turbulent broadening following the prescription of Tully \& Fouque (1985):

$$
W_{R}^{2}=W_{20, s}^{2}-W_{t}^{2}-2 W_{20, s} W_{t}\left[1-e^{-\left(W_{20} / W_{c}\right)^{2}}\right]-2 W_{t}^{2} e^{-\left(W_{20} / W_{c}\right)^{2}}
$$

using $W_{c}$, the transition between single- and double-peaked profiles, of $120 \mathrm{~km} \mathrm{~s}^{-1}$, and $W_{t}$, the turbulence correction, of $22 \mathrm{~km} \mathrm{~s}^{-1}$ to obtain the full rotation amplitude, $W_{R}$. Finally, we apply an inclination correction to each linewidth and divide by two to get $V_{\text {rot }}$ as shown in Figure 35 . We assume that $V_{\text {rot }}$ is equal to $V_{\text {circ }}$ for the associated dark matter halos of all of our group galaxies (Klypin et al. 1999). Note that this approach assumes that all of the group galaxies are rotating and are not supported by random motions.

For each galaxy, we took the inclination from HyperLeda ${ }^{10}$ or used the same formula with the axial ratio from the

\footnotetext{
10 http://leda.univ-lyon1.fr/
}

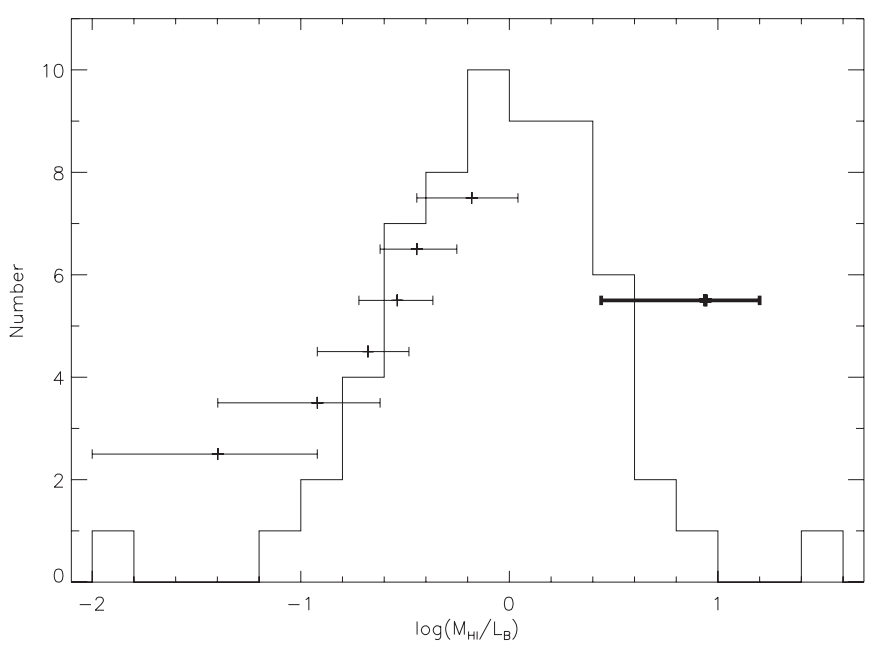

Figure 32. Histogram of the $M_{\mathrm{H}} / L_{B}$ ratio for our group galaxies. The error bars are as in Figure 31, but for the $M_{\mathrm{HI}} / L_{B}$ ratio. Local Group dwarf galaxy data are from Mateo (1998), Grcevich \& Putman (2009), and Kalirai et al. (2010). Approximately half of our group galaxies detected in $\mathrm{H}$ I are more gas-rich than even Magellanic spirals and irregulars (Roberts \& Haynes 1994). Again, our survey is primarily finding gas-rich dwarf galaxies.

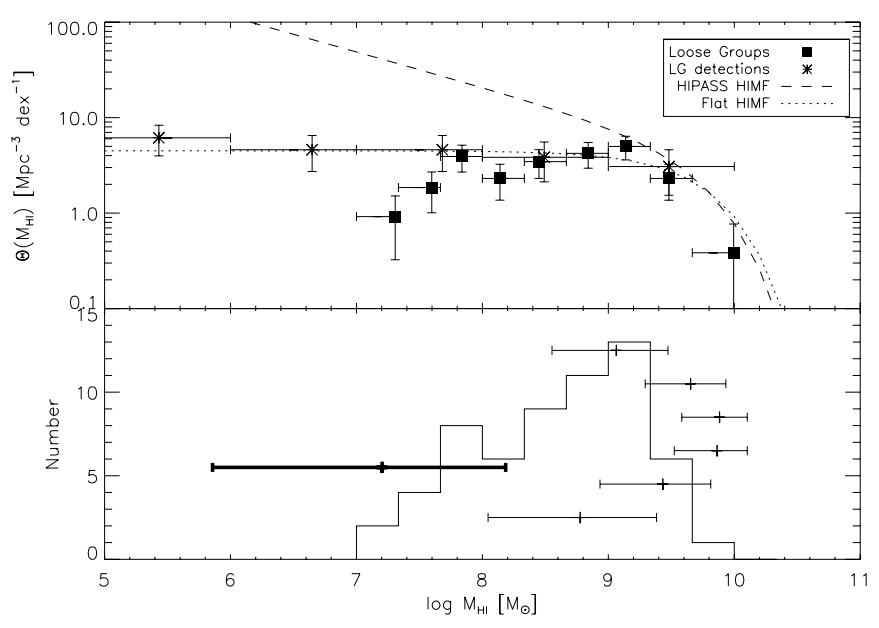

Figure 33. Top: the H I mass function for our sample of six loose groups (squares) as compared to the Local Group galaxies detect in $\mathrm{H}_{\mathrm{I}}$ (asterisks). $M_{\mathrm{H}_{\mathrm{I}}}$ for Local Group galaxies comes from Mateo (1998) and Grcevich \& Putman (2009). The points are plotted at the mean $M_{\mathrm{H}_{\mathrm{I}}}$ for the galaxies in each bin. The horizontal extent of the error bars represents the bin size, while their vertical extent represents the Poisson noise. The solid line represents a flat Schechter function $(\alpha=-1.0)$ roughly normalized to the Local Group. The dashed line is the HIPASS H I mass function from Zwaan et al. (2005) normalized to match our data points. Bottom: the raw number of galaxies in each $M_{\mathrm{H}_{\mathrm{I}}}$ bin for our loose groups. The error bars are as in Figure 31, but for $M_{\mathrm{H}_{\mathrm{I}}}$. Local Group dwarf galaxy data are from Mateo (1998) and Grcevich \& Putman (2009). It is clear from this comparison that most of the galaxies have $M_{\mathrm{H}_{\mathrm{I}}}$ consistent with a late-type spiral galaxy or a dwarf irregular galaxy.

NASA/IPAC Extragalactic Database (NED): ${ }^{11}$

$$
\sin ^{2} i=\frac{1-10^{-2 \log r_{25}}}{1-10^{-2 \log r_{o}}}
$$

where $r_{25}$ is the axial ratio and $\log r_{o}=0.38$, appropriate for galaxies with Hubble types later than Sd (Paturel et al. 2003). For one galaxy, APMUKS B1237-0648, this method yielded $\sin i>1$, so we chose $i=90^{\circ}$. Very few of our galaxies were observed with sufficiently high resolution to derive a kinematic

11 http://nedwww.ipac.caltech.edu/ 


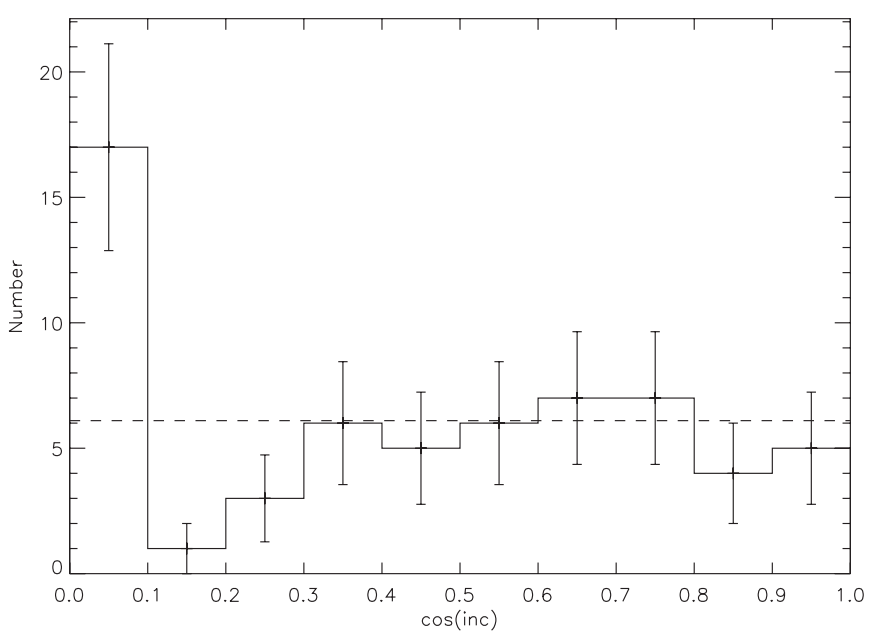

Figure 34. Distribution of group galaxy inclinations. If randomly selected, the cosine of the inclination should be a flat distribution. The distribution deviates from a random distribution at high inclinations.

inclination, but for those galaxies that have high quality, highresolution data, NGC 1249, UGCA 168, NGC 2997, IC 5332, and UGCA 320, the kinematic inclinations agree to within $10^{\circ}$ of the optical inclinations. In Figure 34, we plot the cosine of the inclination for all group galaxies. While the distribution is largely consistent with the disks being randomly distributed, there is a $2.6 \sigma$ excess of galaxies with $\cos i<0.1$, a $5 \sigma$ deficit of galaxies with $\cos i=0.1-0.2$, and a $1.8 \sigma$ deficit of galaxies with $\cos i=0.2-0.3$. If all those extra galaxies with $\cos i<0.1$ were redistributed to the other two bins, this would result in an increase of derived $V_{\text {rot }}$ values of only $5 \%$. Since the inclination also shows no correlation with either the integrated flux or the linewidth of the galaxy, we have applied no correction to the inclinations. To create the CVDF, the group galaxies were placed in bins of 0.3 dex width with a weighting based on the completeness of the survey as a function of linewidth and integrated H I flux. This results in the solid points as shown in Figure 35.

For comparison, we have also created a CVDF for the Local Group galaxies both detected and undetected in $\mathrm{H}$. The linewidth data for the Local Group galaxies comes from a variety of sources: for the Milky Way and M 31 the rotation velocity comes from van den Bergh (2000); M 33 from Corbelli \& Schneider (1997), the LMC from Kim et al. (1998); the SMC from Stanimirovic et al. (1999); and the rest of the dwarf galaxies from Mateo (1998), Longmore et al. (1982), Simon \& Geha (2007), Martin et al. (2007), Geha et al. (2009), Walker et al. (2009), and Kalirai et al. (2010). For some of these galaxies, there are measurements of their $V_{\text {rot }}$ from stars or H I. For those galaxies without measured rotation, or which have velocity dispersions greater than the rotation velocity, we assume isotropic orbits in an isothermal halo, so $V_{\text {rot }}=\sqrt{2} \sigma$. In Figure 35 we plot the CVDF for all the Local Group galaxies and, separately, only for those which have been detected in $\mathrm{H}$. As for the HIMF, we assume that the average survey volume per group is equal to the volume of the Local Group. Just like the HIMF, there is excellent agreement between the loose group CVDF and the Local Group CVDF for H I-rich galaxies, except for the lowest $V_{\text {rot }}$ bin, where the completeness of the group survey is more poorly estimated.

In addition to the Local Group, we compare the group CVDF with three other observed CVDFs and the results of simulations. As for the HIMF, these CVDFs have been renormalized to

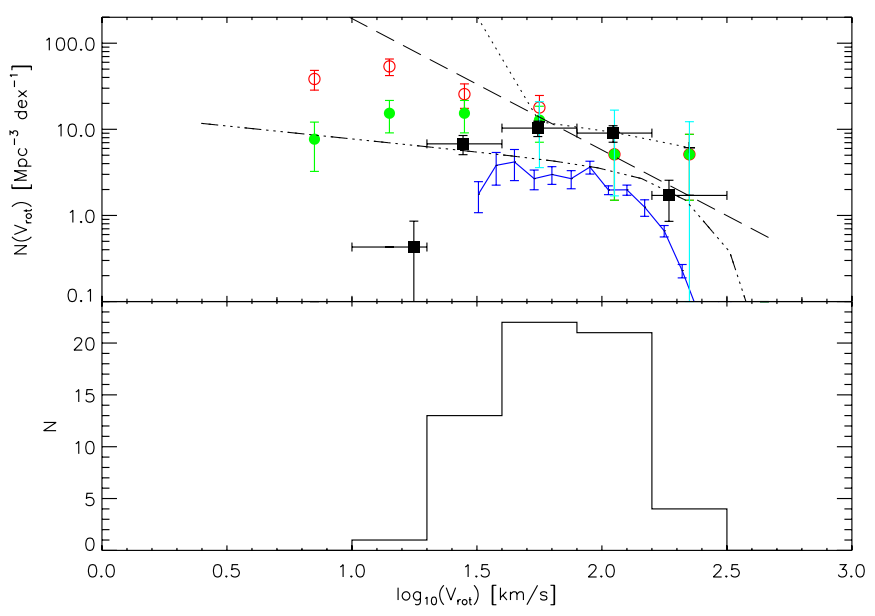

Figure 35. Top: the circular velocity distribution function (CVDF) for the Local Group (circles) and loose groups (squares). The loose group data are plotted at the mean $V_{\text {rot }}$ for each bin. The filled circles are the CVDF for the Local Group derived only for galaxies with $\mathrm{H}_{\mathrm{I}}$ detections. The open circles include Local Group galaxies that have dynamical data from stellar kinematics. The data for the Local Group data for dwarf galaxies come from Mateo (1998), Simon \& Geha (2007), Kalirai et al. (2010), Walker et al. (2009), and Geha et al. (2009). Data for the LMC come from Kim et al. (1998), the SMC Stanimirovic et al. (1999), M33 Corbelli \& Schneider (1997), while the Milky Way and M31 data are from van den Bergh (2000). The solid line with error bars is the CVDF for HIPASS detections from Zwaan et al. (2010). The dashed line represents the CVDF for cluster galaxies from Desai et al. (2004), while the dot-dash line is for field galaxies from Gonzalez et al. (2000). Finally, the dotted line with the cyan error bars is the CVDF construction from the Via Lactea II simulations (Diemand et al. 2008). All CVDFs aside from those for the loose groups and the Local Group have been renormalized to roughly match our data. Bottom: the raw number of galaxies in each $V_{\text {rot }}$ bin for our loose groups.

(A color version of this figure is available in the online journal.)

approximately match the group CVDF. The solid line with error bars is the HIPASS CVDF from Zwaan et al. (2010). The ALFALFA velocity width function (Papastergis et al. 2011) is almost identical to the HIPASS velocity width function (Zwaan et al. 2010). Note that Papastergis et al. (2011) did not convert the velocity widths into rotation velocities, so it is not directly comparable to the other functions here, but does extend down to half the velocity width of the Zwaan et al. (2010) results and has almost five times the number of sources. The dashed line is a cluster CVDF from Desai et al. (2004), while the dot-dash line is for field galaxies from Gonzalez et al. (2000). Aside from the normalization, the slopes of the CVDF at low $V_{\text {rot }}$ are in good agreement between the loose groups, HIPASS, and the Gonzalez et al. (2000) field galaxies. In contrast, there is a deficit of low-mass galaxies in loose groups as compared to the Desai et al. (2004) cluster sample. In addition, if we compare to the CVDF from the Via Lactea II results (Diemand et al. 2008), we see a deficit of low-mass galaxies in loose groups; this is the standard definition of the "missing satellite" problem. Note that the Via Lactea II simulation is a dark matter-only simulation of a Milky Way-sized halo and its sub-halos, so the resulting CVDF cannot be directly compared to larger halos. The loose groups and Local Group, however, are not significantly more massive than the parent halo in these simulations.

Examining the CVDF as a function of galaxy density from low-density groups through the field to the cluster environment, we do not see any significant differences except when we compare it with the highest density cluster environment. While Zwaan et al. (2010) did not look at the CVDF as a function of environment, they did examine the effects of cosmic variance 
and found that there were no significant differences in the slope of the CVDF between different quadrants of the sky. The standard way to explain the differences between the predictions of simulations and theory and the observed CVDF is the inclusion of the proper baryon physics. The clear difference between the CVDF in clusters and the CVDF of the field and groups provides an additional constraint on the possible explanations between observations and theory. Explanations of the difference between theory and observations include: dwarf galaxies inhabit only the most massive halos today (Stoehr et al. 2002) or only the most massive halos when they were accreted by a larger halo (Kravtsov et al. 2004), or only those that collapsed before reionization (Bullock et al. 2000, cf. Fenner et al. 2006). If, however, the discrepancy is due to the presence of warm dark matter instead of CDM, this should be independent of environment. In their study of the "missing satellite" problem, Simon \& Geha (2007) found the best match with their data for Local Group dwarfs if they only considered those dwarf galaxy halos that collapsed before reionization. If this explanation holds for our sample, then low-mass halos in clusters must have collapsed before those in groups, as would be expected for higher density regions with shorter dynamical times.

\section{CONCLUSIONS}

We have conducted an $\mathrm{H}$ I survey of the entire area of six loose groups that are analogous to the Local Group using the Parkes multibeam receiver and the ATCA. Our survey has two goals: (1) to compare the H I properties of loose groups to the Local Group and other groups and (2) to examine how the HIMF and CVDF of loose groups compare to those in the Local Group, other environments, and simulations.

We found the following.

1. Our survey found $61 \mathrm{HI}$-rich galaxies in the six groups down to $M_{\mathrm{HI}}$ of $9 \times 10^{6} M_{\odot}$, roughly doubling the number of group galaxies as determined by Garcia (1993). All of the HI-detected objects have properties consistent with gas-rich spiral, irregular, or dwarf irregular galaxies. The derived masses of the groups surveyed, including the new detections, are all within an order of magnitude of the Local Group. All the groups have similar radial extent and mean separation of the large galaxies.

2. The HIMF of these loose groups has a flat low- $M_{\mathrm{HI}}$ slope that agrees very well with the HIMF of the Local Group. Both are flatter than the HIMF of field galaxies from HIPASS (Zwaan et al. 2005) and are consistent with the idea that the HIMF flattens as the local galaxy density decreases.

3. The CVDF of loose groups agrees very well with that of the Local Group H I-detected galaxies, although it is lower than the CVDF for all Local Group galaxies. The loose group CVDF has the same low- $V_{\text {rot }}$ slope as was found in an optical study of field galaxies by Gonzalez et al. (2000) and for HIPASS galaxies by Zwaan et al. (2010). The loose group CVDF low- $V_{\text {rot }}$ slope is significantly flatter than that of cluster galaxies (Desai et al. 2004) or predicted by dark matter only simulations of Milky Way-sized halos (Diemand et al. 2008). Only in dense clusters are their significant differences in the CVDF from the field or groups.

4. Overall, our survey shows that the Local Group is not atypical in terms of the $\mathrm{H}$ I properties of its galaxies nor the properties of the dark matter halos hosting $\mathrm{H}$ I-rich galaxies.

While our survey has provided measurements of the HIMF and CVDF in the loose group environment, they are based on a relatively small number of galaxies, only 61 . The restricted range of group density and morphology probed by previous studies and the small number of group members have been the main limitations of past studies, including our own. Fortunately, currently ongoing and planned H I surveys will help improve this situation in a variety of ways. ALFALFA (Giovanelli et al. 2005) is detecting galaxies out to larger distances and down to lower $M_{\mathrm{H}}$ than HIPASS, but only $40 \%$ of the survey has been used for the published HIMF and CVDF (Martin et al. 2010; Papastergis et al. 2011). ALFALFA allows the study of relatively massive galaxy properties over a range of environments, however the Arecibo Galaxy Environment Survey (Auld et al. 2006) is studying a wide range of environments from isolated galaxies through galaxy groups to galaxy clusters down to lower $M_{\mathrm{HI}}$. In the future, planned H I surveys with Square Kilometer Array pathfinder instruments, such as WALLABY with ASKAP $^{12}$ or with APERTIF on the Westerbork Synthesis Radio Telescope (Oosterloo et al. 2010), will further improve our understanding of how H i properties of galaxies vary with environment.

The authors thank the staff at Parkes and the ATCA for their assistance with observing. We thank Warwick Wilson for his excellent work in making the $16 \mathrm{MHz}$ filters for these observations. The authors thank Martin Zwaan for his assistance inserting simulated sources into our data cubes and Karen Masters for providing the distances to our galaxy groups. We also thank the anonymous referee for his/her prompt review and helpful comments which improved this paper. This research was performed in part while D.J.P. held a National Research Council Research Associateship Award at the Naval Research Laboratory. D.J.P. also acknowledges generous support from NSF MPS International Distinguished Research Fellowship grant AST 0104439 and partial support from an ATNF Bolton Fellowship. B.K.G. acknowledges the generous financial support provided by Saint Mary University's Visitor Program. The authors also thank Bill Saxton for his assistance in making the group figures. We acknowledge the usage of the HyperLeda database (http://leda.univ-lyon1.fr). This research has made use of the NASA/IPAC Extragalactic Database (NED) which is operated by the Jet Propulsion Laboratory, California Institute of Technology, under contract with the National Aeronautics and Space Administration.

\section{REFERENCES}

Auld, R., Minchin, R. F., Davies, J. I., et al. 2006, MNRAS, 371, 1617

Blanton, M. R., Geha, M., \& West, A. A. 2008, ApJ, 682, 861

Borthakur, S., Yun, M. S., \& Verdes-Montenegro, L. 2010, ApJ, 710, 385

Bravo-Alfaro, H., Cayatte, V., van Gorkom, J. H., \& Balkowski, C. 2000, AJ, 119,580

Bravo-Alfaro, H., Cayatte, V., van Gorkom, J. H., \& Balkowski, C. 2001, A\&A, 379,347

Bullock, J. S., Kravtsov, A. V., \& Weinberg, D. H. 2000, ApJ, 539, 517

Cattaneo, A., Dekel, A., Devriendt, J., Guiderdoni, B., \& Blaizot, J. 2006, MNRAS, 370, 1651

Chung, A., van Gorkom, J. H., Kenney, J. D. P., Crowl, H., \& Vollmer, B. 2009, AJ, 138, 1741

Cole, S., \& Kaiser, N. 1989, MNRAS, 237, 1127

Corbelli, E., \& Schneider, S. E. 1997, ApJ, 479, 244

Croton, D. J., Farrar, G. R., Norberg, P., et al. 2005, MNRAS, 356, 1155

Davies, J., Minchin, R., Sabatini, S., et al. 2004, MNRAS, 349, 922

Desai, V., Dalcanton, J. J., Mayer, L., et al. 2004, MNRAS, 351, 265

Diemand, J., Kuhlen, M., Madau, P., et al. 2008, Nature, 454, 735

Duffy, A. R., Kay, S. T., Battye, R. A., et al. 2011, MNRAS, submitted (arXiv:1107.3720)

\footnotetext{
12 http://www.atnf.csiro.au/research/WALLABY
} 
Eke, V. R., Baugh, C. M., Cole, S., et al. 2004, MNRAS, 348, 866

Fenner, Y., Gibson, B. K., Gallino, R., \& Lugaro, M. 2006, ApJ, 646, 184

Freeland, E., Stilp, A., \& Wilcots, E. 2009, AJ, 138, 295

Freeland, E., \& Wilcots, E. 2011, ApJ, 738, 145

Garcia, A. M. 1993, A\&AS, 100, 47

Gavazzi, G., Boselli, A., van Driel, W., \& O’Neil, K. 2005, A\&A, 429, 439

Gavazzi, G., O’Neil, K., Boselli, A., \& van Driel, W. 2006, A\&A, 449, 929

Geha, M., Willman, B., Simon, J. D., et al. 2009, ApJ, 692, 1464

Geller, M. J., \& Huchra, J. P. 1983, ApJS, 52, 61

Giovanelli, R., Haynes, M. P., Kent, B. R., et al. 2005, AJ, 130, 2598

Goldberg, D. M., Jones, T. D., Hoyle, F., et al. 2005, ApJ, 621, 643

Gonzalez, A. H., Williams, K. A., Bullock, J. S., Kolatt, T. S., \& Primack, J. R. 2000, ApJ, 528, 145

Grcevich, J., \& Putman, M. E. 2009, ApJ, 696, 385

Haynes, M. P., \& Giovanelli, R. 1984, AJ, 89, 758

Heisler, J., Tremaine, S., \& Bahcall, J. N. 1985, ApJ, 298, 8

Helsdon, S. F., \& Ponman, T. J. 2003, MNRAS, 339, L29

Hess, K. M., Pisano, D. J., Wilcots, E. M., \& Chengalur, J. N. 2009, ApJ, 699, 76

Hickson, P. 1982, ApJ, 255, 382

Hickson, P. 1997, ARA\&A, 35, 357

Hopkins, P. F., Somerville, R. S., Hernquist, L., et al. 2006, ApJ, 652, 864

Kalirai, J. S., Beaton, R. L., Geha, M. C., et al. 2010, ApJ, 711, 671

Kilborn, V. A., Forbes, D. A., Barnes, D. G., et al. 2009, MNRAS, 400, 1962

Kim, S., Staveley-Smith, L., Dopita, M. A., et al. 1998, ApJ, 503, 674

Klypin, A., Kravtsov, A. V., Valenzuela, O., \& Prada, F. 1999, ApJ, 522, 82

Kovač, K., Oosterloo, T. A., \& van der Hulst, J. M. 2005, in IAU Colloq. 198, Near-fields Cosmology with Dwarf Elliptical Galaxies, ed. H. Jerjen \& B. Binggeli (Cambridge: Cambridge Univ. Press), 351

Kovač, K., Oosterloo, T. A., \& van der Hulst, J. M. 2009, MNRAS, 400, 743

Kravtsov, A. V., Gnedin, O. Y., \& Klypin, A. A. 2004, ApJ, 609, 482

Longmore, A. J., Hawarden, T. G., Goss, W. M., Mebold, U., \& Webster, B. L. 1982, MNRAS, 200, 325

Martin, A. M., Papastergis, E., Giovanelli, R., et al. 2010, ApJ, 723, 1359

Martin, N. F., Ibata, R. A., Chapman, S. C., Irwin, M., \& Lewis, G. F. 2007, MNRAS, 380, 281

Masters, K. L. 2005, PhD thesis, Cornell Univ.

Mateo, M. L. 1998, ARA\&A, 36, 435

Meyer, M. J., Zwaan, M. A., Webster, R. L., Schneider, S., \& Staveley-Smith, L. 2008, MNRAS, 391, 1712

Meyer, M. J., Zwaan, M. A., Webster, R. L., et al. 2004, MNRAS, 350, 1195

Mo, H. J., Yang, X., van den Bosch, F. C., \& Jing, Y. P. 2004, MNRAS, 349, 205

Moore, B., Ghigna, S., Governato, F., et al. 1999, ApJ, 524, L19

Mulchaey, J. S. 2000, ARA\&A, 38, 289

Mulchaey, J. S., Davis, D. S., Mushotzky, R. F., \& Burstein, D. 2003, ApJS, 145,39

Omar, A., \& Dwarakanath, K. S. 2005, JA\&A, 26, 71

Oosterloo, T., Verheijen, M., \& van Cappellen, W. 2010, in Proc. ISKAF2010 Science Meeting (Trieste: Proc. of Sci.), 43

Papastergis, E., Martin, A. M., Giovanelli, R., \& Haynes, M. P. 2011, ApJ, 739, 38
Paturel, G., Petit, C., Prugniel, P., et al. 2003, A\&A, 412, 45

Peek, J. E. G. 2009, ApJ, 698, 1429

Pisano, D. J., Barnes, D. G., Gibson, B. K., et al. 2004, ApJ, 610, L17

Pisano, D. J., Barnes, D. G., Gibson, B. K., et al. 2007, ApJ, 662, 959 (Paper I)

Pisano, D. J., \& Wilcots, E. M. 1999, AJ, 117, 2168

Pisano, D. J., Wilcots, E. M., \& Liu, C. T. 2002, ApJS, 142, 161

Ponman, T. J., Bourner, P. D. J., Ebeling, H., \& Böhringer, H. 1996, MNRAS, 283,690

Postman, M., \& Geller, M. J. 1984, ApJ, 281, 95

Roberts, M. S., \& Haynes, M. P. 1994, ARA\&A, 32, 115

Sandage, A. 1986, ApJ, 307, 1

Schlegel, D. J., Finkbeiner, D. P., \& Davis, M. 1998, ApJ, 500, 525

Sengupta, C., \& Balasubramanyam, R. 2006, MNRAS, 369, 360

Sengupta, C., Balasubramanyam, R., \& Dwarakanath, K. S. 2007, MNRAS, 378,137

Sheth, R. K., Bernardi, M., Schechter, P. L., et al. 2003, ApJ, 594, 225

Shimasaku, K. 1993, ApJ, 413, 59

Simon, J. D., \& Geha, M. 2007, ApJ, 670, 313

Snaith, O. N., Gibson, B. K., Brook, C. B., et al. 2011, MNRAS, 415, 2798

Spergel, D. N., Verde, L., Peiris, H. V., et al. 2003, ApJS, 148, 175

Springob, C. M., Haynes, M. P., \& Giovanelli, R. 2005, ApJ, 621, 215

Stanimirovic, S., Staveley-Smith, L., Dickey, J. M., Sault, R. J., \& Snowden, S. L. 1999, MNRAS, 302, 417

Staveley-Smith, L., Kim, S., Calabretta, M. R., Haynes, R. F., \& Kesteven, M. J. 2003, MNRAS, 339, 87

Staveley-Smith, L., Wilson, W. E., Bird, T. S., et al. 1996, PASA, 13, 243

Stevens, J. B. 2005, PhD thesis, Univ. Melbourne

Stierwalt, S., Haynes, M. P., Giovanelli, R., et al. 2009, AJ, 138, 338

Stoehr, F., White, S. D. M., Tormen, G., \& Springel, V. 2002, MNRAS, 335, L84

Tago, E., Einasto, J., Saar, E., et al. 2008, A\&A, 479, 927

Trentham, N., Sampson, L., \& Banerji, M. 2005, MNRAS, 357, 783

Tully, R. B. 1987, ApJ, 321, 280

Tully, R. B., \& Fouque, P. 1985, ApJS, 58, 67

Tully, R. B., Somerville, R. S., Trentham, N., \& Verheijen, M. A. W. 2002, ApJ, 569,573

van den Bergh, S. (ed.) 2000, The Galaxies of the Local Group (Cambridge Astrophysics Series, Vol. 35; Cambridge: Cambridge Univ. Press)

Verdes-Montenegro, L., Yun, M. S., Williams, B. A., et al. 2001, A\&A, 377, 812

Verheijen, M. A. W., Trentham, N., Tully, B., \& Zwaan, M. 2001, in ASP Conf Proc. Ser. 240, Gas and Galaxy Evolution, ed. J. E. Hibbard, M. Rupen, \& J. H. van Gorkom (San Francisco, CA: ASP), 507

Wakker, B. P., \& van Woerden, H. 1997, ARA\&A, 35, 217

Walker, M. G., Belokurov, V., Evans, N. W., et al. 2009, ApJ, 694, L144

Williams, B. A., McMahon, P. M., \& van Gorkom, J. H. 1991, AJ, 101, 1957

Williams, B. A., \& Rood, H. J. 1987, ApJS, 63, 265

Zabludoff, A. I., \& Mulchaey, J. S. 1998, ApJ, 498, L5

Zwaan, M. A., Meyer, M. J., \& Staveley-Smith, L. 2010, MNRAS, 403, 1969

Zwaan, M. A., Meyer, M. J., Staveley-Smith, L., \& Webster, R. L. 2005, MNRAS, 359, L30

Zwaan, M. A., Meyer, M. J., Webster, R. L., et al. 2004, MNRAS, 350, 1210

Zwaan, M. A., Staveley-Smith, L., Koribalski, B. S., et al. 2003, AJ, 125, 2842 


\title{
ERRATUM: “AN H I SURVEY OF SIX LOCAL GROUP ANALOGS. II. H I PROPERTIES OF GROUP GALAXIES” (2011, ApJS, 197, 28)
}

\author{
D. J. Pisano ${ }^{1,7}$, David G. Barnes ${ }^{2}$, Lister Staveley-Smith ${ }^{3}$, \\ Brad K. Gibson 4,5 , Virginia A. Kilborn ${ }^{2}$, and Ken C. Freeman ${ }^{6}$ \\ ${ }^{1}$ Department of Physics, West Virginia University, P.O. Box 6315, Morgantown, WV, 26506, USA; djpisano@mail.wvu.edu \\ ${ }^{2}$ Centre for Astrophysics \& Supercomputing, Swinburne University, Hawthorn, Victoria 3122, Australia; David.G.Barnes@gmail.com, vkilborn@astro.swin.edu.au \\ ${ }^{3}$ International Centre for Radio Astronomy Research, M468, University of Western Australia, Crawley, WA 6009, Australia; Lister.Staveley-Smith @icrar.org \\ ${ }^{4}$ Jeremiah Horrocks Institute, University of Central Lancashire, Preston PR1 2HE, UK \\ ${ }^{5}$ Department of Astronomy \& Physics, Saint Mary's University, Halifax, Nova Scotia B3H 3C3, Canada; brad.k.gibson@ gmail.com \\ ${ }^{6}$ RSAA, Mount Stromlo Observatory, Cotter Road, Weston, ACT 2611, Australia; kcf@ mso.anu.edu.au \\ Received 2012 July 3; published 2012 August 6
}

Online-only material: color figure

In Table 1 of the published article, the coordinates listed for UGCA 320 were given as B1950 instead of J2000. The correct J2000 coordinates from the $\mathrm{H}$ I data are a right ascension of 13:03:17.3 and a declination of -17:25:21. All other parameters for this galaxy are correct, but this correction affects the derived group properties in Table 3. For the HIPASS Group, the group diameter is now $0.82 \mathrm{Mpc}$, the mean galaxy-galaxy separation is $0.57 \mathrm{Mpc}$, and the radial dispersion is $0.44 \mathrm{Mpc}$. The velocity of the group and the velocity dispersion of the group remain unchanged. The group virial mass changes to $0.4 \pm 0.2 \times 10^{12} M_{\odot}$, with a corresponding zero-velocity radius of $0.66 \mathrm{Mpc}$. The projected mass estimator yields a mass of $12.6 \pm 6.3 \times 10^{12} M_{\odot}$, with a zero-velocity radius of $2.1 \mathrm{Mpc}$. No conclusions of the paper are affected by these changes.

When measuring the Hi properties of ESO 373-G7 and UGCA 168, their properties, as measured from the Parkes data, were confused. We present the new combined Parkes Hi spectrum (replacing the panel in Figure 4) below. We have remeasured the Hi properties from the ATCA data, scaling the Parkes Hi flux by the ratio of ATCA fluxes, and find that ESO 373-G7 has $V_{\odot}=862 \pm 1 \mathrm{~km} \mathrm{~s}^{-1}, W_{20}=54 \pm 2 \mathrm{~km} \mathrm{~s}^{-1}$, and $S_{\text {int }}=1.1 \pm 0.2 \mathrm{Jy} \mathrm{km} \mathrm{s}^{-1}$. For UGCA 168, we measure $V_{\odot}=926 \pm 2 \mathrm{~km} \mathrm{~s}^{-1}$, $W_{20}=226 \pm 4 \mathrm{~km} \mathrm{~s}^{-1}$, and $S_{\text {int }}=60.7 \pm 0.2 \mathrm{Jy} \mathrm{km} \mathrm{s}^{-1}$ (these values should replace those listed in Table 1 of the published article). This has also affected some of the derived properties of the LGG 180 group (Table 3). The group now has $V_{\odot}=1058 \mathrm{~km} \mathrm{~s}^{-1}$ and

ESO $373-7+$ UGCA 168

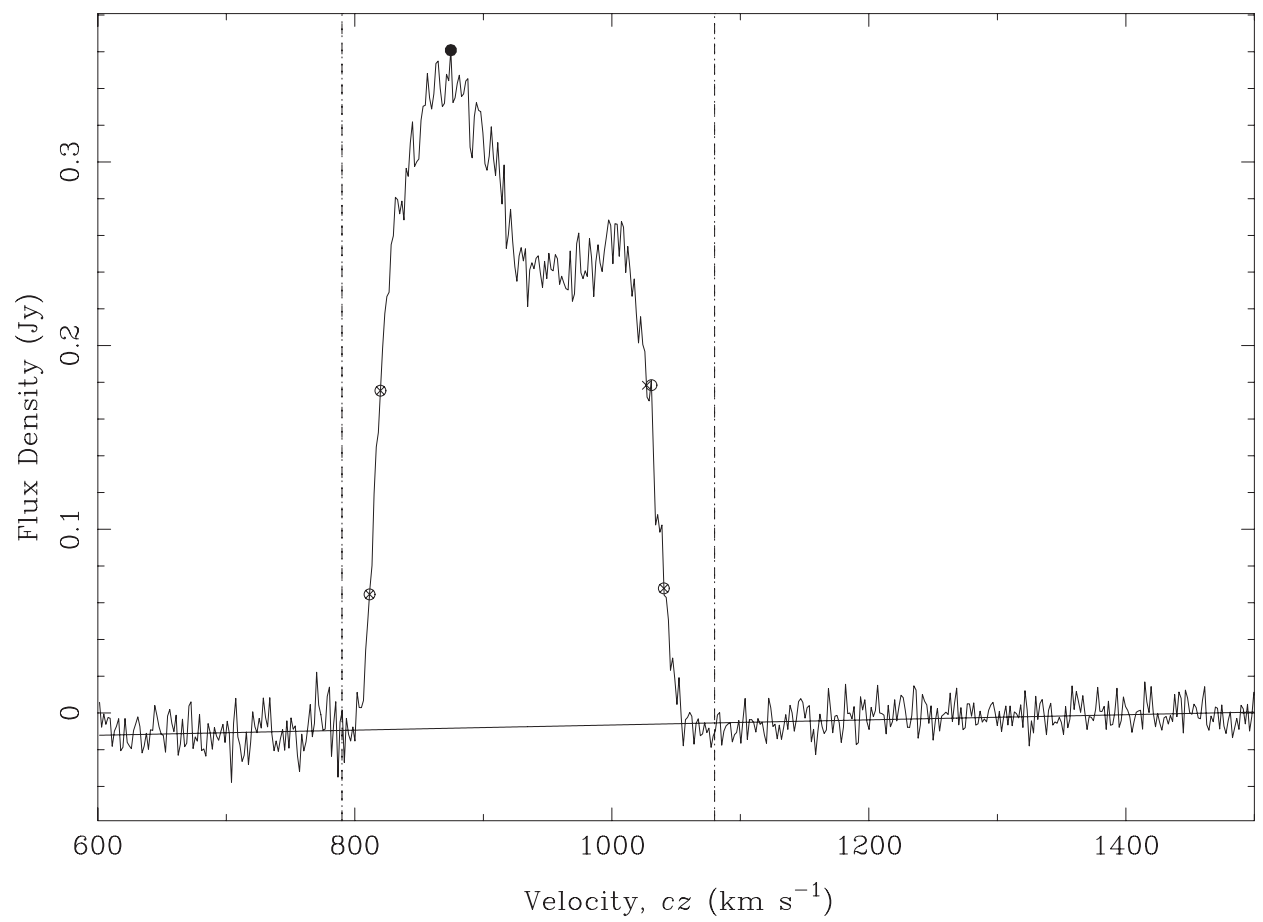

Figure 4. Combined Parkes H I spectrum of UGCA 168 and ESO 373-G7. The vertical dashed lines indicate the range of velocities over which the profile properties were measured. The filled circles indicate the peak of the profile; the open circles indicate the maximum $20 \%$ and $50 \%$ velocity widths; the $\times$ 's mark the minimum $20 \%$ and $50 \%$ widths. The nearly horizontal solid line indicates the baseline fit to the spectrum. This panel supersedes the individual panels for these galaxies in Figure 4 of the published article.

\footnotetext{
7 Adjunct Assistant Astronomer at National Radio Astronomy Observatory, P.O. Box 2, Green Bank, WV 24944, USA.
} 


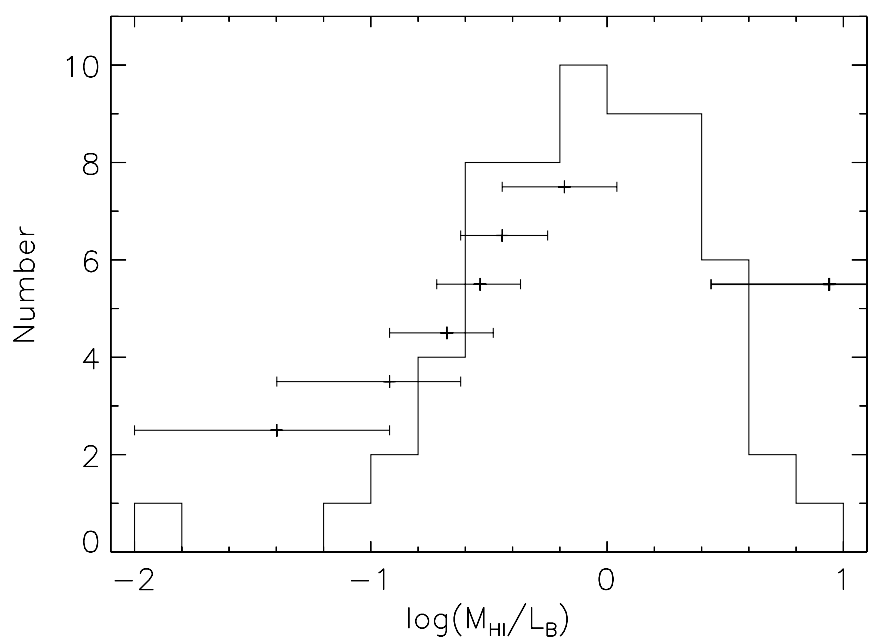

Figure 32. New histogram of the $M_{\mathrm{H}} / L_{B}$ ratio for our group galaxies. The error bars indicate the 25th, 50th, and 75th percentile distribution of $M_{B}$ for UGC galaxies as reported by Roberts \& Haynes (1994) and corrected for $H_{0}=72 \mathrm{~km} \mathrm{~s}^{-1} \mathrm{Mpc}^{-1}$. From bottom to top they represent these values for E/S0, S0a/Sa, Sab/Sb, Sbc/Sc, $\mathrm{Scd} / \mathrm{Sd}$, and Sm/Im galaxies. The thick error bar indicates the same thing for Local Group dwarf galaxies with H I detections from Mateo (1998) and Kalirai et al. (2010). Approximately half of our group galaxies detected in H I are more gas-rich than even Magellanic spirals and irregulars (Roberts \& Haynes 1994). Again, our survey is primarily finding gas-rich dwarf galaxies.

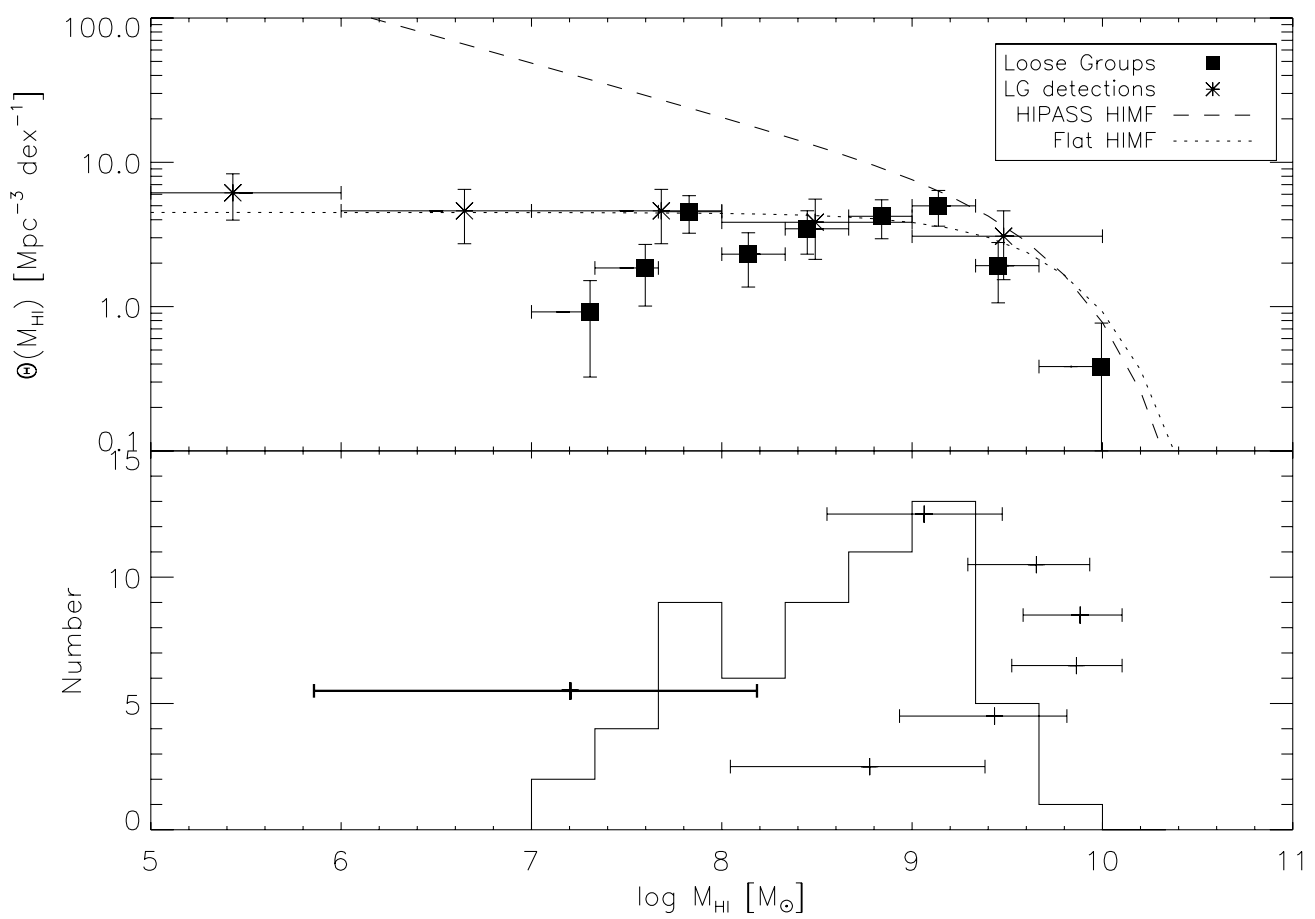

Figure 33. Top: the $\mathrm{H}$ I mass function for our sample of six loose groups (squares) as compared to the Local Group galaxies detected in $\mathrm{H}_{\mathrm{I}}$ (asterisks). $M_{\mathrm{H}}$ for Local Group galaxies comes from Mateo (1998) and Grcevich \& Putman (2009). The points are plotted at the mean $M_{\mathrm{H}_{\mathrm{I}}}$ for the galaxies in each bin. The horizontal extent of the error bars represents the bin size, while their vertical extent represents the Poisson noise. The solid line represents a flat Schechter function $(\alpha=-1.0)$ roughly normalized to the Local Group. The dashed line is the HIPASS H i mass function from Zwaan et al. (2005) normalized to match our data points. Bottom: the raw number of galaxies in each $M_{\mathrm{H}_{\mathrm{I}}}$ bin for our loose groups. The error bars are as in Figure 32, but for $M_{\mathrm{H}_{\mathrm{I}}}$. Local Group dwarf galaxy data are from Mateo (1998) and Grcevich \& Putman (2009). It is clear from this comparison that most of the galaxies have $M_{\mathrm{H}_{\mathrm{I}}}$ consistent with a late-type spiral galaxy or a dwarf irregular galaxy.

$\sigma_{v}=131 \mathrm{~km} \mathrm{~s}^{-1}$, while its virial mass is $7.9 \pm 2.5 \times 10^{12} M_{\odot}$ with a corresponding zero-velocity radius of $1.8 \mathrm{Mpc}$. The projected mass estimator yields a mass of $13 \pm 4 \times 10^{12} M_{\odot}$, with a corresponding zero-velocity radius of $2.1 \mathrm{Mpc}$. In Table $6, M_{\mathrm{H}}$ for ESO 373-G7 and UGCA 168 are now $0.6 \pm 0.1 \times 10^{8} M_{\odot}$ and $31.4 \pm 0.1 \times 10^{8} M_{\odot}$, respectively. Their $M_{\mathrm{HI}} / L_{B}$ are now 0.38 and 0.65 in solar units; this reduces the mass-to-light ratio of ESO 373-G7 from the highest in our sample to a value slightly below the median. Finally, their $V_{\text {rot }}$ are $16 \mathrm{~km} \mathrm{~s}^{-1}$ and $102 \mathrm{~km} \mathrm{~s}^{-1}$ for ESO 373-G7 and UGCA 168. These changes have no effect on the conclusions of the paper, but do make subtle changes to Figures 32, 33, and 35; the new versions are included here. 


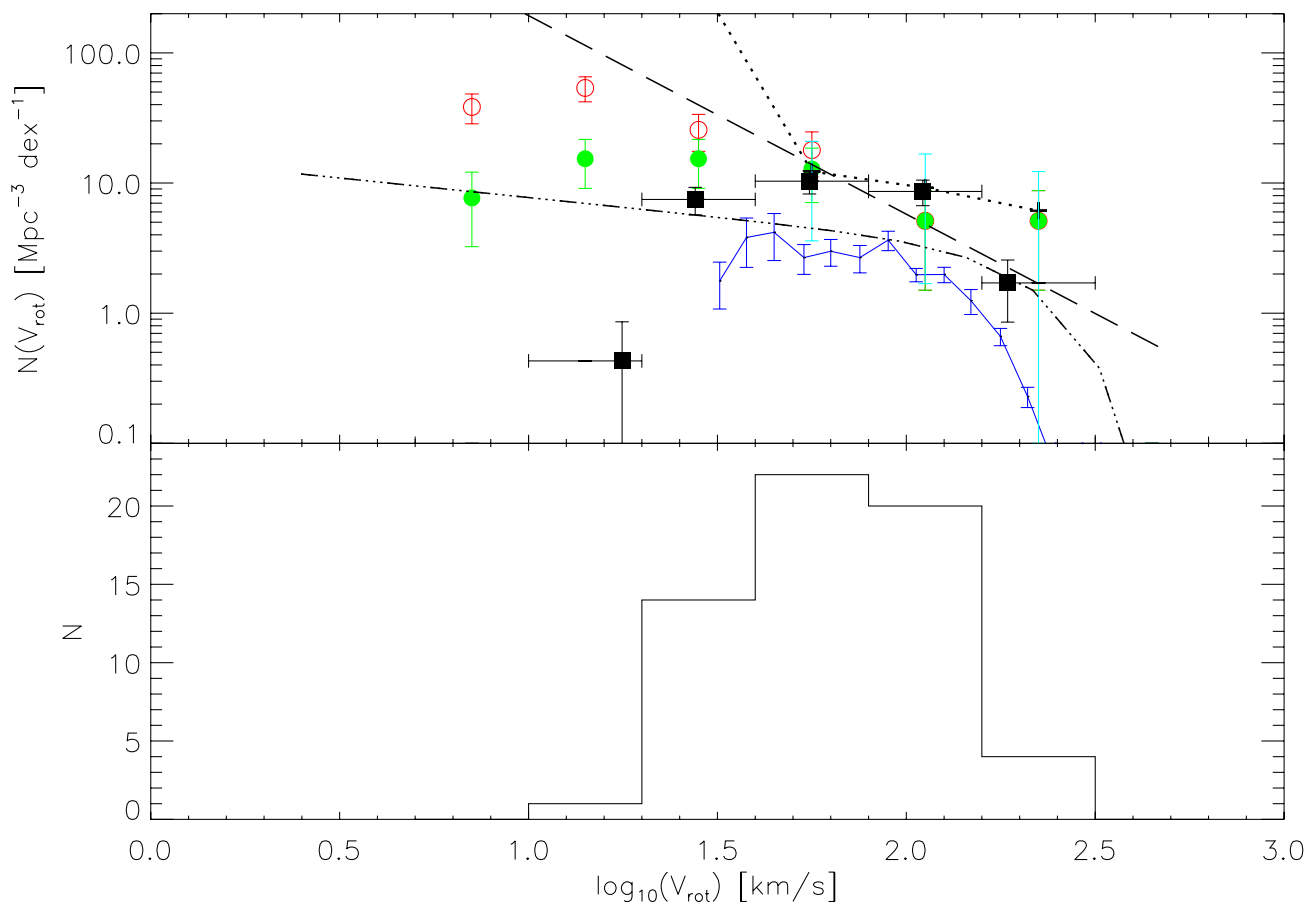

Figure 35. Top: the circular velocity distribution function (CVDF) for the Local Group (circles) and loose groups (squares). The loose group data are plotted at the mean $V_{\text {rot }}$ for each bin. The filled circles are the CVDF for the Local Group derived only for galaxies with $\mathrm{H}$ I detections. The open circles include Local Group galaxies that have dynamical data from stellar kinematics. The data for the Local Group data for dwarf galaxies come from Mateo (1998), Simon \& Geha (2007), Kalirai et al. (2010), Walker et al. (2009), and Geha et al. (2009). Data for the LMC come from Kim et al. (1998), the SMC from Stanimirovic et al. (1999), M33 from Corbelli \& Schneider (1997), while the Milky Way and M31 data are from van den Bergh (2000). The solid line with error bars is the CVDF for HIPASS detections from Zwaan et al. (2010). The dashed line represents the CVDF for cluster galaxies from Desai et al. (2004), while the dot-dashed line is for field galaxies from Gonzalez et al. (2000). Finally, the dotted line with the cyan error bars is the CVDF construction from the Via Lactea II simulations (Diemand et al. 2008). All CVDFs aside from those for the loose groups and the Local Group have been renormalized to roughly match our data. Bottom: the raw number of galaxies in each $V_{\text {rot }}$ bin for our loose groups.

(A color version of this figure is available in the online journal.)

Thanks to Igor Karachentsev for bringing these errors to our attention.

\section{REFERENCES}

Corbelli, E., \& Schneider, S. E. 1997, ApJ, 479, 244

Desai, V., Dalcanton, J. J., Mayer, L., et al. 2004, MNRAS, 351, 265

Diemand, J., Kuhlen, M., Madau, P., et al. 2008, Nature, 454, 735

Geha, M., Willman, B., Simon, J. D., et al. 2009, ApJ, 692, 1464

Gonzalez, A. H., Williams, K. A., Bullock, J. S., Kolatt, T. S., \& Primack, J. R. 2000, ApJ, 528, 145

Grcevich, J., \& Putman, M. E. 2009, ApJ, 696, 385

Kalirai, J. S., Beaton, R. L., Geha, M. C., et al. 2010, ApJ, 711, 671

Kim, S., Staveley-Smith, L., Dopita, M. A., et al. 1998, ApJ, 503, 674

Mateo, M. L. 1998, ARA\&A, 36, 435

Roberts, M. S., \& Haynes, M. P. 1994, ARA\&A, 32, 115

Simon, J. D., \& Geha, M. 2007, ApJ, 670, 313

Stanimirovic, S., Staveley-Smith, L., Dickey, J. M., Sault, R. J., \& Snowden, S. L. 1999, MNRAS, 302, 417

van den Bergh, S., ed. 2000, The Galaxies of the Local Group (Cambridge Astrophysics Series), Vol. 35 (Cambridge: Cambridge Univ. Press)

Walker, M. G., Belokurov, V., Evans, N. W., et al. 2009, ApJ, 694, L144

Zwaan, M. A., Meyer, M. J., \& Staveley-Smith, L. 2010, MNRAS, 403, 1969

Zwaan, M. A., Meyer, M. J., Staveley-Smith, L., \& Webster, R. L. 2005, MNRAS, 359, L30 